Aus der Medizinischen Klinik II

Bronchial- und Lungenheilkunde

(Chefarzt: Prof. Dr. med. G. Laier-Groeneveld)

des Klinikum Niederrhein in Oberhausen

\title{
Übertragungen von Keimen auf einer speziellen, vorwiegend nicht-invasiven Beatmungsintensivstation
}

\author{
INAUGURAL-DISSERTATION \\ zur Erlangung des Doktorgrades \\ der Medizinischen Fakultät der \\ Georg-August-Universität zu Göttingen
}

vorgelegt von

Lisa Barbara Hoffmann

aus

Mainz 
Dekan:

Referent:

Ko-Referent:

Drittreferentin:
Prof. Dr. rer. nat. H.K. Kroemer

Prof. Dr. Gerhard Laier-Groeneveld

PD Dr. Thorsten Perl

Prof. Dr. Simone Scheithauer

Datum der mündlichen Prüfung: Mittwoch, den 29.03.2017 
Hiermit erkläre ich, die Dissertation mit dem Titel "Übertragungen von Keimen auf einer speziellen vorwiegend nicht-invasiven Beatmungsintensivstation" eigenständig angefertigt und keine anderen als die von mir angegebenen Quellen und Hilfsmittel verwendet zu haben.

Göttingen, den 01.03.2017

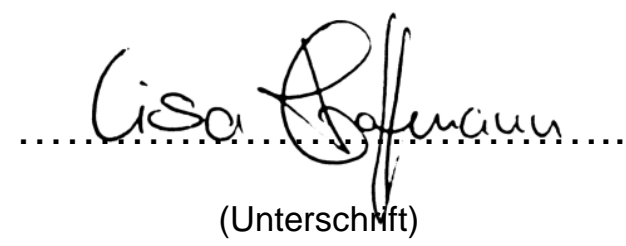




\section{Inhaltsverzeichnis}

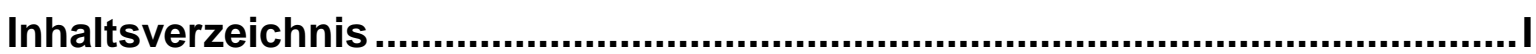

Abkürzungsverzeichnis ............................................................................. IV

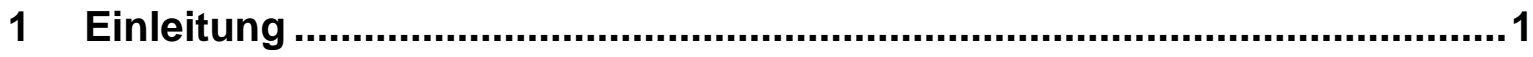

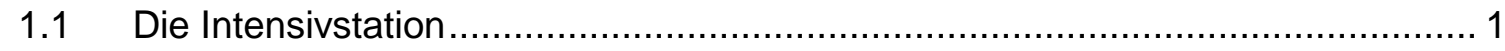

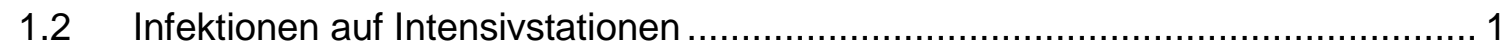

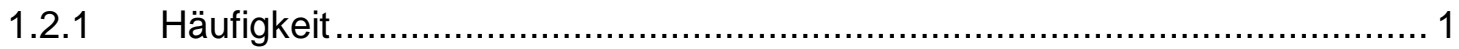

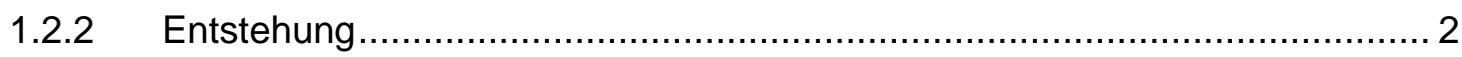

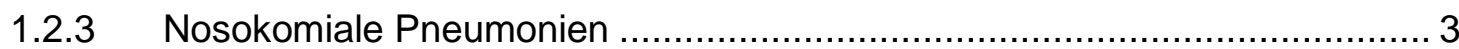

1.2.4 Präventionsmaßnahmen .................................................................. 4

1.3 Die „Beatmungsintensivstation“......................................................................

1.3.1 Die nicht-invasive Beatmung .......................................................... 5

1.3.2 Unterschiede gegenüber invasiver Beatmung ……………………….... 6

1.4 Die praktische Umsetzung einer „Beatmungsintensivstation“ im Klinikum

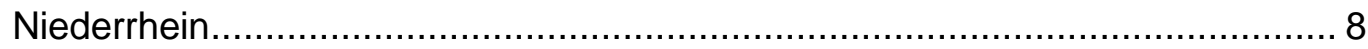

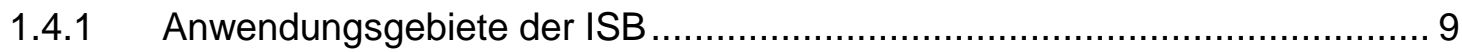

1.4.2 Praktische Umsetzung der Beatmungstherapie......................................... 11

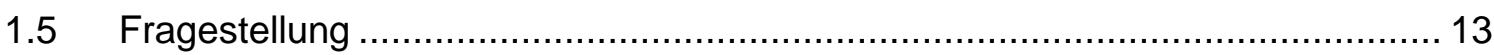

2 Material und Methoden ............................................................................ 14

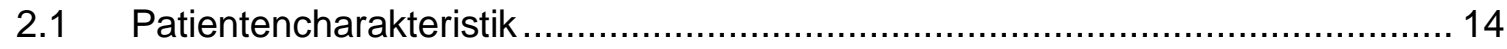

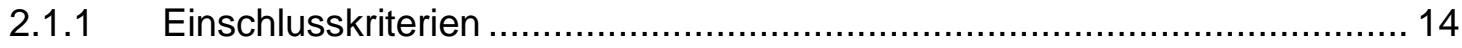

2.1.2 Grunderkrankungen und Ursache der Beatmung ................................... 14

2.1.3 Liegedauer, Anzahl und Dauer der Patientenkontakte ............................. 15

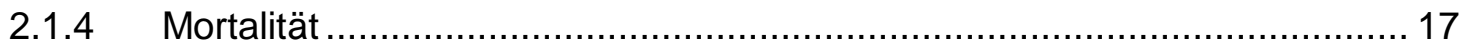

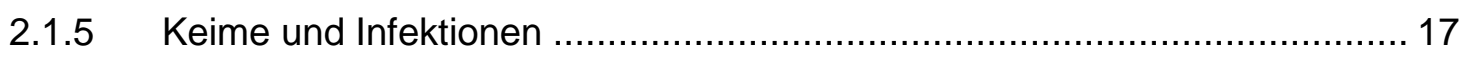

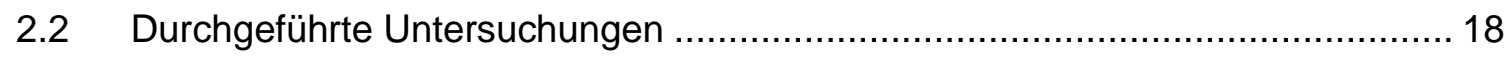

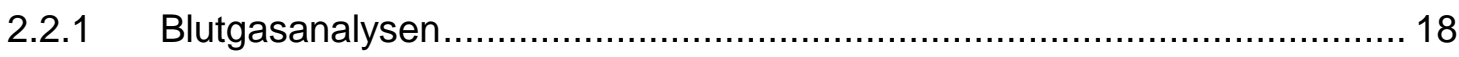

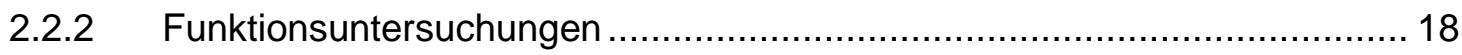

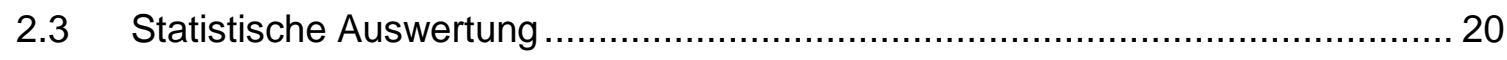

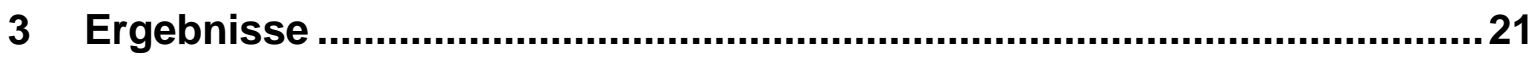


3.1 Grunderkrankungen und Ursachen der Beatmung .................................... 21

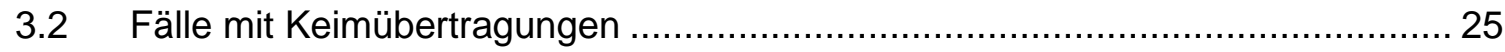

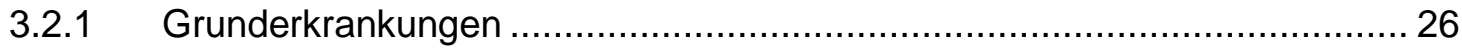

3.2.2 Ursache des Beatmungsbeginns.................................................. 27

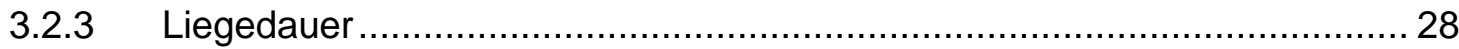

3.2.4 Anzahl der Patientenkontakte und gemeinsame Liegedauer der

Patientenkontakte............................................................................... 29

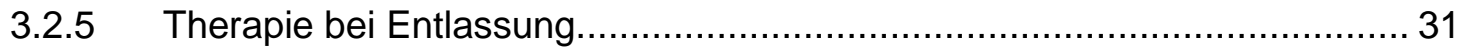

3.2.6 Mortalität und aufgetretene Infekte ...................................................... 31

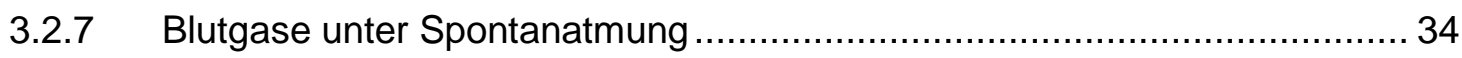

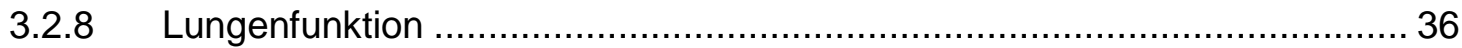

3.3 Übertragene Bakterienstämme............................................................. 38

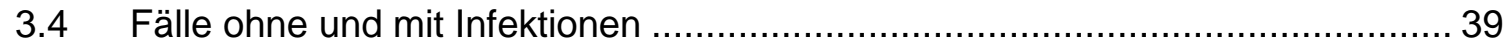

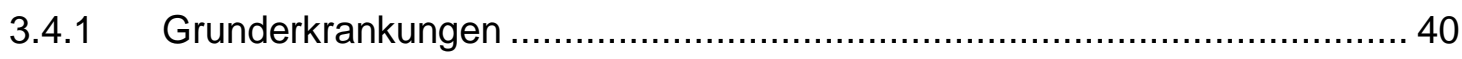

3.4.2 Ursache des Beatmungsbeginns .................................................. 41

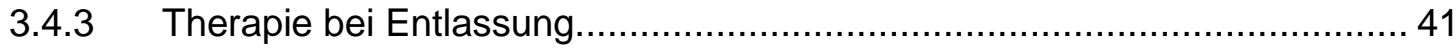

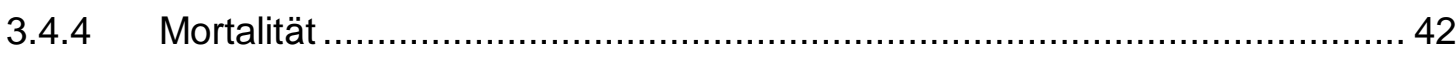

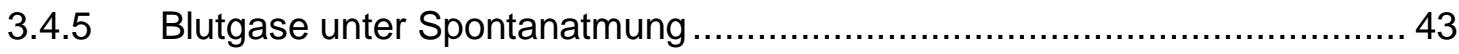

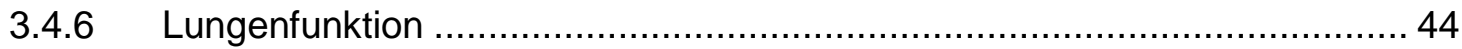

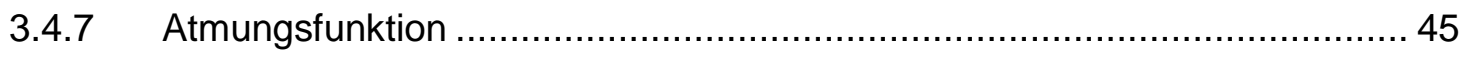

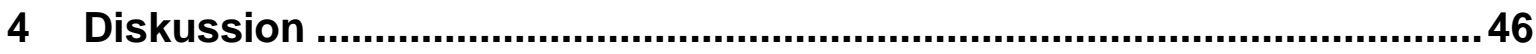

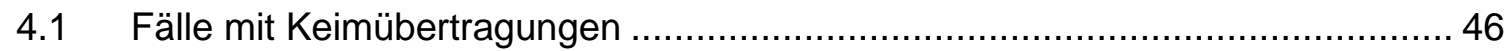

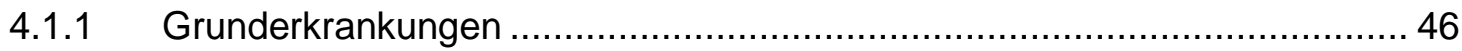

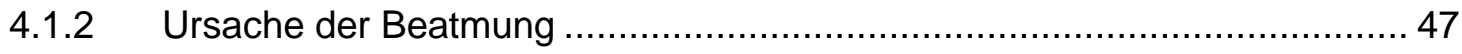

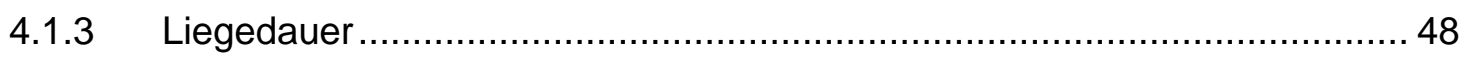

4.1.4 Anzahl der Patientenkontakte und gemeinsame Liegedauer der Patienten 48

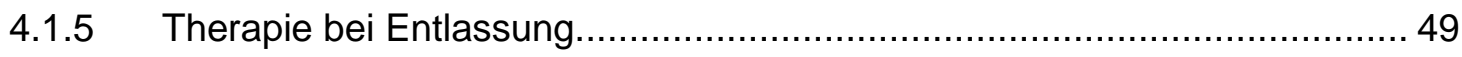

4.1.6 Mortalität und aufgetretene Infekte ................................................ 50

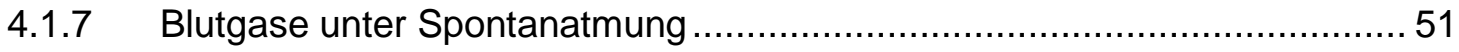

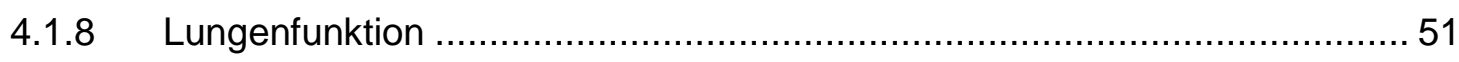




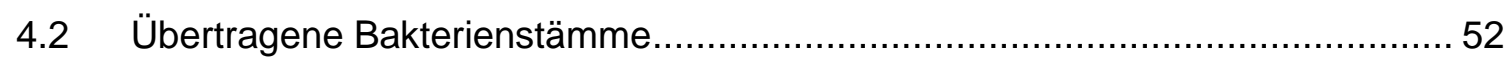

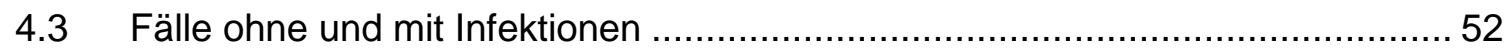

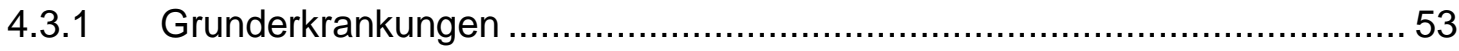

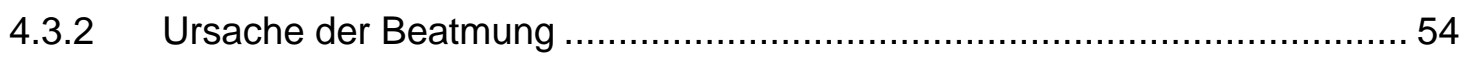

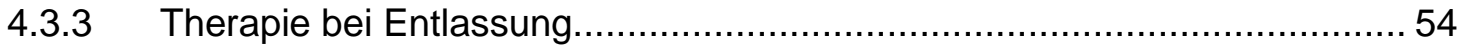

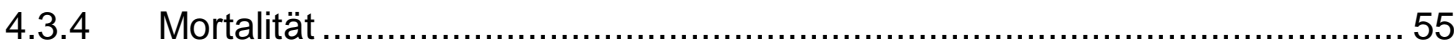

4.3.5 Blutgase unter Spontanatmung …................................................ 55

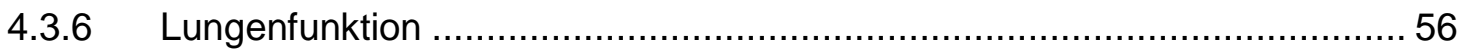

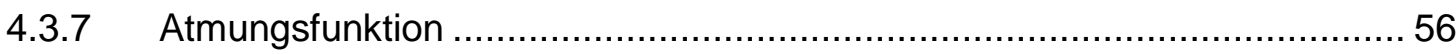

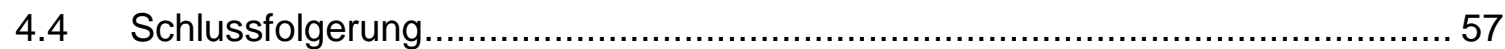

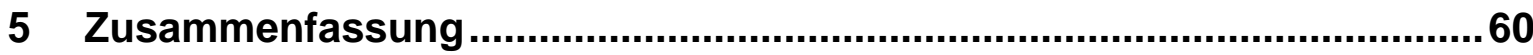

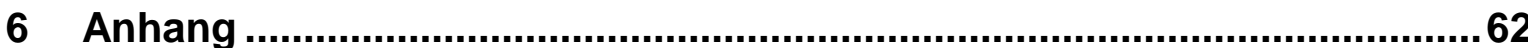

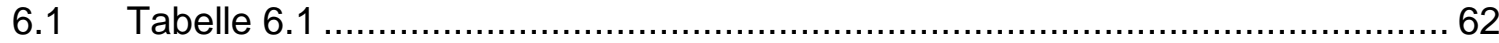

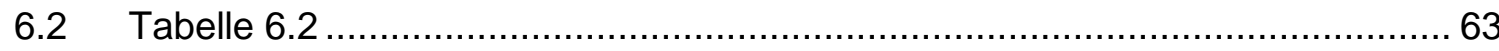

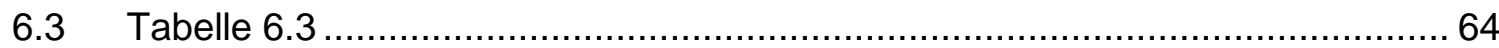

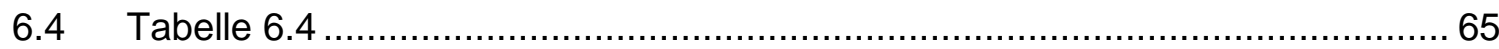

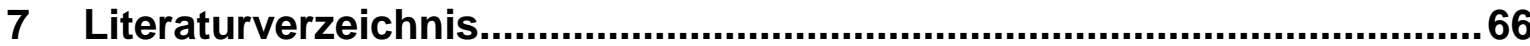




\section{Abkürzungsverzeichnis}

$\alpha$

ALS

ARI

BGA

COPD

$\mathrm{CO}_{2}$

DIGAB

E. coli

EPIC

et al.

e.V.

FEV1

$\mathrm{FiO}_{2}$

FRC

$\mathrm{HCO}_{3}$

I:E

IMC

ISB

IVC

k

$\mathrm{kPa}$

I

LGMD

MRSA

$\mathrm{mmHg}$

mmol

MS

ms
Signifikanzniveau

Amyotrophe Lateralsklerose

Akute respiratorische Insuffizienz

Blutgasanalyse

chronic obstructrive pulmonary disease

Kohlendioxid

Deutsche Interdisziplinäre Gesellschaft für Außerklinische

Beatmung

Escherichia coli

European Prevalence of Infection in Intensive Care

et alii

eingetragener Verein

Einsekundenkapazität (forced expiratory volume in one second)

inspiratorische $\mathrm{O}_{2}$-Konzentration

funktionelle Residualkapazität

Hydrogencarbonat (Bicarbonat)

Verhältnis Inspiration zu Exspiration

Intermediate Care Unit

intermittierende Selbstbeatmung

inspiratorische Vitalkapazität

Einzelhypothese

Kilopascal

Liter

Gliedergürtelmuskeldystrophie (limb-girdle muscular

dystrophy)

Methicillin-resistenter Staphylococcus aureus

Millimeter Quecksilbersäule

Millimol

multiple Sklerose

Millisekunde 
eine Patientenaufnahme bis zu ihrer Entlassung von der Intensivstation (ein Patientenfall)

NIV noninvasive ventilation (nicht-invasive Beatmung)

NME neuromuskuläre Erkrankung

$\mathrm{O}_{2}$ OHS Sauerstoff

o.J.

p

P0,1

P0,1 max

$\mathrm{pCO}_{2}$

PCV

$\mathrm{PI}_{\max }$

$\mathrm{pO}_{2}$

r

RICU

$\mathbf{R}$ tot

RV

SD

SE

SMA

ssp.

Staph. aureus

TLC

VAP

VCV
Obesitas-Hypoventilationssyndrom

ohne Jahresangabe

Irrtumswahrscheinlichkeit

Mundverschlussdruck 100 ms nach Inspirationsbeginn maximaler Mundverschlussdruck 100 ms nach Inspirationsbeginn

Kohlendioxidpartialdruck

pressure controlled ventilation (druckkontrollierte

Beatmung)

maximaler statischer Inspirationsdruck

Sauerstoffpartialdruck

Korrelationskoeffizient

Respiratory Intensive Care Unit

totaler Atemwegswiderstand (resistance)

Residualvolumen

Standardabweichung

Standardfehler

Spinale Muskelatrophie

subspecies (Unterart)

Staphylococcus aureus

totale Lungenkapazität

ventilator-associated-pneumonia (Beatmungspneumonie)

volume controlled ventilation (volumengesteuerte, vollständig mechanische Ventilation) 


\section{Einleitung}

\subsection{Die Intensivstation}

Die Intensivstation wurde geschaffen, um bei Patienten in einem schweren bis lebensbedrohlichen Zustand qualifiziert und professionell durch spezifische Maßnahmen das Überleben zu sichern und eine adäquate Therapie einzuleiten (Lawin 1998). Um die kritische Phase der schwerst Erkrankten zu überbrücken, werden unter ständiger Überwachung intensive lebenserhaltende Maßnahmen ergriffen. Dazu gehört das Aufrechterhalten eines suffizienten Herzminutenvolumens durch eine angemessene Flüssigkeits- und Perfusionsdrucktherapie sowie eine Elektrotherapie bei Herzrhythmusstörungen, einer Nierenersatztherapie bei gegebener Indikation und einer Lungenersatztherapie in der Regel durch Beatmung. Auf der Intensivstation spielen Übertragungen von Keimen von einem zum anderen Patienten -sprich nosokomiale Infekte- eine große Rolle (Kerwat et al. 2010). Maßgeblich tragen zu diesem Infektionsrisiko invasive Zugänge bei, die für die Überwachung und Behandlung gelegt werden, sowie die Sedation bei maschineller Beatmung (Gastmeier et al. 1999).

\subsection{Infektionen auf Intensivstationen}

Im gesamten klinischen Alltag hat die ansteigende Zahl von nosokomialen Infektionen eine immer größere Bedeutung. Geffers et al. (2002) definieren sie als Infektionen, die weder zum Zeitpunkt der Aufnahme in das Krankenhaus existierten noch sich in ihrer Inkubationsphase befanden. Mit inbegriffen sind auch solche Infektionen, die zur Zeit der Hospitalisierung zwar erworben wurden, sich aber erst nach Entlassung des Patienten manifestieren.

\subsubsection{Häufigkeit}

Im Rahmen einer nationalen Querschnittsstudie von Gastmeier und Geffers (2008) wurde eine Prävalenz der nosokomialen Infektionen von 3,5\% auf Normalstation bestimmt. Angewendet wurde die international verbreitete Definition nosokomialer Infektionen des Centers for Disease Control and Prevention von Glenister et al. 
(1993). Man kann insgesamt davon ausgehen, dass in Deutschland ca. 400000 bis 600000 Patienten pro Jahr betroffen sind. Im Vergleich hierzu ist die Prävalenzrate auf deutschen Intensivstationen von 15,3\% wesentlich höher (Geffers et al. 2002). Laut EPIC-Studie (Vincent et al. 1995), welche die Prävalenz in 17 westeuropäischen Ländern untersuchte, liegt diese mit $21 \%$ noch höher. Die häufigsten Infektionen waren: Pneumonien (46,9\%), Infektionen der unteren Atemwege $(17,8 \%)$, Harnwegsinfekte (17,6\%) sowie Bakteriämien und Fungämien (12\%).

Je nach Studie erleiden bis zu 30\% der Patienten einer Intensivstation mindestens eine nosokomiale Infektion, welches mit längeren Liegedauern, erhöhten finanziellen Kosten und der Steigerung der Letalität verbunden ist (Vincent 2003).

\subsubsection{Entstehung}

Nosokomiale Infektionen sind wie die meisten bakteriellen Infektionen Selbstinfektionen aus der bestehenden Körperflora. Diese pathologische Besiedlung geschieht auf zwei Wegen: Zum einen auf dem endogenen Wege, bei dem körpereigene Erreger durch Translokation in normalerweise sterile Bereiche zunächst zu einer Kolonisation und dann zur Infektion führen. Dies wäre z.B. bei einer Pneumonie durch aszendierende gastrointestinale Flora während der Beatmung der Fall. Zum anderen gibt es die exogene nosokomiale Übertragung, die durch körperfremde Erreger von anderen Patienten, über Hände des Personals oder Gegenstände in der Umgebung hervorgerufen wird. Endogene Krankenhausinfektionen sind schwieriger zu vermeiden als exogene. Mehr als die Hälfte aller nosokomialen Infektionen lassen sich auch mit den besten Methoden der Krankenhaushygiene nicht verhindern (Grundmann et al. 2005).

$\mathrm{Ob}$ es bei einer Erregertransduktion auch zu einer Infektion kommt, kann vom $\mathrm{Pa}$ tienten selbst, also seinem Immunstatus, seinem Allgemeinzustand und Ernährungszustand abhängen. Die meisten Infektionen sind jedoch katheterassoziiert (Geffers et al. 2002). Hierunter fallen Katheter wie Blasen-, Gefäß- und Periduralkatheter.

Die Situation auf der Intensivstation ist eine besondere. Zunächst ist eine erhöhte Exposition durch intensiven Kontakt zwischen Patient und Personal bei hohem Pflegeaufwand (Scheithauer et al. 2009) und die Durchführung von vielen invasiven, 
diagnostischen und therapeutischen Maßnahmen zu nennen, die eine Infektion begünstigen. Durch den Einsatz von Breitspektrumantibiotika wird die Selektion und Ausbreitung von resistenten Krankheitserregern verstärkt (Vincent 2003). Außerdem wird eine große Zahl der Patienten beatmet, was mit einer Anwendung von Medikamenten vergesellschaftet ist, die zur Ruhigstellung des Beatmeten führen. Eine Nebenwirkung dieser Medikamente ist die Beeinträchtigung des Immunsystems (Köhler 2002). Die Sedierung hat zur Folge, dass muköse Sekrete, die den Nährboden für Erreger darstellen, nicht mehr abgehustet werden können und im Atmungstrakt verbleiben (Köhler 2002). Auch Flüssigkeit, die sich oberhalb der Tubusmanschette ansammelt, stellt einen derartigen Nährboden dar. Zudem begünstigt das Kunststoffmaterial des Endotrachealtubus die Adhäsion von pathogenen Keimen und der Tubus an sich schützt nicht vor Aspiration, weil der Cuff deutlich unter den Stimmbändern liegt, sodass es zur Verschleppung von Keimen in den Respirationstrakt kommt.

\subsubsection{Nosokomiale Pneumonien}

Nosokomiale Pneumonien können beim spontan atmenden Patienten, bei nicht-invasiver Beatmung und unter invasiver Beatmung entstehen. Die sogenannte Beatmungspneumonie oder ventilator-associated-pneumonia, kurz VAP, ist eine spezielle Bezeichnung für eine nosokomiale Pneumonie, die sich unter invasiver Beatmung entwickelt hat.

Die absolute Inzidenz der nosokomialen Pneumonien in Deutschland kann auf 120.000 pro Jahr geschätzt werden (Ewig 2011), eine US-amerikanische Erhebung beschreibt ein Auftreten von 5 bis 15 bei 1000 Krankenhausaufnahmen. Die VAPInzidenzrate liegt für den Auswertungszeitraum 2004-2008 nach Angaben des nationalen Referenzzentrums für nosokomiale Infektionen (NRZ 2009) bei 5,31 Pneumonien pro 1000 invasiven Beatmungstagen und stellt mit 46,9\% die häufigste aller nosokomialer Infektionen in der Intensivmedizin dar (Vincent et al. 1995).

Dembinski und Rossaint (2008) fanden heraus, dass das Risiko für eine Beatmungspneumonie bei invasiver Beatmung wesentlich mit der Beatmungsdauer korreliert: In den ersten fünf invasiven Beatmungstagen erhöht sich das Risiko annähernd um bis zu $3 \%$ und mit jedem weiteren um $1 \%$ pro Tag. $90 \%$ aller ventilato- 
rassoziierten Pneumonien entstehen in den ersten 10 Beatmungstagen. Die Gesamtletalität bei Patienten mit VAP liegen zwischen 30 und 70\% (American Thoracic Society und Infectious Diseases Society of America 2005), und die Länge des intensivmedizinischen Aufenthaltes steigt durch eine Pneumonie laut Safdar et al. (2005) signifikant im Durchschnitt um 6 Tage. Dieser Sachverhalt spielt auch in finanzieller Hinsicht eine enorme Rolle, da die zusätzlichen Kosten durchschnittlich mit ca. 7.300 Euro bzw. 10.000 bis 40.000 US-Dollar pro VAP veranschlagt werden (Safdar et al. 2005, Dietrich et al. 2002).

\subsubsection{Präventionsmaßnahmen}

Laut Robert Koch-Institut (Kramer et al. 2000) stellt die wichtigste Standardhygienemaßnahme auf der Intensivstation - wie auch auf der Normalstation - die Händedesinfektion mit einem alkoholischen Händedesinfektionsmittel dar. Weitere Maßnahmen sind der Handschuhwechsel unmittelbar nach Beendigung der Tätigkeit am Patienten, die möglichst kurze Verweildauer von Fremdkörpern wie Venenkathetern, Blasenkathetern, arteriellen Kathetern und Hirndruckmesssonden sowie die regelmäßige Prüfung ihrer Indikation. Auch sollten hygienisch einwandfreie interventionelle und pflegerische Techniken zur Verhütung von Blasenkatheterinfektionen, Venenkatheterinfektionen, Pneumonien bei Beatmung und postoperativen Wundinfektionen angewendet werden. Bei der Antibiotikatherapie und -prophylaxe sollte eine sorgfältige Indikation gestellt werden. Eine perioperative Antibiotikaprophylaxe über die Dauer des Eingriffs hinaus ist oft überflüssig, teuer und resistenzentwicklungsfördernd.

Im Hinblick auf das ärztliche Personal sollten regelmäßige Schulungen stattfinden und speziell ausgebildetes Pflegepersonal eingesetzt werden.

Medizinprodukte müssen sicher aufbereitet sowie sinnvolle wie auch gezielte Reinigungs- und Desinfektionsmaßnahmen ergriffen werden (Dettenkofer und Meyer 2011).

Beatmungsgeräte und -zubehör müssen regelmäßig nach einem bestimmten Schema gesäubert, desinfiziert und gewechselt werden. Auch sollte eine regelmäBige orale, pharyngeale und wenn möglich auch subglottische Absaugung beim Patienten durchgeführt werden. 
In Bezug auf die Tracheostomapflege sollte auch hier auf eine regelmäßige Reinigung unter aseptischen Bedingungen und eine gute Pflege der angrenzenden Haut geachtet werden (Kappstein 2009).

Zusammenfassend ist zu sagen, dass die Hygienevorgaben auf der Intensivstation besonders hoch sind, um die hohe Prävalenz von Infektionen und den damit verbundenen wesentlich schlechteren Outcome der Patienten zu verbessern.

\subsection{Die „Beatmungsintensivstation“}

Hinsichtlich der Prävention von Infektionen bei beatmeten Patienten sollte laut der Kommission für Krankenhaushygiene und Infektionsprävention beim Robert KochInstitut (Unertl und Heininger 2000) eine nicht-invasive Beatmung bevorzugt oder, wenn dies nicht möglich ist, eine frühe Tracheotomie durchgeführt werden. Nava et al. (1998) haben gezeigt, dass die absolute Überlebensrate um etwa $20 \%$ steigt, wenn es gelingt, durch eine frühzeitige nicht-invasive Beatmung eine Intubation zu vermeiden. Die Wahrscheinlichkeit einer nosokomialen Pneumonie reduziert sich erheblich. Werden invasiv beatmete Patienten an eine Maskenbeatmung transferiert, so steigt die Überlebensrate ebenfalls um absolute 20\%. Nosokomiale Pneumonien traten in der Untersuchung von Nava et al. im nicht-invasiv beatmeten Patientenkollektiv keine auf, im invasiv beatmeten Kollektiv dagegen in 20\% der Fälle. Somit ist das Risiko einer nosokomialen Infektion bei Vermeidung invasiver Beatmung wesentlich geringer. Der Einfluss einer frühzeitigen Tracheotomie auf die Infektionsrate ist dagegen nicht gesichert (Wang et al 2011).

\subsubsection{Die nicht-invasive Beatmung}

Bei der nicht-invasiven Beatmung, kurz NIV (noninvasive ventilation) handelt es sich um eine Beatmung über eine dicht sitzende Mund-, Nasen- oder Mund-Nasenmaske. Wie über den Endotrachealtubus oder die Trachealkanüle können grundsätzlich alle Beatmungsmodi von CPAP bis zur voll kontrollierten Beatmung auch über Masken eingesetzt werden.

Laier-Groeneveld et al. (1990) verstehen die nicht-invasive Beatmung als vollständig passive Beatmung zur Entlastung der inspiratorischen Atmungsmuskulatur, die 
intermittierend und später im Wechsel mit Spontanatmung vom Patienten oder dessen Betreuern selbst angewendet werden kann. Hier ist keine oder eine geringe Sedierung erforderlich. Laier-Groeneveld et al. haben den Begriff nicht-invasive oder invasive intermittierende Selbstbeatmung, kurz ISB, gewählt. Bei der invasiven ISB wird der Patient über eine Trachealkanüle statt über eine Maske beatmet, die sonstige Handhabung ist jedoch identisch.

Für eine intermittierende Selbstbeatmung stehen verschiedene Techniken zur Verfügung. Zunächst war in den fünfziger Jahren des 20. Jahrhunderts während der Polioepidemien die Negativdruckbeatmung bei ventilatorisch insuffizienten Patienten von großer Bedeutung (Drinker und McKhann 1986). Durch den „Tankventilator“ oder die „eiserne Lunge“ wurde ein extrathorakaler Unterdruck aufgebaut, welcher zur thorakalen Expansion führte und somit die Inspiration zur Folge hatte. Durch Überdruck konnte auch die Ausatmung unterstützt werden. Eine Sedation war bei dieser Beatmungstechnik nicht notwendig.

Aufgrund des deutlich geringeren technischen Aufwands nutzt man heute Überdruckbeatmungsgeräte wie zur invasiven Beatmung über den Endotrachealtubus. Hierfür werden portable Beatmungsgeräte mit geringerem technischen Aufwand (Schönhofer und Sortor-Leger 2002) sowie einfacherer Handhabung (Bunburaphong et al. 1997) benutzt, um sich besser auf die Adaptation an den Patienten konzentrieren zu können.

Um auf die individuelle Situation des Patienten einzugehen, kann man zwischen einem voll kontrollierten, einem assistiert-kontrollierten oder assistierten Beatmungsmodus wählen. Diese belüften die Lunge über Druck- oder Volumenvorgabe im Sinne einer Positivdruckbeatmung.

\subsubsection{Unterschiede gegenüber invasiver Beatmung}

Bei einer akuten Atmungsinsuffizienz wird als Therapie meist die invasive Beatmung mittels Endotrachealtubus gewählt, da die Voraussetzung für eine ISB ein wacher, adäquat reagierender Patient ist, der auch die Beatmungsmaske toleriert. Außerdem müssen die logistischen Voraussetzungen zur Durchführung und Überwachung gegeben sein (Kuhlen und Dembinski 2008). Studien bei vielen Erkrankungen sprechen jedoch dafür, dass eine nicht-invasive Beatmung genauso gut geeignet ist, um einen bedrohlichen Gesundheitszustand zu überbrücken. 
Die NIV wird dann alternativ zur Intubation genutzt. Gelingt die nicht-invasive Beatmung, so kommt es zu einer deutlich besseren Überlebensrate von mindestens 20\% (Brochard et al. 1995).

Die NIV bringt den Vorteil mit sich, dass unmittelbaren Schädigungen des Kehlkopfes und der Trachealschleimhaut als Folge der Intubation vorgebeugt werden. Auch hämodynamische Interaktionen und schwere Schädigungen auf alveolärer Ebene, welche zu Baro-, Volu- und Atelekttraumata mit Auswirkungen auf den gesamten Organismus führen, können laut Plötz et al. (2004) vermieden werden.

Speziell in der Langzeittherapie machen sich die Vorteile einer NIV bemerkbar. Schönhofer et al. (2008) zeigen auf, dass die tubusbedingte zusätzliche Atmungsarbeit wegfällt und die Entwöhnung vom Respirator wesentlich erfolgreicher ist. AuBerdem kann auf Sedativa verzichtet werden, die eine gute Kommunikation wie auch Essen und Trinken verhindern würden.

Dem gegenüberzustellen wäre laut Carlucci et al. (2001) eine hohe Abbruchrate der NIV aufgrund infrastruktureller Mängel, unzureichendem technischen Equipment und der geringen Erfahrung des Personals. Zudem beschreiben Schönhofer et al. (2008) Druckstellen der Maske im Gesichtsbereich, eine vermehrte $\mathrm{CO}_{2}$-Rückatmung, mehr oder weniger ausgeprägte Leckagen und häufigere Aerophagien.

Besonders deutlich ist die Überlegenheit der NIV im Bezug auf eine geringere Infektionsgefahr.

Dieser herausragende Vorteil kommt dadurch zustande, dass sich zum einen kein Fremdkörper in Form eines Endotrachealtubus im Respirationstrakt des Patienten befindet. Es kann sich keine Flüssigkeit oberhalb der Tubusmanschette ansammeln, die ein idealer Nährboden für pathogene Erreger wäre, und die Adhäsion von Keimen an das Kunststoffmaterial des Tubus und die darauf folgende Absiedlung in tiefere Bronchialabschnitte entfällt. Eine Intubation mittels Endotrachealtubus stellt keinen ausreichenden Schutz vor Mikroaspiration dar. Dies führt zu einer Verschleppung von Keimen in die Lunge und zu tubusassoziierten Pneumonien.

Zum anderen ist bei einer NIV keine Sedation des Patienten zur Duldung des Endotrachealtubus notwendig. Dadurch wird dem Patienten ein eigenständiges Abhusten von Schleim ermöglicht, und physiologische Barrieren, wie die mukozilliäre Clearance, werden in ihrer Funktion nicht gestört (Schönhofer et al. 2008; Köhler 2002; Thomas 1997). 
Zusammenfassend werden die invasiven und nicht-invasiven Beatmungsverfahren in der Therapie einer akuten respiratorischen Insuffizienz je nach Erfahrung alternativ eingesetzt (Burchardi und Schönhofer 2000). Die NIV führt aber zur Senkung der Komplikationsrate, zur schnelleren Mobilisation des Patienten und zur Verkürzung des Krankenhausaufenthaltes. Sie vermindert besonders die nosokomialen Infektionen und die damit verbundenen tiefgreifenden Folgen (Peter et al. 2002).

\subsection{Die praktische Umsetzung einer „Beatmungsintensivstation“ im Klinikum Niederrhein}

Die Respiratory Intensive Care Unit (RICU) im Klinikum Niederrhein ist eine der Intensivstationen, in der fast ausschließlich die ISB als Beatmungstherapie bei schwerst Lungenerkrankten sowohl nicht-invasiv über Maske als auch invasiv über Tubus oder Tracheostoma angewendet wird. Daher ist eine deutlich geringere Rate an nosokomialen Erkrankungen zu erwarten.

Bei dieser Beatmungsform kommen besondere Hygienekriterien zum Tragen, welche die Deutsche Interdisziplinäre Gesellschaft für Außerklinische Beatmung (DIGAB), ehemals Arbeitsgemeinschaft Heimbeatmung und Respiratorentwöhnung e.V. (Laier-Groeneveld 1996), formuliert hat und die demgemäß im Klinikum Niederrhein umgesetzt werden. Kerninhalte sind, dass sauber, aber nicht steril gearbeitet werden muss. Die Händedesinfektion steht im Vordergrund, aber sterile Handschuhe sind nicht notwendig. Absaugen ohne Handschuhe ist aus rein infektiologischen Gründen möglich, wird zum Selbstschutz vor Sekret jedoch nicht empfohlen. Beatmungszubehör soll sauber gehalten werden, aber nicht steril, sowohl im stabilen Zustand als auch während eines Infektes. Bakterienfilter sind nirgendwo notwendig. Beatmungszugänge, Trachealkanülen, Masken und Beatmungssysteme können wiederverwendet werden und sollen mit Wasser gesäubert, aber nicht desinfiziert werden.

Während des Untersuchungszeitraumes, sprich im Jahre 2010, bestand die RICU des Johanniter Krankenhauses Oberhausen aus einem 4-Bettzimmer. In diesem 
überwachte an einer zentralen Pflegestation in Form eines Schreibtisches eine Pflegekraft die Patienten. Hier wurden auch gleichzeitig administrative Arbeiten verrichtet. Erst in den Folgejahren wurde die Station auf 13 Betten aufgerüstet.

\subsubsection{Anwendungsgebiete der ISB}

Auf der RICU des Klinikums Niederrhein wird die ISB bei drei verschiedenen Behandlungsindikationen angewendet. Diese sind das "Weaning", die „Behandlung bei akuter Atmungsinsuffizienz“ und die Einleitung sowie Kontrolle einer „Intermittierenden Selbstbeatmungstherapie“.

Der englische Begriff Weaning, auf Deutsch Entwöhnung, beschreibt die Phase der Wiederherstellung der Spontanatmung einer meist per Trachealtubus beatmeten Person und ist ein dynamischer Prozess. Eine invasive maschinelle Atmungsunterstützung findet meistens im Rahmen einer intensivmedizinischen Behandlung oder Narkose während einer Operation statt. Bei kurzen Beatmungsdauern von Stunden bis wenigen Tagen ist die Beatmungsentwöhnung oft unproblematisch, wohingegen längere Beatmungszeiträume ein komplizierteres „Weaning“ mit sich bringen, welches z.B. Nava et al. (1994) nachwiesen: Nach drei Wochen invasiver Beatmung von 42 Patienten, die an COPD erkrankt waren, konnten 19 Personen, fast die Hälfte (45\%), nicht entwöhnt werden. Auch wirkte sich die lange Beatmungsdauer bei intubierten Patienten negativ auf die Mortalität aus. Die Überlebensrate der länger beatmeten Patienten war mit 22\% signifikant niedriger, als die der 23 anderen mit 68\% (Nava et al. 1994).

Um die Atmungsarbeit Schritt für Schritt vom Beatmungsgerät auf den Patienten zurück zu übertragen, ist es nötig, die Sedierung des Patienten zu reduzieren und bei komplizierteren Verläufen kann eine perkutane Tracheotomie als Erleichterung dienen (Jantzen 2008). Auch wird empfohlen, sich für diesen Prozess die assistierte Spontanatmung in Form von BIPAP („Biphasic Positive Airway Pressure“) und danach CPAP („Continuous Positive Airway Pressure“) zu Hilfe zu ziehen (Kuhlen und Dembinski 2008). Auf der RICU des Klinikums Niederrhein wird eine atemmuskulaturentlastende statt einer lungenprotektiven Strategie verfolgt: Es wird die passive ISB durchgeführt. Dies impliziert, dass der Patient für eine

bestimmte Phase 
passiv beatmet wird, also nicht selbst mitatmet, gefolgt von einer Phase vollständiger Eigenatmung. Laier-Groeneveld et al. (1989b) konnten im Hinblick darauf schon 1989 durch fünf Kasuistiken zeigen, dass nach einer Langzeitbeatmung zwischen 57 und 112 Tagen über Tracheostoma ein Übergang auf eine nicht-invasive Beatmung möglich war.

Eine weitere Funktion der RICU ist die Behandlung der akuten Atmungsinsuffizienz durch eine ISB, welche für diese Indikation national z.B. von Burchardi et al. (2002), aber auch international durch die International Consensus Conferences in Intensive Care Medicine (2001) empfohlen wurde.

Schon 1989 beschreiben Meduri et al. eine erfolgreiche nicht-invasive ventilatorische Behandlung von zehn Patienten mit hypoxischer wie auch hyperkapnischer ARI.

Hierbei stellt die häufigste Ursache die exazerbierte COPD dar, in deren Rahmen es zur drohenden Erschöpfung der Atmungsmuskulatur kommt. Die ISB verhindert dies durch die Entlastung des Atmungsapparats und steigert die Ventilation (Schönhofer 2008), sodass im Vergleich zur konventionellen Therapie laut Lightowler et al. (2003) das relative Risiko der Letalität auf 0,41 und das relative Risiko der Intubationsfrequenz auf 0,42 verringert wird.

Das Kardiale Lungenödem beschreiben Winck et al. (2006) als eine weitere klare Indikation der ISB.

Als drittes sei die Einleitung und Kontrolle einer dauerhaften ISB zu benennen. Im Zusammenhang damit ist der Begriff „Intermittierende Selbstbeatmungstherapie“, kurz „ISB-Therapie“, von Laier-Groeneveld et al. (1989a) geprägt worden: Invasiv beatmete Patienten, deren Entwöhnung im weiteren Verlauf vom Respirator erfolglos bleibt, sowie diejenigen Lungenerkrankten, bei denen aufgrund von Funktionsstörungen des Respirationstraktes der Sauerstoffbedarf für den Organismus von Grund her nicht eigenständig gedeckt werden kann und Kohlenstoffdioxid nicht ausreichend abgeatmet wird, erhalten eine für eine bestimmte Zeitdauer pro Tag begrenzte nicht-invasive Beatmung. Die ISB stellt somit eine Therapie der zugrundeliegenden Erkrankung nach Laier-Groeneveld et al. (1989b) dar und kann auch im häuslichen Bereich fortgeführt werden. 
Patienten mit fortgeschrittener COPD, neuromuskulären Erkrankungen, wie Amyotrophe Lateralsklerose und Muskeldystrophie Duchenne, Thoraxwanddeformitäten u. v. a. profitieren von dieser Therapie, indem die Atmungsmuskulatur intermittierend entlastet wird und die $\mathrm{CO}_{2}$-Schwelle des Atemzentrums von hyperkapnischen Werten wieder die Norm erreicht (Laier-Groeneveld et al. 1997).

Weitere Studien zeigten eine Verbesserung der Lungenfunktion sowie Vitalkapazität (Laier-Groeneveld et al. 1989a). Simonds und Elliott (1995) beschrieben, dass selbst bei alleiniger Anwendung der ISB bei Nacht sich die Blutgase unter Spontanatmung am Tag normalisierten.

Positiv hervorzuheben ist auch, dass sich der Patient nach Laier-Groeneveld (1998) nicht als beatmungsabhängig sieht, sondern als beatmungsunterstützt. In einer randomisierten kontrollierten Studie zeigen Köhnlein et al. (2014), dass nicht nur die Langzeitlebenserwartung, sondern auch die Lebensqualität gesteigert wird. Dies belegen auch Léger et al. (1994), welche subjektive klinische Zeichen untersuchten und eine verbesserte Konzentrationsfähigkeit und reduzierte Tagesmüdigkeit beobachteten.

\subsubsection{Praktische Umsetzung der Beatmungstherapie}

Im Gegensatz zu einer üblichen Intensivstation wurde in Bezug auf die Räumlichkeiten statt einer strengen Isolation ein offenes Setting in Form eines Vierbettzimmers auf der RICU Oberhausen gewählt. Nava et al. (1998) argumentieren, dass zum einen dadurch die Angehörigen das meist umfangreiche Pflegeprogramm unterstützen können und zum anderen eine Pflegekraft mehrere Patienten gleichzeitig versorgen kann. Zusätzlich wurde die effektive Nutzung des Pflegepersonals, dessen Zeitaufwand v.a. bezüglich der Dokumentation immer weiter zunimmt, dadurch gefördert, dass sich im Patientenzimmer eine zentrale Pflegestation in Form eines Schreibtisches für administrative Arbeiten befand, um das gleichzeitige Überwachen in direkter Nähe zu den Patienten zu gewährleisten (Laufman 1986). Außerdem war es möglich, individuell je nach Bedarf und auf eigenen Wunsch der Patienten, sie von pharyngealem, laryngealem und trachealem Sekret mittels bronchoskopischer Absaugung zu befreien, welches, wie schon oben beschrieben, eine wichtige Maßnahme zur Eindämmung von Infektionen und Keimvermehrungen darstellt. 
Der klassische Pflegeschlüssel auf einer Intensivstation liegt bei 1:1 (Elpern et al. 1991) bzw. sollte eine Pflegekraft nach Corrado et al. (2002) pro Schicht nicht mehr als zwei Patienten versorgen. Die Literatur spricht von drei bis fünf Patienten, die von einer Fachkraft auf der RICU gleichzeitig gepflegt werden (Elpern et al. 1991). 24 Stunden pro Tag solle diese Pflegekraft anwesend sein (Corrado et al. 2002). Auch im Johanniter Krankenhaus war durchgängig Pflegepersonal anwesend und die vier Patienten wurden von einer speziell ausgebildeten Pflegefachkraft in einer Relation von 1:4 versorgt. Vom Robert-Bosch-Krankenhaus Stuttgart (Gorgs 2013) oder dem Uniklinikum Ulm (Ickler 2011) werden seit 2007 hierfür eine mindestens 200 Stunden umfassende Pflegefachweiterbildung für die Intensivstation angeboten. Die Krankenpflegeschule Duisburg e.V. führt u.a. für Pflegekräfte auf der RICU Fachweiterbildungen zu „Pflegeexperten für außer-klinische Beatmung“ durch, die insgesamt 280 Stunden in Theorie und Praxis beinhalten (Topel o. J.). 


\subsection{Fragestellung}

Es fällt auf, dass sich die RICU hinsichtlich des Patientenmanagements und der Präventionsmaßnahmen gegen nosokomiale Infektionen von einer üblichen Intensivstation unterscheidet: Die Patienten werden bei Aufnahme direkt extubiert und intermittierend selbst beatmet, invasive Zugänge werden schnell entfernt und die Patienten erhalten weniger Medikamente. Vor allem Sedativa fallen mit der Aufnahme vollständig weg. Die Absaugung, die nach Wunschäußerung des Patienten und daher wesentlich häufiger stattfindet als auf einer üblichen Intensivstation, erfolgt unter sauberen, aber nicht sterilen Bedingungen.

In Anbetracht dieses besonderen Settings auf der RICU kann von einer erheblichen Risikoreduktion für eine nosokomiale Infektion ausgegangen werden. Es liegen jedoch weltweit keine vergleichbaren Daten vor, die beschreiben würden, inwieweit Infektionsrisiken, die auf einer üblichen Intensivstation vorliegen, auch eine Rolle auf der RICU spielen. Daher soll diese Arbeit anhand der Patienten, die im Jahre 2010 auf der RICU im Johanniter Krankenhaus Oberhausen behandelt wurden, untersuchen, in welchem Rahmen es bei den veränderten Grundvoraussetzungen auf der RICU zu Keimübertragungen von einem Patienten zum anderen kommt und welche Bedeutung die Übertragung auf Erkrankung, Therapie und Prognose in Abhängigkeit von der Grunderkrankung und des Schweregrades, definiert durch Lungenfunktion, Atmungsfunktion und Blutgase, sowohl während des stationären Aufenthaltes als auch nach Entlassung aus der Klinik hat. Es ist folgend zu diskutieren, welche Konsequenzen die Ergebnisse in Bezug auf die angewendeten Hygienestandards haben: Sind die Infektionsrate und die damit zusammenhängenden Folgen für den Patienten auf der RICU akzeptabel oder sollten neue Hygienerichtlinien propagiert werden. 


\section{Material und Methoden}

\subsection{Patientencharakteristik}

\subsubsection{Einschlusskriterien}

Es wurden alle Patienten untersucht, die im Jahr 2010 auf die RICU des Johanniter Krankenhauses Oberhausen aufgenommen wurden. Eine Patientenaufnahme bis zu ihrer Entlassung von der Intensivstation wurde als ein Fall definiert. Wenn derselbe Patient zu einem späteren Zeitpunkt erneut stationär auf die RICU aufgenommen wurde, wurde dies als neuer Fall festgehalten. Die Patienten erklärten sich damit einverstanden, dass im Rahmen dieser Studie sie betreffende personenbezogene Daten / Angaben durch den Studienarzt erhoben, verschlüsselt (pseudonymisiert) auf elektronischen Datenträgern aufgezeichnet und verarbeitet werden dürfen. Auch willigten sie ein, dass die Studienergebnisse in anonymer Form veröffentlicht werden dürfen.

Die Studie wurde von der Ethikkommission der Medizinischen Fakultät der Universität Göttingen und der Ethikkommission der Ärztekammer Nordrhein geprüft. Den Anträgen wurde am 06.05.2013 bzw. 26.02.2015 stattgegeben.

\subsubsection{Grunderkrankungen und Ursache der Beatmung}

Die Grunderkrankungen, an denen die Patienten litten, wurden anhand objektiver Parameter, Lungen- und Atemfunktion, Blutgase etc. verifiziert. Patienten mit einer chronisch obstruktiven Lungenerkrankung (COPD) und Asthma bronchiale wurden den Fällen mit einer obstruktiven Grunderkrankung zugeordnet. Neuromuskuläre Erkrankungen, wie Amyotrophe Lateralsklerose (ALS), Muskeldystrophie Duchenne, Gliedergürtelmuskeldystrophie (LGMD), Spinale Muskelatrophie (SMA), multiple Sklerose (MS) und die Stoffwechselerkrankung Mukopolysaccharidose IV wurden zu den restriktiven Grunderkrankungen gezählt. Der Schweregrad wurde über die Lungen- und Atmungsfunktion gemessen.

Der Grund für den Beatmungsbeginn wurde aus der Gesamtschau des Behandlungsverlaufes festgelegt. 


\subsubsection{Liegedauer, Anzahl und Dauer der Patientenkontakte}

Als Liegedauer wurde die Zeitspanne zwischen Aufnahme- und Entlassungsdatum von der Intensivstation bestimmt und in ganzen Tagen erfasst. Ein Aufenthalt auf der Normalstation wurde nicht miteinbezogen. Der Tag der Aufnahme und Entlassung wurde jeweils als ein Tag angerechnet.

Jeder stationäre Aufenthalt eines Patienten auf der RICU, der sich mit dem eines anderen auf demselben Zimmer befindlichen Patienten für mindestens einen Tag überschnitt, wurde als ein Patientenkontakt definiert.

Die Summe der Tage mit zeitlich überschneidendem Aufenthalt zweier Patienten auf der RICU wurde als Kontaktdauer bestimmt.

Die Vorgehensweise der Zählung der Patientenkontakte und die Berechnung der Kontaktdauer sind in Grafik 2.1.3.1 dargestellt.

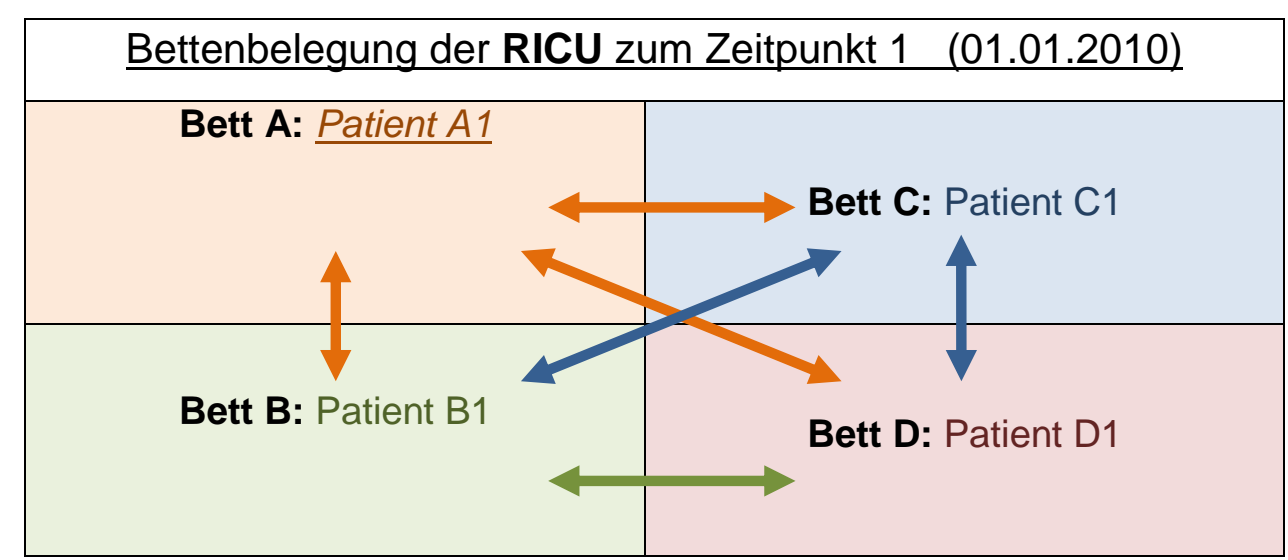

Grafik 2.1.3.1: beispielhafte Bettenbelegung der RICU zum Zeitpunkt 1

Legende: $\longleftrightarrow$ : Patientenkontakte zwischen Patienten A1 und B1, Patienten A1 und $\mathrm{C} 1$ und Patienten $\mathrm{A} 1$ und $\mathrm{D} 1$

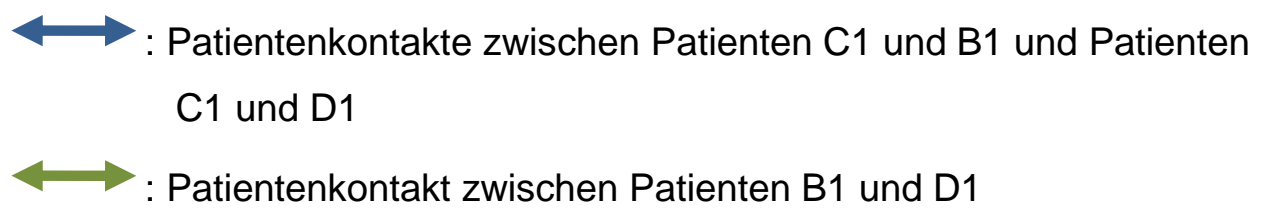

Die RICU ist eine Intensivstation bestehend aus einem Zimmer mit vier Betten. Zum Zeitpunkt 1 (siehe Grafik 2.1.3.1) befinden sich dort vier Patienten: „Patient A1“, „Patient B1", „Patient C1" und „Patient D1". Die Pfeile stellen die Patientenkontakte innerhalb des Patientenzimmers dar. Durch die orange gefärbten Pfeile werden die drei Kontakte beschrieben, die zwischen Patient $A 1$ und seinen

Bettnachbarn 
bestehen. Hinzuzufügen sind zwei Patientenkontakte, die durch blaue Pfeile gekennzeichnet sind, welche Patient C1 zu Patient B1 und Patient D1 aufweist. Schließlich besteht noch ein weiterer Patientenkontakt zwischen Patient B1 und Patient D1, der durch den grünen Pfeil symbolisiert wird. Insgesamt können sechs Pfeile: drei orangefarbene, zwei blaue und ein grüner, in der Grafik 2.1.3.1 aufsummiert werden. Diese symbolisieren sechs Patientenkontakte zum Zeitpunkt 1.

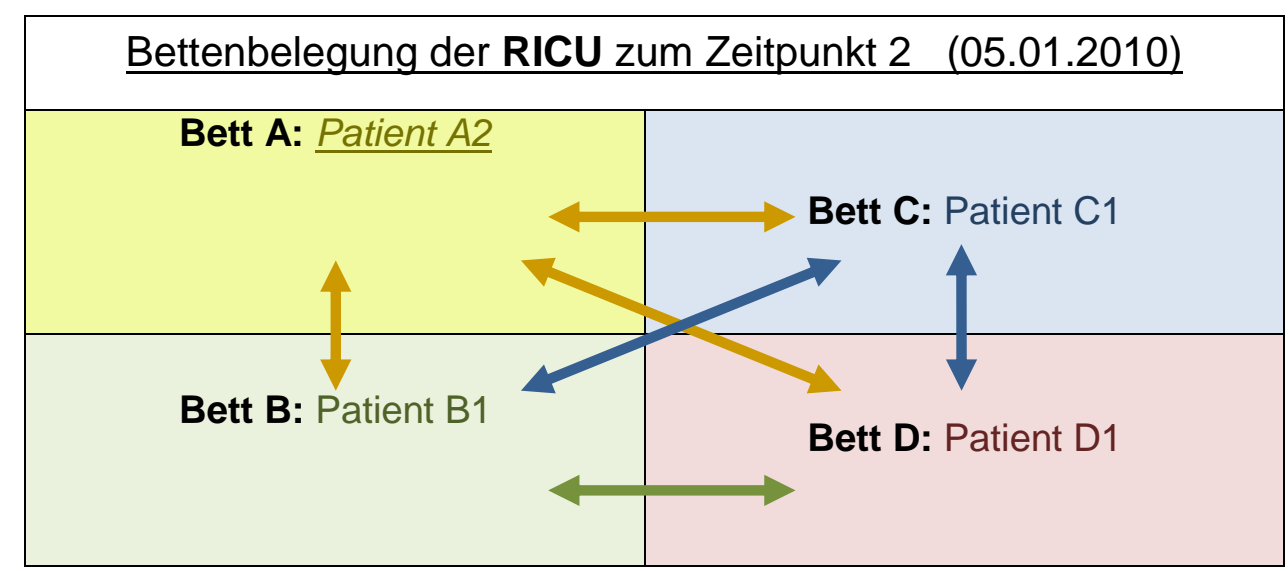

Grafik 2.1.3.2: beispielhafte Bettenbelegung der RICU zum Zeitpunkt 2

Legende: $\longrightarrow$ : Patientenkontakte zwischen Patienten A2 und B1, Patienten A2 und $\mathrm{C} 1$ und Patienten A2 und D1

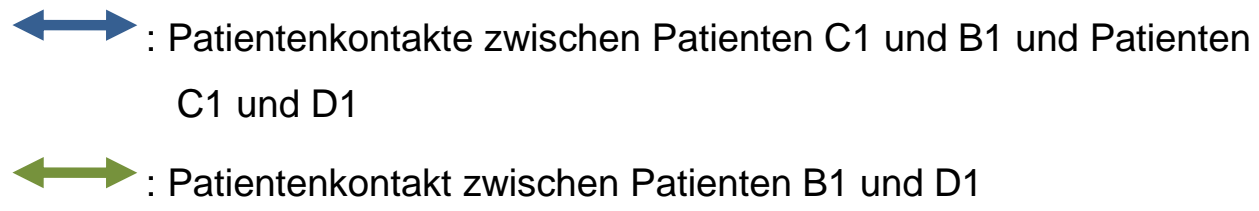

Zum Zeitpunkt 2 wurde Patient $A 1$ aus der RICU entlassen und ein neuer Patient Patient A2 aufgenommen, welcher sich in Bett A befindet (siehe Grafik 2.1.3.2). Die Patientenaufnahme hat zur Folge, dass drei weitere Patientenkontakte, die mit dunkel-gelben Pfeilen dargestellt sind, hinzu gekommen sind. Insgesamt kann man nun neun Patientenkontakte im Zeitraum vom 01.01.2010 (Zeitpunkt 1) bis zum 05.01.2010 zählen.

Von jedem Kontakt wurde auch dessen Dauer in Tagen erfasst. Der Patientenkontakt zwischen Patient $A 2$ und Patient $C 1$ begann zum Zeitpunkt 2, sprich am 05.01.2010. Geht man beispielsweise davon aus, dass Patient A2 am 10.01.2010 entlassen wurde, bestand der Kontakt vom 05.01.2010 bis 10.01.2010. Die Kontaktdauer beträgt demnach sechs Tage. 


\subsubsection{Mortalität}

Als Mortalität wurde die 90-Tage-Mortalität verwendet, wobei die 90 Tage ab dem Tag der Entlassung gezählt wurden. Das genaue Todesdatum verstorbener Patienten wurde anhand der hausinternen Kartei der Patienten bis auf den Tag genau ermittelt. War dies nicht bekannt, wurde ein direkter Kontakt mittels Telefonanruf zu Familienangehörigen aufgenommen.

\subsubsection{Keime und Infektionen}

Als Keimübertragung wurde der Nachweis des gleichen Bakterienstammes mit gleichem Antibiogramm bei zwei Patienten definiert, die sich zeitgleich auf der RICU befanden hatten. Der Keimnachweis fand mittels mikrobiologischer Untersuchungen von Sputum, Bronchialsekret oder arteriellem Blut statt. Es wurden je 25 Antibiotikaresistenzen getestet. Eine weitere Typisierung der Erreger erfolgte nicht.

Bei jedem Patienten wurden routinemäßig mindestens drei mikrobiologische Untersuchungen von Rachen- und Bronchialsekreten durchgeführt: bei Aufnahme, bei Entlassung, wöchentlich und im Rahmen von Bronchoskopien. Bei auffälliger Klinik folgten dementsprechend weitere mikrobiologische Untersuchungen. Ausgewertet wurden alle positiven mikrobiologischen Befunde.

Infektionen wurden vom behandelnden Arzt erfasst, wenn die Mindestvoraussetzungen für die jeweilige Infektion per Definition erfüllt waren. 


\subsection{Durchgeführte Untersuchungen}

\subsubsection{Blutgasanalysen}

Blutgasanalysen wurden bald möglichst nach Aufnahme und bei Entlassung jeweils unter Spontanatmung durchgeführt. Um akute Beatmungseffekte zu vermeiden, wurden die Werte zwei bis vier Stunden nach der letzten Beatmungsphase bei reiner Spontanatmung am Ende der Spontanatmungsphase bestimmt.

Arterielles Blut, das mit einer heparinisierten Spritze aus der Arteria radialis entnommen wurde, oder arterialisiertes Blut aus dem Ohrläppchen diente als Blutprobe. Die Untersuchung der Blutgase wurde direkt nach der Blutentnahme durchgeführt (CIBA CORNING 238 Blutgasanalysator, Medfield, MA, USA).

Referenzbereiche:

\begin{tabular}{|l|l|l|}
\hline Untersuchungswert & Referenzbereich & Einheit \\
\hline $\mathrm{pH}$ & $7,37-7,43$ & \\
\hline $\mathrm{pCO}_{2}$ & $35,00-45,00$ & $\mathrm{mmHg}$ \\
\hline $\mathrm{pO}_{2}$ & $71,00-104,00$ & $\mathrm{mmHg}$ \\
\hline $\mathrm{HCO}_{3}$ & $21,00-26,00$ & $\mathrm{mmol} / \mathrm{l}$ \\
\hline
\end{tabular}

\subsubsection{Funktionsuntersuchungen}

Nachfolgende Funktionsuntersuchungen wurden zur Beurteilung der Art und des Schweregrades der Grunderkrankung durchgeführt: Spirometrie, Bodyplethysmographie und Mundverschlussdruckmessung. Die Funktionsuntersuchungen fanden in stabilem Zustand unmittelbar vor Entlassung statt.

\subsubsection{Spirometrie und Bodyplethysmographie}

Pneumotachographisch wurden die Inspiratorische Vitalkapazität (IVC) und die Einsekundenkapazität (FEV1) gemessen und zusätzlich jeweils in Prozent des Sollwertes (IVC\% und FEV1\%) angegeben. Außerdem wurde das Verhältnis von Einsekundenkapazität zur inspiratorischen Vitalkapazität in Prozent angegeben (FEV1/IVC \%). Der totale Atemwegswiderstand oder Resistance (R tot), die totale 
Lungenkapazität (TLC) und das Residualvolumen (RV) wurden bodyplethysmographisch ermittelt und das Verhältnis von Residualvolumen zur totalen Lungenkapazität in Prozent angegeben (RV/TLC \%).

Alle Messungen erfolgten durch den Bodyplethysmographen JAEGER® MasterScreen® Body (Firma CareFusion, San Diego, California, USA).

\subsubsection{Mundverschlussdruckmessung}

Der Mundverschlussdruck 100 ms nach Inspirationsbeginn (P0,1), der maximale Mundverschlussdruck $100 \mathrm{~ms}$ nach Inspirationsbeginn (P0,1 max) sowie der maximale statische Inspirationsdruck ( $\mathrm{PI}_{\max }$ ) wurden als Parameter der Atmungsfunktion bestimmt. Als Messgerät wurde der ZAN Bodyscope verwendet (Firma ZAN Messgeräte $\mathrm{GmbH}$, Deutschland). Der Patient saß bei der Messung aufrecht, während die Nase durch eine Nasenklammer verschlossen war.

Die Größe des aktuellen Kraftaufwandes bei Ruheatmung bzw. das Maß der Last der Atmungspumpe wurde als P0,1 gemessen. Hierbei atmete der Patient bei spontaner Atmung in der Atemruhelage (FRC). In unregelmäßigen Zeitabständen wurde das Inspirationsventil zu Beginn der Inspiration für 180 ms verschlossen. Der Druck wurde $100 \mathrm{~ms}$ nach Inspirationsbeginn festgehalten und zur Vereinfachung, obwohl negativ, mit einem positiven Vorzeichen versetzt. Der P0,1 wurde als Durchschnittswert aus mindestens fünf Messungen bestimmt, um Schwankungen der Atmung auszugleichen (Laier-Groeneveld und Criée 1994).

Der PI $I_{\max }$ wurde als Maximalwert während eines maximal forcierten Inspirationsmanövers nach vorheriger Exspiration auf Residualvolumenniveau gegen ein komplett für 2 Sekunden verschlossenes Ventil ermittelt. Die Bestimmung vom P0,1 max erfolgte durch das gleiche Atmungsmanöver 0,1 Sekunden nach Inspirationsbeginn. Die Messung wurde mindestens fünfmal wiederholt, um mitarbeitsbedingte Effekte auszuschließen (Laier-Groeneveld und Criée 1994).

Der $\mathrm{PI}_{\max }$ wurde in $\mathrm{kPa}$ angegeben. Er stellt die maximale Kapazität und damit einen Index der maximalen Kraft der Atmungspumpe dar. Die Größe der Beanspruchung der Atmungspumpe bei Ruheatmung kann als Verhältnis von P0,1 zu P0,1 max gesehen werden. Es wurde als Prozentwert angegeben (P0,1/P0,1 $\max (\%))$. Auch das Verhältnis von $\mathrm{P} 0,1$ zu Pl max wurde in Prozent angegeben (P0,1/PI $\max (\%))$. Beide 
Quotienten kennzeichnen den aktuellen Kraftaufwand (Last) in Bezug zur maximalen Kraft (Kapazität).

Als Normwerte dienten die Empfehlungen der Deutschen Atemwegsliga zur Messung der inspiratorischen Muskelfunktion (Criée 2003).

\subsection{Statistische Auswertung}

Quantitative Merkmale wurden mit Mittelwert \pm Standardabweichung festgehalten. Der t-Test für unverbundene Stichproben wurde zur Berechnung von kontinuierlichen Daten von zwei zu untersuchenden Gruppen verwendet. Verlaufsbeobachtungen wurden mit dem t-Test für verbundene bzw. gepaarte Stichproben analysiert. Bivariate Korrelationen wurden mit Fisher's exact test errechnet und mit Irrtumswahrscheinlichkeit ( $p$ ), Korrelationskoeffizient $(r)$ und Signifikanzniveau $(\alpha)$ angegeben. Das Signifikanzniveau wurde konventionsgemäß mit $p<0,05$ definiert. Bei bivariaten Korrelationen bei multiplen Tests, die mit Fisher's exact test errechnet wurden, wurde die multiple a-Niveauanpassung nach Bonferroni hinzugezogen. Hierbei wird das Signifikanzniveau mit $p \leq \alpha / k$ definiert ( $k=$ Einzelhypothese).

Die erhobenen Daten wurden mit SPSS Statistics Version 21 verwaltet und analysiert. 


\section{Ergebnisse}

Es wurden im Jahr 2010 insgesamt 126 Patienten auf der RICU aufgenommen. Darunter waren 83 Männer und 43 Frauen. Das durchschnittliche Alter betrug 62 Jahre mit einer Standardabweichung von 17,37 Jahren.

Die durchschnittliche Dauer des stationären Aufenthalts auf der RICU betrug 11,44 Tage mit einer Standardabweichung von 13,51 Tagen.

\subsection{Grunderkrankungen und Ursachen der Beatmung}

Bei 91 Patienten lag eine obstruktive und bei 22 Patienten eine restriktive Grunderkrankung als führende Erkrankung vor. 12 Patienten wiesen eine andere Grunderkrankung auf.

Bei einer Patientin konnte die Grunderkrankung nicht klar definiert werden. Es handelt sich um eine 75-jährige Dame, die aufgrund einer Pneumonie respiratorisch insuffizient wurde. Sie verstarb am 22.04.2010 während ihres stationären Aufenthalts aufgrund der Erschöpfung der Atmungspumpe als Folge des Infekts. Eine Funktionsuntersuchung war daher nicht möglich.

Eine genaue Auflistung stellt Tabelle 3.1.1 auf der folgenden Seite dar. 


\begin{tabular}{|l|c|}
\hline & $\begin{array}{c}\text { alle Fälle } \\
\text { (n=126) }\end{array}$ \\
\hline keine definierte Grunderkrankung & $1(0,8 \%)$ \\
\hline obstruktive Grunderkrankungen & $91(72,2 \%)$ \\
\hline davon COPD & $90(71,4 \%)$ \\
\hline davon Asthma bronchiale & $1(0,8 \%)$ \\
\hline \hline restriktive Grunderkrankungen & $22(17,5 \%)$ \\
\hline davon Muskeldystrophie Duchenne & $12(9,5 \%)$ \\
\hline davon ALS & $4(3,2 \%)$ \\
\hline davon Gliedergürtelmuskeldystrophie & $1(0,8 \%)$ \\
\hline davon Spinale Muskelatrophie & $1(0,8 \%)$ \\
\hline davon Multiple Sklerose & $1(0,8 \%)$ \\
\hline davon Morbus Parkinson & $1(0,8 \%)$ \\
\hline davon Mukopolysaccharidose Typ IV & $1(0,8 \%)$ \\
\hline davon Tetraparese nach Basilarisaneurysma & $1(0,8 \%)$ \\
\hline \hline andere Grunderkrankungen & $12(9,5 \%)$ \\
\hline davon Lungenfibrose & $3(2,4 \%)$ \\
\hline davon Bronchialkarzinom & $2(1,6 \%)$ \\
\hline davon Herzinsuffizienz & $2(1,6 \%)$ \\
\hline davon zentrale Atemregulationsstörung & $2(1,6 \%)$ \\
\hline davon Z.n. Lungenteilresektion bei Bronchialkarzinom & $1(0,8 \%)$ \\
\hline davon OHS: Obesitas-Hypoventilationssyndrom & $1(0,8 \%)$ \\
\hline davon Z.n. Mammakarzinom & $1(0,8 \%)$ \\
\hline
\end{tabular}

Tabelle 3.1.1 Grunderkrankungen der 126 aufgenommenen Patienten mit absoluter Häufigkeit und prozentualer Verteilung 
Die durchschnittliche Dauer des stationären Aufenthalts der Patienten unterteilt nach deren Grunderkrankungen stellt Tabelle 3.1.2 dar.

\begin{tabular}{|l||c|}
\hline & $\begin{array}{c}\text { stationäre Aufenthaltsdauer } \\
\text { Mittelwert } \pm \text { Standardabweichung }\end{array}$ \\
\hline \hline keine definierte Grunderkrankung & $\begin{array}{c}54 \pm 0 \text { Tage } \\
(n=1)\end{array}$ \\
\hline \hline obstruktive Grunderkrankungen & $11,57 \pm 12,91$ Tage \\
& $(n=91)$ \\
\hline \hline restriktive Grunderkrankungen & $10,45 \pm 13,26$ Tage \\
$(n=22)$
\end{tabular}

Tabelle 3.1.2 zeigt die durchschnittliche stationäre Aufenthaltsdauer \pm Standardabweichung der 126 aufgenommenen Patienten untergliedert in vier Gruppen: keine definierte, obstruktive, restriktive und andere Grunderkrankungen. In Klammern ist die Anzahl der Patienten mit den jeweiligen Grunderkrankungen angegeben. 
Tabelle 3.1.3 listet die Gründe auf, welche zum Einleiten der Beatmung geführt haben.

Der gewählte Ausdruck „Grunderkrankung bedingt Beatmung“ beschreibt den Sachverhalt, dass durch die Grunderkrankung die Atmungspumpe überlastet wurde und ein zusätzliches Ereignis nicht erkennbar war. Z.B. kann dies im Rahmen einer COPD auftreten, wenn die Atmungsarbeit in dem Maße gesteigert werden muss, dass es zu einer Überbeanspruchung und demzufolge zur Erschöpfung des Atmungsapparates kommt. Die Grunderkrankung kann auch eine direkte Funktionsstörung der Atmungspumpe bewirken, wenn z.B. die Atmungsmuskulatur bei einer Muskeldystrophie in ihrer Funktion eingeschränkt ist oder die nervale Innervation der Atmungsmuskeln durch eine Spinale Muskelatrophie gestört ist.

\begin{tabular}{|l|c|}
\hline & $\begin{array}{c}\text { alle Fälle } \\
\text { (n=126) }\end{array}$ \\
\hline \hline Grunderkrankung bedingt Beatmung & $95(75,4 \%)$ \\
\hline \hline Infekt & $14(11,1 \%)$ \\
\hline Pneumothorax & $4(3,2 \%)$ \\
\hline \hline operativer Eingriff & $6(4,8 \%)$ \\
\hline davon Cholezystektomie & $1(0,8 \%)$ \\
\hline davon Entfernung supraglottische Zyste & $1(0,8 \%)$ \\
\hline davon Lungenbiopsie & $1(0,8 \%)$ \\
\hline davon nicht bekannt & $3(2,4 \%)$ \\
\hline \hline andere Ursachen & $7(5,6 \%)$ \\
\hline davon Reanimation & $3(2,4 \%)$ \\
\hline davon Intoxikation & $1(0,8 \%)$ \\
\hline davon Myokardinfarkt & $1(0,8 \%)$ \\
\hline davon Lungenembolie & $1(0,8 \%)$ \\
\hline davon medikamentenassoziierte allergische Alveolitis & $1(0,8 \%)$ \\
\hline
\end{tabular}

Tabelle 3.1.3 zeigt die Ursache der Beatmung der 126 aufgenommenen Patienten auf die RICU nach Einschätzung der behandelnden Ärzte. Angegeben ist die absolute Häufigkeit und in Klammern die relative Häufigkeit der jeweiligen Ursache. 


\subsection{Fälle mit Keimübertragungen}

Auf der RICU wurden während des Untersuchungszeitraums bei jedem Patienten regelmäßig mikrobiologische Proben abgenommen. 438 dieser Proben wiesen Erreger nach und insgesamt 667 Erreger wurden detektiert.

Bei 14 Patienten wurde eine Keimübertragung festgestellt. Bei den übrigen $112 \mathrm{~Pa}-$ tienten konnte eine Keimübertragung nicht nachgewiesen werden. Altersdurchschnitt und Geschlechtsverteilung beider Gruppen sind in Tabelle 3.2 aufgeführt.

\begin{tabular}{|l|c|c|c|}
\hline & $\begin{array}{c}\text { Fälle mit } \\
\text { Keimübertragungen } \\
(\mathbf{n = 1 4 )}\end{array}$ & $\begin{array}{c}\text { Fälle ohne } \\
\text { Keimübertragungen } \\
(\mathbf{n = 1 1 2})\end{array}$ & p-Wert \\
\hline Alter in Jahren & $66,07 \pm 12,43$ & $61,48 \pm 17,87$ & 0,184 \\
\hline Geschlecht $(\mathbf{m}: \mathbf{w})$ & $9: 5$ & $\begin{array}{c}74: 38 \\
(66,1 \%: 33,9 \%)\end{array}$ & 1 \\
\hline
\end{tabular}

Tabelle 3.2 zeigt das Alter und Geschlecht der 126 Fälle in den zwei Untergruppen: Fälle ohne und Fälle mit nachgewiesener Keimübertragung. Das Alter ist als Mittelwert \pm Standardabweichung angegeben. Signifikante Werte sind nicht zu verzeichnen.

Im Vergleich des Patientenalters zwischen der Patientengruppe mit und der Patientengruppe ohne Keimübertragung mittels des t-Tests bei unabhängigen Stichproben lag mit einem $p$-Wert von 0,184 kein signifikanter Unterschied vor.

Auch die Geschlechterverteilung war nicht signifikant unterschiedlich (nach

Fisher's exact test: $p=1$ ). 


\subsubsection{Grunderkrankungen}

Die Patienten zeigten die in Tabelle 3.2.1 genannten Grunderkrankungen auf.

\begin{tabular}{|c|c|c|c|}
\hline & $\begin{array}{c}\text { Fälle mit } \\
\text { Keimübertragungen } \\
(n=14)\end{array}$ & $\begin{array}{c}\text { Fälle ohne } \\
\text { Keimübertragungen } \\
(n=112)\end{array}$ & p-Wert \\
\hline $\begin{array}{l}\text { keine definierte } \\
\text { Grunderkrankung }\end{array}$ & $1(7,1 \%)$ & $0(0 \%)$ & 0,111 \\
\hline $\begin{array}{l}\text { obstruktive } \\
\text { Grunderkrankungen }\end{array}$ & $11(78,6 \%)$ & $80(71,4 \%)$ & 0,756 \\
\hline davon COPD & $11(78,6 \%)$ & $79(70,5 \%)$ & \\
\hline davon Asthma bronchiale & $0(0 \%)$ & $1(0,9 \%)$ & \\
\hline restriktive Grunderkrankungen & $1(7,1 \%)$ & $21(18,8 \%)$ & 0,461 \\
\hline davon ALS & $0(0 \%)$ & $4(3,6 \%)$ & \\
\hline $\begin{array}{l}\text { davon Muskeldystrophie } \\
\text { Duchenne }\end{array}$ & $1(7,1 \%)$ & $11(9,8 \%)$ & \\
\hline $\begin{array}{l}\text { davon andere restriktive Grun- } \\
\text { derkrankungen }\end{array}$ & $0(0 \%)$ & $6(5,4 \%)$ & \\
\hline andere Grunderkrankungen & $1(7,1 \%)$ & $11(9,8 \%)$ & 1,000 \\
\hline davon Lungenfibrose & $0(0 \%)$ & $3(2,7 \%)$ & \\
\hline davon Bronchialkarzinom & $0(0 \%)$ & $2(1,8 \%)$ & \\
\hline davon Herzinsuffizienz & $0(0 \%)$ & $2(1,8 \%)$ & \\
\hline $\begin{array}{l}\text { davon zentrale } \\
\text { Atemregulationsstörung }\end{array}$ & $1(7,1 \%)$ & $1(0,9 \%)$ & \\
\hline $\begin{array}{l}\text { davon andere } \\
\text { Grunderkrankungen }\end{array}$ & $0(0 \%)$ & $3(2,7 \%)$ & \\
\hline
\end{tabular}

Tabelle 3.2.1 zeigt die Grunderkrankungen der 126 aufgenommenen Patienten in den zwei Untergruppen: Fälle mit und Fälle ohne nachgewiesener Keimübertragung. Die Werte der Fälle mit und ohne Keimübertragungen wurden mittels Fisher's exact test verglichen und das Ergebnis als $p$-Wert angegeben. Signifikante Werte sind nicht zu verzeichnen.

Die Verteilung von nachgewiesenen Keimübertragungen auf die Grunderkrankung war in beiden Gruppen nicht signifikant unterschiedlich. Eine Keimübertragung fand primär bei an COPD erkrankten Patienten statt. Jedoch war die Anzahl der Übertragungen zu gering um hier einen statistischen Unterschied zu finden. 
Eine genaue Auflistung der Patienten mit restriktiven Grunderkrankungen sowie „anderen Grunderkrankungen“ in den zwei Untergruppen: Fälle mit und Fälle ohne nachgewiesener Keimübertragung, zeigt Tabelle 6.1 im Anhang.

\subsubsection{Ursache des Beatmungsbeginns}

Tabelle 3.2.2 listet die Gründe auf, welche zur Beatmung geführt haben.

\begin{tabular}{|c|c|c|c|}
\hline & $\begin{array}{c}\text { Fälle mit } \\
\text { Keimübertragungen } \\
(n=14)\end{array}$ & $\begin{array}{c}\text { Fälle ohne } \\
\text { Keimübertragungen } \\
(n=112)\end{array}$ & $\begin{array}{c}p- \\
\text { Wert }\end{array}$ \\
\hline $\begin{array}{l}\text { Grunderkrankung bedingt } \\
\text { Beatmung }\end{array}$ & $9(64,3 \%)$ & $86(68,3 \%)$ & 0,330 \\
\hline Infekt & $4(28,6 \%)$ & $10(8,9 \%)$ & 0,050 \\
\hline Pneumothorax & $1(7,1 \%)$ & $3(2,7 \%)$ & 0,380 \\
\hline Operativer Eingriff & $0(0 \%)$ & $6(5,4 \%)$ & 1,000 \\
\hline andere Ursachen & $0(0 \%)$ & $7(6,3 \%)$ & 1,000 \\
\hline davon Reanimation & $0(0 \%)$ & $3(2,7 \%)$ & \\
\hline davon Intoxikation & $0(0 \%)$ & $1(0.9 \%)$ & \\
\hline davon Myokardinfarkt & $0(0 \%)$ & $1(0.9 \%)$ & \\
\hline davon Lungenembolie & $0(0 \%)$ & $1(0.9 \%)$ & \\
\hline $\begin{array}{l}\text { davon medikamentenassoziierte } \\
\text { allergische Alveolitis }\end{array}$ & $0(0 \%)$ & $1(0.9 \%)$ & \\
\hline
\end{tabular}

Tabelle 3.2.2 zeigt die Ursache der Beatmung nach Einschätzung der behandelnden Ärzte der 126 aufgenommenen Patienten in den zwei Untergruppen: Fälle ohne und Fälle mit nachgewiesener Keimübertragung. Die Werte der Fälle mit und ohne Keimübertragungen wurden mittels Fisher's exact test verglichen und das Ergebnis als $p$-Wert angegeben. Signifikante Werte sind nicht zu verzeichnen.

Die Verteilung der Beatmungsursache war in beiden Gruppen nicht signifikant unterschiedlich: nach Fisher's exact test waren alle $p$-Werte größer als fünf Prozent.

Die einzelnen operativen Eingriffe sind in Tabelle 3.1.3 aufgelistet (siehe Abschnitt 3.1: "Grunderkrankungen und Ursachen der Beatmung"). 


\subsubsection{Liegedauer}

Tabelle 3.2.3 stellt die durchschnittlichen, die minimalen und maximalen Aufenthaltsdauern mit deren Standardabweichungen der Patienten auf der RICU dar. Die Gesamtaufenthaltsdauer wurde in Quartilen (25\%-, 50\%- und 75\%-Quartil) dargestellt.

\begin{tabular}{|c|c|c|c|}
\hline & & $\begin{array}{c}\text { Fälle mit } \\
\text { Keimübertragungen } \\
(n=14)\end{array}$ & $\begin{array}{c}\text { Fälle ohne } \\
\text { Keimübertragungen } \\
(n=112)\end{array}$ \\
\hline \multirow{2}{*}{\multicolumn{2}{|c|}{$\begin{array}{l}\text { Mittelwert } \pm \text { Standardabweichung der } \\
\text { stationären Aufenthaltsdauer eines } \\
\text { Falls }\end{array}$}} & $24,07 \pm 23,84$ Tage & $9,87 \pm 10,79$ Tage \\
\hline & & \multicolumn{2}{|c|}{$p-$ Wert $=0,013$} \\
\hline \multicolumn{2}{|c|}{$\begin{array}{l}\text { Minimum und Maximum der } \\
\text { Aufenthaltsdauer }\end{array}$} & 1 und 79 Tage & 1 und 54 Tage \\
\hline \multirow{3}{*}{$\begin{array}{l}\text { Aufenthaltsdauer } \\
\text { in Quartilen }\end{array}$} & 1. Quartil & 7,25 Tage & 3,00 Tage \\
\hline & 2. Quartil & 16,50 Tage & 7,00 Tage \\
\hline & 3. Quartil & 46,75 Tage & 13,00 Tage \\
\hline
\end{tabular}

Tabelle 3.2.3 zeigt die durchschnittliche stationäre Aufenthaltsdauer \pm Standardabweichung, die minimale und maximale Aufenthaltsdauer und die Aufenthaltsdauern in Quartilen angegeben der 126 auf der Intensivstation befindlichen Patienten in den zwei Untergruppen: Fälle ohne und Fälle mit nachgewiesener Keimübertragung. Die stationären Aufenthaltsdauern der Fälle mit und ohne Keimübertragungen wurden mittels Mann-Whitney-U-Test verglichen und das Ergebnis als $p$-Wert angegeben. Ein signifikanter Wert ist zu verzeichnen.

Die Dauer des stationären Aufenthalts der Patienten mit und der Patienten ohne Keimübertragung unterschieden sich signifikant (im Mann-Whitney-U-Test: $p=0,013 ; \alpha<=0,05)$.

Aufgrund der hohen Standardabweichung wurde die Aufenthaltsdauer der Patienten mit dem kürzesten und längsten stationären Aufenthalt, sowie die Aufenthaltsdauer der Patienten untergliedert in Quartile angegeben. Die minimale Aufenthaltsdauer in den zwei Gruppen betrug einen Tag. Der Patient mit dem längsten Aufenthalt, welcher 79 Tage betrug, ist zum Kollektiv mit nachgewiesener Keimübertragung zu zählen.

Anhand der Quartile ist zu erkennen, dass in der Gruppe mit Keimübertragungen $25 \%$ der Patienten weniger als 7,25 Tage, 50\% weniger als 16,50 Tage und 75\% weniger als 46,75 Tage stationär auf der RICU behandelt wurden. Im Kollektiv der 
Patienten ohne diagnostizierte Keimübertragung lagen $25 \%$ der Patienten weniger als 3 Tage, 50\% weniger als 16,50 Tage und 75\% weniger als 46,75 Tage auf der RICU. Die Unterteilung in Quartile zeigt, dass die Verteilungen der Aufenthaltsdauern der Patienten mit und ohne Keimübertragung vergleichbar sind.

\subsubsection{Anzahl der Patientenkontakte und gemeinsame Liegedauer der Patientenkontakte}

Im Jahre 2010 konnten auf der RICU insgesamt 363 unterschiedliche Patientenpaare, also jeweils zwei Patienten, die sich zeitgleich auf der RICU befanden, gezählt werden. Die durchschnittliche gemeinsame Liegedauer eines Patientenpaares betrug 6,31 Tage (Standardabweichung $\pm 6,10$ Tage). Die Patientenpaare, zwischen denen Keimübertragungen stattgefunden haben bzw. nicht stattgefunden haben, werden in der Tabelle 3.2.4 als „Patientenkontakte“ angegeben. Zusätzlich werden die durchschnittliche gemeinsame Liegedauer mit Standardabweichung, die minimale und maximale Aufenthaltsdauer und eine Einteilung in Quartile aufgelistet.

\begin{tabular}{|c|c|c|c|}
\hline & & $\begin{array}{l}\text { Patientenpaare mit } \\
\text { Keimübertragung }\end{array}$ & $\begin{array}{l}\text { Patientenpaare ohne } \\
\text { Keimübertragung }\end{array}$ \\
\hline \multicolumn{2}{|c|}{ Anzahl der Patientenkontakte } & 11 & 352 \\
\hline \multirow{2}{*}{\multicolumn{2}{|c|}{$\begin{array}{l}\text { Mittelwert } \pm \text { Standardabweichung der } \\
\text { gemeinsamen Liegedauer der } \\
\text { Patientenkontakte }\end{array}$}} & $8,6 \pm 7,78$ Tage & $6,2 \pm 6,04$ Tage \\
\hline & & \multicolumn{2}{|c|}{$\mathrm{p}$-Wert $=0,310$} \\
\hline \multicolumn{2}{|c|}{$\begin{array}{l}\text { Minimum und Maximum der } \\
\text { gemeinsamen Liegedauer der } \\
\text { Patientenkontakte }\end{array}$} & 1 und 26 Tage & 1 und 40 Tage \\
\hline \multirow{3}{*}{$\begin{array}{l}\text { Aufenthaltsdauer } \\
\text { in Quartilen }\end{array}$} & 1. Quartil & 2 Tage & 2 Tage \\
\hline & 2. Quartil & 6 Tage & 4 Tage \\
\hline & 3. Quartil & 13 Tage & 8 Tage \\
\hline
\end{tabular}

Tabelle 3.2.4 zeigt die Anzahl der Patientenkontakte, Mittelwert \pm Standardabweichung der gemeinsamen Liegedauer der Patientenkontakte, die minimale und maximale gemeinsame Liegedauer und die Aufenthaltsdauern in Quartilen der 363 auf der Intensivstation befindlichen Patientenpaare in den zwei Untergruppen: Patientenpaare mit und Patientenpaare ohne nachgewiesene Keimübertragung. Die gemeinsame Liegedauer der Fälle mit und ohne Keimübertragungen wurden mittels Mann-Whitney-U-Test verglichen und das Ergebnis als $\mathrm{p}$-Wert angegeben. Ein signifikanter Wert ist nicht zu verzeichnen. 
Die durchschnittliche gemeinsame Liegedauer der Patientenkontakte, bei denen eine Keimübertragung nachgewiesen wurde und bei denen keine Keimübertragung nachgewiesen wurde, erwies sich als nicht signifikant unterschiedlich (im MannWhitney-U-Test: $p=0,310 ; \alpha<=0,05$ ).

Wie bei der stationären Aufenthaltsdauer lag auch hier eine hohe Standardabweichung vor. Daher wurden die Patientenpaare mit der kürzesten und längsten gemeinsamen Liegedauer angegeben.

Anhand der Berechnung der Quartile ist zu erkennen, dass in der Gruppe mit Keimübertragungen 25\% der Patientenpaare weniger als 2 Tage, 50\% weniger als 6 Tage und $75 \%$ weniger als 13 Tage stationär auf der RICU behandelt wurden. Im Kollektiv der Patientenpaare ohne diagnostizierter Keimübertragung waren 25\% der Paare weniger als 2 Tage, $50 \%$ weniger als 4 Tage und $75 \%$ weniger als 8 Tage gemeinsam auf der RICU. Auch hier zeigt die Unterteilung in Quartile, dass die Verteilungen der gemeinsamen Liegedauer der Patienten mit und ohne Keimübertragung vergleichbar sind. 


\subsubsection{Therapie bei Entlassung}

87 der 126 Patienten wurden mit einer ISB-Therapie über einen nicht-invasiven Zugang mittels volumen- bzw. druckkontrollierter Beatmung (VCV bzw. PCV) entlassen. Bei fünf Patienten musste die ISB-Therapie über einen invasiven Zugang stattfinden. Einem Patienten wurde Sauerstoff ohne jegliche Beatmungstherapie appliziert.

Tabelle 3.2.5 listet die Therapieformen der Patienten mit versus ohne Keimübertragungen bei deren Entlassung auf. Zur Vollständigkeit werden die 14 Patienten mit aufgeführt, die während ihres Aufenthalts auf der Intensivstation verstarben.

\begin{tabular}{|l|c|c|c|}
\hline Therapie bei Entlassung & $\begin{array}{c}\text { Fälle mit } \\
\text { Keimübertragungen } \\
(\mathbf{n = 1 4 )}\end{array}$ & $\begin{array}{c}\text { Fälle ohne } \\
\text { Keimübertragungen } \\
(\mathbf{n = 1 1 2})\end{array}$ & p-Wert \\
\hline $\begin{array}{l}\text { ISB-Therapie über nicht-inva- } \\
\text { siven Zugang (Maske) VCV } \\
\text { bzw. PCV }\end{array}$ & $7(50 \%)$ & $80(71,4 \%)$ & 0,128 \\
\hline $\begin{array}{l}\text { ISB-Therapie über invasiven } \\
\text { Zugang (Tracheostoma) }\end{array}$ & $3(21,4 \%)$ & $2(1,8 \%)$ & 0,010 \\
\hline $\begin{array}{l}\text { keine Beatmung } \\
\text { mit O2-Gabe }\end{array}$ & $0(0 \%)$ & $1(0,9 \%)$ & 1,000 \\
\hline $\begin{array}{l}\text { keine Beatmung } \\
\text { ohne O2-Gabe }\end{array}$ & $3(7,1 \%)$ & $18(16,1 \%)$ & 0,692 \\
\hline davon verstorben & $3(21,4 \%)$ & $11(9,8 \%)$ & 0,189 \\
\hline
\end{tabular}

Tabelle 3.2.5 zeigt die Therapie bei Entlassung der 126 aufgenommenen Patienten in den zwei Untergruppen: Fälle ohne und Fälle mit nachgewiesener Keimübertragung. Die Werte der Fälle ohne und mit Keimübertragungen wurden mittels Fisher's exact test verglichen und das Ergebnis als $p$ Wert angegeben. Ein signifikanter Wert ist zu verzeichnen.

Es wurden in der Gruppe mit Keimübertragungen signifikant mehr invasiv beatmete Patienten entlassen.

\subsubsection{Mortalität und aufgetretene Infekte}

Die 90-Tage-Mortalität betrug mit 28 Patienten 22\%. 14 Patienten und somit 11\% der gesamten Fälle verstarben schon während ihres stationären Aufenthalts auf der RICU, 14 weitere innerhalb 90 Tagen nach Entlassung. 
Die Mortalität der Patienten, die während des stationären Aufenthalts und die bis 90 Tage nach ihrer Entlassung verstarben, sowie die Anzahl der Fälle mit aufgetretenen Infekten und deren 90-Tage-Mortalität sind in Tabelle 3.2.6 für Patienten ohne und mit aufgetretener Keimübertragung aufgelistet.

\begin{tabular}{|c|c|c|c|}
\hline & $\begin{array}{c}\text { Fälle mit } \\
\text { Keimübertragungen } \\
(n=14)\end{array}$ & $\begin{array}{c}\text { Fälle ohne } \\
\text { Keimübertragungen } \\
(n=112)\end{array}$ & p-Wert \\
\hline $\begin{array}{l}\text { davon stationär } \\
\text { verstorben }\end{array}$ & $3(21,4 \%)$ & $11(9,8 \%)$ & 0,189 \\
\hline 90-Tage-Mortalität & $7(50 \%)$ & $21(18,8 \%)$ & 0,015 \\
\hline \multirow[t]{2}{*}{$\begin{array}{l}\text { Anzahl der Fälle mit } \\
\text { aufgetretenen Infekten }\end{array}$} & $3(21,4 \%)$ & $20(17,9 \%)$ & 0,719 \\
\hline & 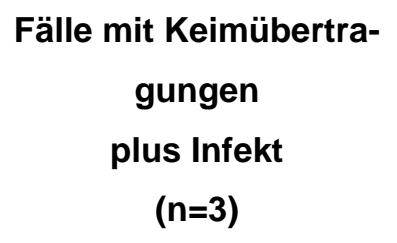 & $\begin{array}{l}\text { Fälle ohne Keimüber- } \\
\text { tragungen } \\
\text { plus Infekt } \\
(n=20)\end{array}$ & 0,571 \\
\hline 90-Tage-Mortalität & $1(33,3 \%)$ & $6(30 \%)$ & 1,000 \\
\hline
\end{tabular}

Tabelle 3.2.6 zeigt die Anzahl der Patienten, die während ihres stationären Aufenthalts verstarben, die 90-Tage-Mortalität (Tod innerhalb 90 Tage nach Entlassung) der 126 aufgenommenen Patienten sowie die Anzahl der Patienten mit aufgetretenen Infekten und die 90-Tage-Mortalität bei den Patienten mit diagnostizierter Infektion in den zwei Untergruppen: Fälle ohne und Fälle mit nachgewiesener Keimübertragung. Die Werte der Fälle ohne und mit Keimübertragungen wurden mittels Fisher's exact test verglichen und das Ergebnis als p-Wert angegeben. Ein signifikanter Wert ist zu verzeichnen.

Die 90-Tage-Mortalität der Patienten mit Keimübertragungen war signifikant höher als die ohne Keimübertragungen (nach Fisher's exact test: $p=0,0146 ; \alpha<=0,05$ ). Auch die Sterblichkeit der Patienten mit Keimübertragung während des stationären Aufenthalts war höher, jedoch nicht signifikant (nach Fisher's exact test: $p=0,1888$; $\alpha<=0,05)$.

Zwischen der Anzahl der Patienten mit Infektionen und aufgetretener Keimübertragung und der Anzahl der Patienten mit Infektion ohne aufgetretener Keimübertragung bestand kein signifikanter Unterschied (nach Fisher's exact test: $p=0,7193$; $\alpha<=0,05)$.

Es war kein signifikanter Unterschied festzustellen zwischen der Mortalität der Patienten mit aufgetretenen Infekten sowie stattgefundener Keimübertragung und der 
Mortalität der Patienten mit aufgetretenen Infekten, aber ohne Keimübertragung (nach Fisher's exact test: $p=0,5709 ; \alpha<=0,05$ ). 


\subsubsection{Blutgase unter Spontanatmung}

Die Blutgase der auf der RICU stationär behandelten 126 Patienten wurden bei deren Aufnahme und Entlassung bestimmt. In den folgenden Tabellen sind sie auf zwei unterschiedliche Weisen verglichen worden, zum einen bei Patienten mit versus ohne Keimübertragungen (siehe Tabellen 3.2.7.1.a und b) und zum anderen bei Aufnahme versus Entlassung (siehe Tabellen 3.2.7.1.a und b).

\subsubsection{Blutgase bei Fällen mit versus ohne Keimübertragungen}

\begin{tabular}{|l|c|c|c|c|}
\hline \multicolumn{5}{|c|}{ Bei Aufnahme } \\
\hline $\begin{array}{l}\text { mit Keimübertragungen } \\
(\mathbf{n = 1 2})\end{array}$ & $\mathbf{p H}$ & $\mathbf{p C O}_{2}$ in $\mathbf{~ m m H g}$ & $\mathbf{p O}_{2}$ in $\mathbf{~ m m H g}$ & $\mathrm{HCO}_{3}$ - in $\mathbf{~ m m o l} / \mathbf{l}$ \\
\hline $\begin{array}{l}\text { ohne Keimübertragungen } \\
(\mathbf{n = 1 0 5 )}\end{array}$ & $7,43 \pm 0,094$ & $53,30 \pm 14,73$ & $70,72 \pm 13,24$ & $32,97 \pm 6,56$ \\
\hline p-Wert im t-Test & 0,607 & 0,732 & 0,224 & 0,388 \\
\hline
\end{tabular}

Tabelle 3.2.7.1.a zeigt die Blutgase der Patienten bei deren Aufnahme. Die Werte sind als Mittelwert \pm Standardabweichung angegeben. In Klammern steht die Anzahl der Patienten, bei denen die Werte bekannt waren. Die Werte der Fälle ohne und mit Keimübertragungen wurden im t-Test für unverbundene Stichproben verglichen und das Ergebnis als p-Wert angegeben. Ein signifikanter Wert ist nicht zu verzeichnen.

\begin{tabular}{|l|c|c|c|c|}
\hline \multicolumn{5}{|c|}{ Bei Entlassung } \\
\hline & $\mathbf{p H}$ & $\mathrm{pCO}_{2}$ in $\mathbf{~ m m H g}$ & $\mathbf{p O}_{2}$ in $\mathbf{~ m m H g}$ & $\mathbf{H C O}_{3}$ - in mmol/I \\
\hline $\begin{array}{l}\text { mit Keimübertragung } \\
(\mathbf{n = 1 0})\end{array}$ & $7,42 \pm 0,063$ & $52,46 \pm 12,76$ & $61,76 \pm 11,00$ & $31,16 \pm 5,17$ \\
\hline $\begin{array}{l}\text { ohne Keimübertragung } \\
(\mathbf{n}=\mathbf{8 3})\end{array}$ & $7,42 \pm 0,059$ & $48,46 \pm 9,61$ & $66,71 \pm 15,61$ & $29,50 \pm 4,84$ \\
\hline $\begin{array}{l}\text { p-Wert im t-Test bei } \\
\text { unabhängigen Stichproben }\end{array}$ & 0,730 & 0,307 & 0,093 & 0,628 \\
\hline
\end{tabular}

Tabelle 3.2.7.1.b zeigt die Blutgase der Patienten bei deren Entlassung. Die Werte sind als Mittelwert \pm Standardabweichung angegeben. In Klammern steht die Anzahl der Patienten, bei denen die Werte bekannt waren. Die Werte der Fälle ohne und mit Keimübertragungen wurden im t-Test für unverbundene Stichproben verglichen und das Ergebnis als p-Wert angegeben. Ein signifikanter Wert ist nicht zu verzeichnen.

Vergleicht man die Blutgase der Patienten mit versus ohne diagnostizierte Keimübertragung jeweils bei deren Aufnahme und Entlassung miteinander, so ergeben sich zwischen diesen keine statistisch signifikanten Unterschiede. 


\subsubsection{Blutgase bei Aufnahme versus Entlassung}

\begin{tabular}{|l|c|c|c|c|}
\hline \multicolumn{5}{|c|}{ Fälle mit Keimübertragungen $(\mathbf{n = 1 0})$} \\
\hline & $\mathbf{p H}$ & $\mathbf{p C O}_{2}$ in $\mathbf{~ m m H g}$ & $\mathbf{p O}_{2}$ in $\mathbf{~ m m H g}$ & $\mathbf{H C O}_{3^{-}}$in $\mathbf{~ m o l} / \mathbf{l}$ \\
\hline bei Aufnahme & $7,43 \pm 0,05$ & $56,79 \pm 13,56$ & $68,65 \pm 12,31$ & $32,33 \pm 5,99$ \\
\hline bei Entlassung & $7,42 \pm 0,06$ & $52,46 \pm 12,76$ & $61,76 \pm 11,00$ & $31,16 \pm 5,17$ \\
\hline p-Wert im t-Test & 0,382 & 0,128 & 0,165 & 0,615 \\
\hline
\end{tabular}

Tabelle 3.2.7.2.a zeigt die Blutgase der 10 Fälle mit Keimübertragung bei Aufnahme und Entlassung, bei denen die gepaarten Werte bekannt waren. Die Werte sind als Mittelwert \pm Standardabweichung angegeben. Die Werte bei Aufnahme und Entlassung wurden im t-Test für verbundene Stichproben verglichen und das Ergebnis als $p$-Wert angegeben. Ein signifikanter Wert ist nicht zu verzeichnen.

\begin{tabular}{|l|c|c|c|c|}
\hline \multicolumn{5}{|c|}{ Fälle ohne Keimübertragungen $(\mathbf{n = 8 0})$} \\
\hline bei Aufnahme & $\mathbf{p H}$ & $\mathrm{pCO}_{2}$ in $\mathbf{~ m m H g}$ & $\mathrm{pO}_{2}$ in $\mathbf{~ m m H g}$ & $\mathbf{H C O}_{3}$ - in $\mathbf{~ m o l} / \mathbf{l}$ \\
\hline bei Entlassung & $7,40 \pm 0,08$ & $53,83 \pm 14,40$ & $69,48 \pm 25,11$ & $30,78 \pm 5,88$ \\
\hline \hline p-Wert im t-Test & $7,42 \pm 0,06$ & $48,29 \pm 9,72$ & $67,10 \pm 15,76$ & $29,54 \pm 4,91$ \\
\hline
\end{tabular}

Tabelle 3.2.7.2.b zeigt die Blutgase der 80 Fälle ohne Keimübertragung bei Aufnahme und Entlassung, bei denen die gepaarten Werte bekannt waren. Die Werte sind als Mittelwert \pm Standardabweichung angegeben. Die Werte bei Aufnahme und Entlassung wurden im t-Test für verbundene Stichproben verglichen und das Ergebnis als $p$-Wert angegeben. Ein signifikanter Wert ist zu verzeichnen.

Vergleicht man die Blutgase der Patienten bei deren Aufnahme und Entlassung miteinander, so ergeben sich zwischen diesen keine statistisch signifikanten Unterschiede. Eine Ausnahme stellen der $\mathrm{PCO}_{2}$ - und $\mathrm{HCO}_{3}-W e r t$ bei den Patienten ohne Keimübertragungen dar: Der p-Wert im t-Test beträgt 0,000154 bzw. 0,033 und ist somit statistisch signifikant. 


\subsubsection{Lungenfunktion}

In Tabelle 3.2.8.a sind die Lungenfunktionen der Patienten mit versus ohne Keimübertragungen bei den Patienten mit obstruktiver Grunderkrankung aufgezeigt. Tabelle 3.2.8.b listet dies bei den Patienten mit restriktiver Grunderkrankung auf.

\begin{tabular}{|c|c|c|c|c|c|}
\hline \multicolumn{6}{|c|}{$\begin{array}{l}\text { Fälle mit obstruktiver Grunderkrankung } \\
\qquad(\mathrm{n}=91)\end{array}$} \\
\hline \multirow[b]{2}{*}{ Alter in Jahren } & \multicolumn{2}{|c|}{$\begin{array}{c}\text { mit Keimübertragungen } \\
(n=11)\end{array}$} & \multicolumn{2}{|c|}{$\begin{array}{l}\text { ohne Keimübertragungen } \\
(\mathrm{n}=80)\end{array}$} & \multirow{2}{*}{$\frac{\mathbf{p} \text {-Wert im t-Test }}{0,283}$} \\
\hline & $69,09 \pm 9,34$ & $(n=11)$ & $68,05 \pm 10,173$ & $(n=80)$ & \\
\hline TLC (I) & 6,63 & $(n=3)$ & 6,11 & $(n=75)$ & 0,719 \\
\hline IVC (I) & $1,64 \pm 0,56$ & $(n=2)$ & $1,86 \pm, 54$ & $(n=61)$ & 0,850 \\
\hline IVC (\%) & $48,85 \pm 0,35$ & $(n=2)$ & $51,90 \pm 13,60$ & $(n=60)$ & 0,040 \\
\hline FEV1 (I) & $0,88 \pm 0,43$ & $(n=2)$ & $1,02 \pm 0,39$ & $(n=60)$ & 0,980 \\
\hline FEV1 (\%) & $38,15 \pm 29,20$ & $(n=2)$ & $37,76 \pm 13,92$ & $(n=60)$ & 0,096 \\
\hline FEV1/IVC (\%) & $63,07 \pm 47,64$ & $(n=2)$ & $54,71 \pm 15,64$ & $(n=60)$ & 0,002 \\
\hline$R$ tot $\left(\mathrm{kPa}^{*} \mathrm{~s} / \mathrm{l}\right)$ & $0,80 \pm 0,51$ & $(n=2)$ & $0,88 \pm, 72$ & $(n=59)$ & 0,847 \\
\hline RV/TLC (\%) & $67,56 \pm 8,06$ & $(n=2)$ & $68,81 \pm 13,77$ & $(n=59)$ & 0,568 \\
\hline
\end{tabular}

Tabelle 3.2.8.a zeigt das Alter und die Lungenfunktion der 91 aufgenommenen Patienten mit obstruktiver Grunderkrankung in den zwei Untergruppen: Fälle ohne und Fälle mit nachgewiesener Keimübertragung. Die Werte sind als Mittelwert \pm Standardabweichung angegeben und in Klammern die Anzahl der Patienten, bei denen die Untersuchung durchgeführt wurde. Die Werte der Fälle ohne und mit Keimübertragungen wurden im t-Test für unverbundene Stichproben verglichen und das Ergebnis als $p$-Wert angegeben. Signifikante Werte sind zu verzeichnen.

Vergleicht man die Lungenfunktion der Patienten ohne und mit Keimübertragungen, zeigten die Patienten mit Keimübertragung eine signifikant niedrigere IVC (\%) mit $p=0,04$, aber eine signifikant höhere FEV1/IVC(\%) mit $p=0,002$. Ihre Erkrankung war also weit fortgeschritten.

Bei den Fällen mit einer restriktiven Grunderkrankung kam es nur zu einer Keimübertragung. Da dieser Patient stationär verstarb, konnten keine Lungenfunktionsuntersuchungen bei Entlassung durchgeführt werden und somit die Lungenfunktion nicht verglichen werden. Zur Vollständigkeit und Beschreibung der Schwere der Lungenerkrankung bei den Fällen mit restriktiver Grunderkrankung wurde die folgende Tabelle mit aufgeführt. 


\begin{tabular}{|c|c|c|c|c|}
\hline \multicolumn{5}{|c|}{$\begin{array}{l}\text { Fälle mit restriktiver Grunderkrankung } \\
\qquad(\mathrm{n}=22)\end{array}$} \\
\hline \multirow[b]{2}{*}{ Alter in Jahren } & \multicolumn{2}{|c|}{$\begin{array}{l}\text { mit Keimübertragungen } \\
(n=1)\end{array}$} & \multicolumn{2}{|c|}{$\begin{array}{l}\text { ohne Keimübertragungen } \\
\qquad(n=21)\end{array}$} \\
\hline & $43 \pm 0$ & $(n=1)$ & $36,86 \pm 19,89$ & $(n=21)$ \\
\hline IVC (I) & - & $(n=1)$ & $1,21 \pm, 90$ & $(n=14)$ \\
\hline IVC (\%) & - & $(n=1)$ & $29,38 \pm 22,17$ & $(n=14)$ \\
\hline FEV1 (I) & - & $(n=1)$ & $1,08 \pm, 73$ & $(n=14)$ \\
\hline FEV1 (\%) & - & $(n=1)$ & $31,76 \pm 22,42$ & $(n=14)$ \\
\hline FEV1/IVC (\%) & - & $(n=1)$ & $89,41 \pm 7,86$ & $(n=14)$ \\
\hline $\mathrm{R}$ tot $\left(\mathrm{kPa}^{*} \mathrm{~s} / \mathrm{l}\right)$ & - & $(n=1)$ & $0,37 \pm, 69$ & $(n=11)$ \\
\hline RV/TLC (\%) & - & $(n=1)$ & $57,93 \pm 27,58$ & $(n=11)$ \\
\hline
\end{tabular}

Tabelle 3.2.8.b zeigt das Alter und die Lungenfunktion der 22 aufgenommenen Patienten mit restriktiver Grunderkrankung in den zwei Untergruppen: Fälle ohne und Fälle mit nachgewiesener Keimübertragung. Die Werte sind als Mittelwert \pm Standardabweichung angegeben und in Klammern die Anzahl der Patienten, bei denen die Untersuchung durchgeführt wurde. 


\section{3 Übertragene Bakterienstämme}

Die folgenden sechs Bakterienstämme, die im Jahre 2010 auf der RICU von einem zum anderen Patienten übertragen wurden, konnten nachgewiesen werden:

- zwei verschiedene Staphylococcus aureus-Stämme (davon ein MRSA)

- ein Pseudomonas-Stamm

- ein Enterococcus ssp.-Stamm

- ein Enterococcus faecium-Stamm

- ein Escherichia coli-Stamm.

Der Enterococcus ssp.-Stamm wurde dreimal, der Escherichia coli-Stamm zweimal und die übrigen Bakterienstämme jeweils einmal übertragen.

Eine genaue Auflistung der Patientenpaare, bei denen eine Keimübertragung stattgefunden hat, ist in Tabelle 6.2 mit Liegedauer auf der RICU und übertragenen Keimen im Anhang dargestellt. 


\subsection{Fälle ohne und mit Infektionen}

Während des Untersuchungszeitraums diagnostizierte der jeweils behandelnde Arzt bei 23 Patienten mindestens eine Infektion. Bei 103 Patienten trat keine Infektion auf. Grafik 3.4 zeigt die aufgetretenen Infektionen auf:

\section{Verteilung der 33 aufgetretenen Infektionen}

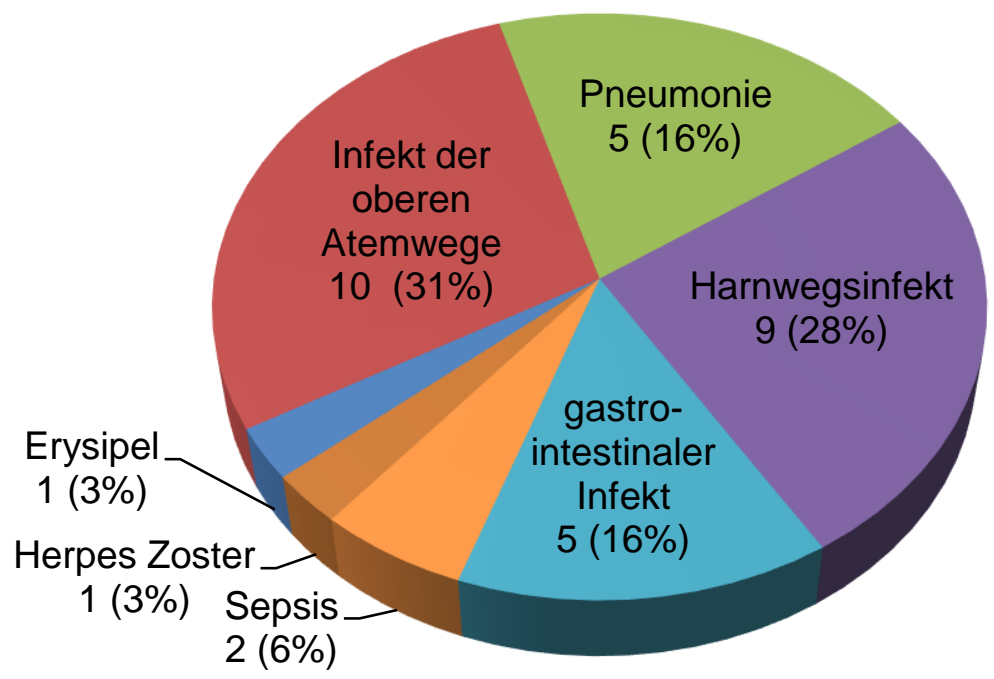

Grafik 3.4 zeigt die Art, die Anzahl und die relative Häufigkeit in Prozent der 33 aufgetretenen Infektionen bei den 126 auf der RICU stationären Patienten.

Alter, Geschlecht und Liegedauer der Patienten sind in Tabellen 3.4 aufgeführt.

\begin{tabular}{|l|c|c|c|}
\hline & $\begin{array}{c}\text { Fälle ohne Infektion } \\
(\mathbf{n = 1 0 3} \bumpeq 82 \%)\end{array}$ & $\begin{array}{c}\text { Fälle mit Infektion } \\
(\mathbf{n = 2 3} \bumpeq 18 \%)\end{array}$ & p-Wert \\
\hline Alter in Jahren & $61,05 \pm 18,23$ & $66,22 \pm 12,24$ & 0,055 \\
\hline $\begin{array}{l}\text { Geschlecht } \\
(\mathbf{m}: \mathbf{w})\end{array}$ & $\begin{array}{c}70: 33 \\
(68 \%: 32 \%)\end{array}$ & $\begin{array}{c}13: 10 \\
(56,5 \%: 43,5 \%)\end{array}$ & 0,3348 \\
\hline Liegedauer & $11,26 \pm 13,84$ & $12,26 \pm 12,19$ & 0,438 \\
\hline
\end{tabular}

Tabelle 3.4 zeigt das Alter, das Geschlecht und die Liegedauer der 126 aufgenommenen Patienten in zwei Gruppen: Fälle ohne und Fälle mit aufgetretener Infektion. Das Alter und die Liegedauer sind als Mittelwert \pm Standardabweichung angegeben. Signifikante Werte sind nicht zu verzeichnen.

Zwischen dem Patientenalter der Patienten ohne und mit Infektion lag im t-Test bei unabhängigen Stichproben mit einem $p$-Wert von 0,055 kein signifikanter Unterschied vor. 
Auch nicht signifikant unterschiedlich waren die Geschlechterverteilung (nach Fisher's exact test: $p=0,3348$ ) und die Liegedauer der Patientengruppen (im MannWhitney-U-Test: $p=0,438 ; \alpha<=0,05)$.

\subsubsection{Grunderkrankungen}

Die in Tabelle 3.4.1 aufgeführten Grunderkrankungen wurden bei den Patienten diagnostiziert.

\begin{tabular}{|c|c|c|c|}
\hline & $\begin{array}{l}\text { Fälle ohne Infektion } \\
\qquad(n=103)\end{array}$ & $\begin{array}{l}\text { Fälle mit Infektion } \\
\qquad(n=23)\end{array}$ & $\begin{array}{c}p- \\
\text { Wert }\end{array}$ \\
\hline keine definierte Grunderkrankung & $1(1 \%)$ & $0(0 \%)$ & 1,000 \\
\hline obstruktive Grunderkrankungen & $72(69,9 \%)$ & $19(82,6 \%)$ & 0,305 \\
\hline davon COPD & $71(68,9 \%)$ & $19(82,6 \%)$ & \\
\hline davon Asthma bronchiale & $1(1 \%)$ & $0(0 \%)$ & \\
\hline restriktive Grunderkrankungen & $22(21,4 \%)$ & $0(0 \%)$ & 0,013 \\
\hline davon ALS & $4(3,9 \%)$ & $0(0 \%)$ & \\
\hline davon Muskeldystrophie Duchenne & $12(11,7 \%)$ & $0(0 \%)$ & \\
\hline $\begin{array}{l}\text { davon andere restriktive Grunder- } \\
\text { krankungen }\end{array}$ & $6(5,8 \%)$ & $0(0 \%)$ & \\
\hline andere Grunderkrankungen & $8(7,8 \%)$ & $4(17,4 \%)$ & 0,230 \\
\hline
\end{tabular}

Tabelle 3.4.1 zeigt die Grunderkrankungen der 126 aufgenommenen Patienten in zwei Gruppen: Fälle ohne und Fälle mit aufgetretener Infektion. Die Werte der Fälle ohne und mit Infektionen wurden mittels Fisher's exact test verglichen und das Ergebnis als $p$-Wert angegeben. Das Signifikanzniveau wurde nach Bonferroni bei multiplen Testen korrigiert: $\alpha \leq 0,0125$. Signifikante Werte sind nicht zu verzeichnen.

Zwischen den Patientengruppen ohne und mit nachgewiesener Infektion konnte weder bei restriktiver noch bei obstruktiver Grunderkrankung (noch bei einer anderen) ein signifikanter Unterschied im Fisher's exact test mit Bonferroni-Korrektur festgestellt werden.

Eine genaue Auflistung der Patienten mit restriktiven Grunderkrankungen sowie „anderen“ Erkrankungen zeigt Tabelle 6.3 im Anhang. 


\subsubsection{Ursache des Beatmungsbeginns}

Tabelle 3.4.2 listet die Gründe auf, welche zur Beatmung geführt haben.

\begin{tabular}{|c|c|c|c|}
\hline & $\begin{array}{l}\text { Fälle ohne Infektion } \\
\qquad(n=103)\end{array}$ & $\begin{array}{l}\text { Fälle mit Infektion } \\
\qquad(n=23)\end{array}$ & p-Wert \\
\hline $\begin{array}{l}\text { Erschöpfung der Atmungspumpe } \\
\text { im Rahmen der Grunderkrankung }\end{array}$ & $76(73,8 \%)$ & $19(82,6 \%)$ & 0,436 \\
\hline infektassoziiert & $11(10,7 \%)$ & $3(13 \%)$ & 0,719 \\
\hline Pneumothorax & $4(3,9 \%)$ & $0(0 \%)$ & 1,000 \\
\hline operativer Eingriff & $6(5,8 \%)$ & $0(0 \%)$ & 0,591 \\
\hline andere Ursache & $6(5,8 \%)$ & $1(4,3 \%)$ & 1,000 \\
\hline davon Reanimation & $2(1,9 \%)$ & $1(4,3 \%)$ & \\
\hline davon Intoxikation & $1(1 \%)$ & $0(0 \%)$ & \\
\hline davon Myokardinfarkt & $1(1 \%)$ & $0(0 \%)$ & \\
\hline davon Lungenembolie & $1(1 \%)$ & $0(0 \%)$ & \\
\hline $\begin{array}{l}\text { davon medikamentenassoziierte al- } \\
\text { lergische Alveolitis }\end{array}$ & $1(1 \%)$ & $0(0 \%)$ & \\
\hline
\end{tabular}

Tabelle 3.4.2 zeigt die Ursache der Beatmung nach Einschätzung der behandelnden Ärzte der 126 aufgenommenen Patienten in zwei Gruppen: Fälle ohne und Fälle mit aufgetretener Infektion. Die Werte der Fälle ohne und mit Infektionen wurden mittels Fisher's exact test verglichen und das Ergebnis als $p$-Wert angegeben. Signifikante Werte sind nicht zu verzeichnen.

Die Verteilung der Beatmungsursache war in beiden Gruppen nicht signifikant unterschiedlich: nach Fisher's exact test waren alle p-Werte größer als fünf Prozent.

Die einzelnen operativen Eingriffe sind in Tabelle 3.1.3 aufgelistet.

\subsubsection{Therapie bei Entlassung}

Tabelle 3.4.3 listet die Therapieformen der Patienten mit versus ohne Infektion bei deren Entlassung auf. In beiden Gruppen wurde die Mehrzahl der Patienten, die entlassen werden konnten, mit einer ISB-Therapie über einen nicht-invasiven Zugang entlassen.

Zur Vollständigkeit werden die 14 Patienten mit aufgeführt, die während ihres Aufenthalts auf der Intensivstation verstarben. 


\begin{tabular}{|l|c|c|c|}
\hline & $\begin{array}{c}\text { Fälle ohne } \\
\text { Infektion } \\
(\mathbf{n = 1 0 3 )}\end{array}$ & $\begin{array}{c}\text { Fälle mit } \\
\text { Infektion } \\
(\mathbf{n}=\mathbf{2 3})\end{array}$ & $\mathbf{p}$-Wert \\
\hline Therapie bei Entlassung & $76(73,8 \%)$ & $11(47,8 \%)$ & 0,023 \\
\hline $\begin{array}{l}\text { ISB-Therapie über nicht-invasiven Zugang } \\
\text { (Maske) VCV bzw. PCV }\end{array}$ & $3(2,9 \%)$ & $2(8,7 \%)$ & 0,225 \\
\hline $\begin{array}{l}\text { ISB-Therapie über invasiven Zugang } \\
\text { (Tracheostoma) }\end{array}$ & $1(1 \%)$ & $0(0 \%)$ & 1,000 \\
\hline keine Beatmung mit $\mathrm{O}_{2}$-Gabe & $13(12,6 \%)$ & $6(26,1 \%)$ & 0,115 \\
\hline keine Beatmung ohne $\mathrm{O}_{2}$-Gabe & $10(9,7 \%)$ & $4(17,4 \%)$ & 0,284 \\
\hline \hline davon Verstorben & & & \\
\hline
\end{tabular}

Tabelle 3.4.3 zeigt die Therapie bei Entlassung der 126 aufgenommenen Patienten in den zwei Gruppen: Fälle ohne und Fälle mit aufgetretener Infektion. Die Werte der Fälle ohne und mit Infektionen wurden mittels Fisher's exact test verglichen und das Ergebnis als p-Wert angegeben. Das Signifikanzniveau wurde nach Bonferroni bei multiplen Testen korrigiert: $\alpha \leq 0,0125$. Signifikante Werte sind nicht zu verzeichnen.

Patienten mit aufgetretenen Infektionen wurden vermehrt invasiv beatmet entlassen. Jedoch trat kein signifikanter Unterschied nach Fisher's exact test auf.

\subsubsection{Mortalität}

Die Mortalität der Patienten, die während des stationären Aufenthalts und bis zu 90 Tage nach ihrer Entlassung verstarben, sind in Tabelle 3.4.4 aufgelistet.

\begin{tabular}{|l||c|c|c|}
\hline & $\begin{array}{c}\text { Fälle ohne Infektion } \\
(\mathbf{n = 1 0 3 )}\end{array}$ & $\begin{array}{c}\text { Fälle mit Infektion } \\
(\mathbf{n = 2 3})\end{array}$ & p-Wert \\
\hline davon stationär verstorben & $10(9,7 \%)$ & $4(17,4 \%)$ & 0,284 \\
\hline 90-Tage-Mortalität & $21(20,4 \%)$ & $7(30,4 \%)$ & 0,405 \\
\hline
\end{tabular}

Tabelle 3.4.4 zeigt die Mortalität (innerhalb 90 Tage nach Entlassung) der 126 aufgenommenen Patienten in den zwei Gruppen: Fälle ohne und Fälle mit aufgetretener Infektion. Die Werte der Fälle ohne und mit Infektionen wurden mittels Fisher's exact test verglichen und das Ergebnis als p-Wert angegeben. Signifikante Werte sind nicht zu verzeichnen.

Es verstarben mehr Patienten mit aufgetretener Infektion. Aber weder die 90-TageMortalität noch die Sterblichkeit während des stationären Aufenthalts waren nach Fisher's exact test signifikant unterschiedlich.

Unter den Patienten, die bis 90 Tage nach Entlassung verstarben, waren zwei an einer Pneumonie und vier an einem Atemwegsinfekt erkrankt. 


\subsubsection{Blutgase unter Spontanatmung}

Die Blutgase bei den Patienten ohne und mit Infektion werden zum einen bei deren Aufnahme und zum anderen bei deren Entlassung in der folgenden Tabelle 3.4.5 miteinander verglichen.

\begin{tabular}{|c|c|c|c|c|c|}
\hline & $\begin{array}{c}\text { Infektion } \\
\text { Diagnostiziert }\end{array}$ & $\mathbf{N}$ & Mittelwert & $\begin{array}{c}\text { Standard- } \\
\text { abweichung }\end{array}$ & $\begin{array}{c}\text { p-Wert im t-Test } \\
\text { bei unabhängigen } \\
\text { Stichproben }\end{array}$ \\
\hline \multicolumn{6}{|c|}{ bei Aufnahme } \\
\hline \multirow{2}{*}{ pH } & positiv & 19 & 7.42 & 0.07 & \multirow{2}{*}{0,812} \\
\hline & negativ & 98 & 7.41 & 0.08 & \\
\hline \multirow{2}{*}{$\mathrm{pCO}_{2}$ in $\mathrm{mmHg}$} & positiv & 19 & 52.07 & 18.16 & \multirow{2}{*}{0,227} \\
\hline & negativ & 98 & 52.95 & 13.32 & \\
\hline \multirow{2}{*}{$\mathrm{pO}_{2}$ in $\mathrm{mmHg}$} & positiv & 19 & 65.09 & 24.70 & \multirow{2}{*}{0,410} \\
\hline & negativ & 98 & 71.75 & 22.75 & \\
\hline \multirow{2}{*}{$\mathrm{HCO}_{3}$ - in $\mathrm{mmol} / \mathrm{l}$} & positiv & 19 & 31.121 & 7.30 & \multirow{2}{*}{0,924} \\
\hline & negativ & 98 & 30.71 & 5.77 & \\
\hline \multicolumn{6}{|c|}{ Bei Entlassung } \\
\hline \multirow{2}{*}{ pH } & positiv & 19 & 7.42 & 0.046 & \multirow{2}{*}{0,168} \\
\hline & negativ & 74 & 7.42 & 0.06 & \\
\hline \multirow{2}{*}{$\mathrm{pCO}_{2}$ in $\mathrm{mmHg}$} & positiv & 19 & 49.26 & 11.13 & \multirow{2}{*}{0,603} \\
\hline & negativ & 74 & 48.80 & 9.75 & \\
\hline \multirow{2}{*}{$\mathrm{pO}_{2}$ in $\mathrm{mmHg}$} & positiv & 19 & 69.58 & 17.31 & \multirow{2}{*}{0,127} \\
\hline & negativ & 74 & 65.31 & 14.63 & \\
\hline \multirow{2}{*}{$\mathrm{HCO}_{3}$ - in $\mathrm{mmol} / \mathrm{l}$} & positiv & 19 & 30.03 & 4.69 & \multirow{2}{*}{0,876} \\
\hline & negativ & 74 & 29.58 & 4.95 & \\
\hline
\end{tabular}

Tabelle 3.4.5 zeigt die Blutgase der Patienten mit und ohne Infektion bei deren Aufnahme und Entlassung. Die Werte sind als Mittelwert \pm Standardabweichung angegeben. „N“ gibt die Anzahl der Patienten, bei denen die Werte bekannt waren, an. Die Werte der Fälle mit und ohne Infektion wurden im t-Test für unverbundene Stichproben verglichen und das Ergebnis als $p$-Wert angegeben. Signifikante Werte sind nicht zu verzeichnen.

Vergleicht man die Blutgase der Patienten ohne und mit Infektion miteinander, so ergeben sich zwischen diesen keine statistisch signifikanten Unterschiede. 


\subsubsection{Lungenfunktion}

In Tabelle 3.4.6.1 werden die Lungenfunktionen der Patienten mit obstruktiver Grunderkrankung ohne und mit Infektionen gegenüber gestellt.

Tabelle 3.4.6.2 listet die Lungenfunktion bei den Patienten mit restriktiver Grunderkrankung auf, wobei hier keine Patienten mit Infektionen vorhanden sind.

\begin{tabular}{|c|c|c|c|c|c|}
\hline \multicolumn{6}{|c|}{$\begin{array}{l}\text { Fälle mit obstruktiver Grunderkrankung } \\
\qquad(\mathrm{n}=91)\end{array}$} \\
\hline \multirow[b]{2}{*}{ Alter in Jahren } & \multicolumn{2}{|c|}{$\begin{array}{l}\text { ohne Infektionen } \\
(\mathrm{n}=72)\end{array}$} & \multicolumn{2}{|c|}{$\begin{array}{l}\text { mit Infektionen } \\
(n=19)\end{array}$} & \multirow{2}{*}{$\frac{\text { p-Wert }}{0,251}$} \\
\hline & $68,58 \pm 9,552$ & $(n=72)$ & $66,63 \pm 11,84$ & $(n=19)$ & \\
\hline IVC (I) & $1,85 \pm 0,55$ & $(n=49)$ & $1,88 \pm, 52$ & $(n=14)$ & 0,983 \\
\hline IVC (\%) & $51,51 \pm 13,45$ & $(n=49)$ & $52,81 \pm 13,64$ & $(n=14)$ & 0,895 \\
\hline FEV1 (I) & $1,01 \pm 0,40$ & $(n=49)$ & $1,04 \pm 0,40$ & $(n=13)$ & 0,969 \\
\hline FEV1 (\%) & $37,59 \pm 14,18$ & $(n=49)$ & $38,48 \pm 14,76$ & $(n=13)$ & 0,955 \\
\hline FEV1/IVC (\%) & $55,20 \pm 17,29$ & $(n=49)$ & $53,98 \pm 14,24$ & $(n=13)$ & 0,757 \\
\hline $\mathrm{R}$ tot $\left(\mathrm{kPa}^{\star} \mathrm{s} / \mathrm{l}\right)$ & $0,90 \pm 0,78$ & $(n=48)$ & $0,78 \pm 0,39$ & $(n=13)$ & 0,354 \\
\hline RV/TLC (\%) & $68,93 \pm 14,29$ & $(n=48)$ & $68,18 \pm 11,06$ & $(n=13)$ & 0,801 \\
\hline
\end{tabular}

Tabelle 3.3.6.1 zeigt das Alter und die Lungenfunktion der 91 aufgenommenen Patienten mit obstruktiver Grunderkrankung in zwei Gruppen: Fälle ohne und Fälle mit aufgetretener Infektion. Die Werte sind als Mittelwert \pm Standardabweichung angegeben und in Klammern die Anzahl der Patienten, bei denen die Untersuchung durchgeführt wurde. Die Werte der Fälle ohne und mit Infektionen wurden im t-Test für unverbundene Stichproben verglichen und das Ergebnis als p-Wert angegeben. Signifikante Werte sind nicht zu verzeichnen.

\begin{tabular}{|c|c|c|}
\hline \multicolumn{3}{|c|}{$\begin{array}{l}\text { Fälle mit restriktiver Grunderkrankung ohne Infektion } \\
\qquad(\mathrm{n}=22)\end{array}$} \\
\hline Alter in Jahren & $37,14 \pm 19,46$ & $(n=22)$ \\
\hline IVC (I) & $1,18 \pm 0,87$ & $(n=15)$ \\
\hline IVC (\%) & $28,55 \pm 21,60$ & $(n=15)$ \\
\hline FEV1 (I) & $1,08 \pm 0,73$ & $(n=14)$ \\
\hline FEV1 (\%) & $31,76 \pm 22,42$ & $(n=14)$ \\
\hline FEV1/IVC (\%) & $90,05 \pm 7,97$ & $(n=15)$ \\
\hline $\mathrm{R}$ tot $\left(\mathrm{kPa}^{*} \mathrm{~s} / \mathrm{l}\right)$ & $0,37 \pm 0,69$ & $(n=11)$ \\
\hline RV/TLC (\%) & $57,93 \pm 27,58$ & $(n=12)$ \\
\hline
\end{tabular}

Tabelle 3.3.6.1 zeigt das Alter und die Lungenfunktion der 22 aufgenommenen Patienten mit restriktiver Grunderkrankung. Die Werte sind als Mittelwert \pm Standardabweichung angegeben und in Klammern die Anzahl der Patienten, bei denen die Untersuchung durchgeführt wurde. 
Vergleicht man die Lungenfunktion der Patienten ohne und der Patienten mit Infektion der Patientenklientel, die an einer obstruktiven Lungenerkrankung litt, so ergeben sich keine signifikanten Unterschiede.

Bei Patienten mit restriktiver Grunderkrankung konnte man, wie schon erwähnt, keine Infektion nachweisen.

\subsubsection{Atmungsfunktion}

Tabelle 3.4.7 stellt die Atmungsfunktion der Patienten mit versus ohne Infektion gegenüber.

\begin{tabular}{|l|c|c|c|c|c|}
\hline & \multicolumn{2}{|c|}{$\begin{array}{c}\text { Fälle ohne Infektion } \\
(\mathbf{n}=\mathbf{1 0 3})\end{array}$} & $p-W e r t$ & \multicolumn{2}{c|}{$\begin{array}{c}\text { Fälle mit Infektion } \\
(\mathbf{n}=\mathbf{2 3})\end{array}$} \\
\hline Alter in Jahren & $61,05 \pm 18,23$ & $(\mathrm{n}=103)$ & 0,055 & $66,22 \pm 12,24$ & $(\mathrm{n}=23)$ \\
\hline $\mathbf{P 0 , 1}$ & $0,38 \pm 0,24$ & $(\mathrm{n}=63)$ & 0,648 & $0,39 \pm 0,21$ & $(\mathrm{n}=11)$ \\
\hline $\mathbf{P 0 , 1 _ { \operatorname { m a x } }}$ & $1,62 \pm 1,13$ & $(\mathrm{n}=61)$ & 0,828 & $1,77 \pm 1,11$ & $(\mathrm{n}=11)$ \\
\hline $\mathbf{P I} \mathbf{m a x}_{\max }(\mathbf{k P a})$ & $3,62 \pm 2,44$ & $(\mathrm{n}=61)$ & 0,152 & $2,86 \pm 1,47$ & $(\mathrm{n}=11)$ \\
\hline $\mathbf{P 0 , 1 / P 0 , 1 _ { \operatorname { m a x } } ( \% )}$ & $30,54 \pm 21,93$ & $(\mathrm{n}=61)$ & 0,320 & $22,64 \pm 14,23$ & $(\mathrm{n}=11)$ \\
\hline $\mathbf{P 0 , 1 / P I}$ max $(\%)$ & $13,33 \pm 10,46$ & $(\mathrm{n}=61)$ & 0,324 & $12,91 \pm 6,61$ & $(\mathrm{n}=11)$ \\
\hline
\end{tabular}

Tabelle 3.4.7 zeigt das Alter und die Atmungsfunktion der aufgenommenen Patienten in den zwei Gruppen: Fälle ohne und Fälle mit aufgetretener Infektion. Die Werte sind als Mittelwert \pm Standardabweichung angegeben und in Klammern die Anzahl der Patienten, bei denen die Untersuchung durchgeführt wurde. Die Werte der Fälle ohne und mit Infektionen wurden im t-Test für unverbundene Stichproben verglichen und das Ergebnis als $p$-Wert angegeben. Signifikante Werte sind nicht zu verzeichnen.

Vergleicht man die Atmungsfunktion der Patienten ohne und mit Infektion miteinander, so ergeben sich zwischen diesen keine statistisch signifikanten Unterschiede. 


\section{Diskussion}

\subsection{Fälle mit Keimübertragungen}

Bei 14 Patienten, welches einem Anteil von 11\% der Patienten entspricht, die im Jahre 2010 auf der RICU des Klinikums Niederrhein stationär behandelt wurden, konnte eine Keimübertragung nachgewiesen werden. Bei 667 Erregernachweisen ist dies eine sehr geringe Menge, wenn man in Betracht zieht, welch enger Kontakt zwischen den Patienten bestand, da sie gemeinsam ein Zimmer belegten. Somit bestätigt sich die Annahme, dass es auf der RICU zu wenigen Keimübertragungen kam.

Als Keimübertragung wurde der Nachweis des gleichen Bakterienstammes mit gleichem Antibiogramm bei beiden Kontaktpersonen definiert. Zusätzlich musste der Bakterienstamm bei Patienten festgestellt werden, die sich zeitgleich stationär auf der RICU befanden. Einzuwenden wäre, dass keine DNA- oder Phagentypisierung vorgenommen wurde. Dies spielt jedoch nur eine untergeordnete Rolle, wenn bedacht wird, dass die Zahl der Übertragungen in diesem Fall vermindert und nicht erhöht worden wäre. Das Ergebnis stellt also die maximal mögliche Übertragungsrate dar.

Im Durchschnitt waren die Patienten mit bestätigter Keimübertragung 5,6 Jahre älter als die ohne Keimübertragung. Das Patientenalter spielt somit keine wesentliche Rolle.

Die Geschlechterverteilung war in beiden Gruppen sehr ähnlich. Der Anteil der männlichen Patienten lag zwischen $64,3 \%$ bei denen mit und $66,1 \%$ bei denen ohne diagnostizierte Keimübertragung.

\subsubsection{Grunderkrankungen}

In beiden Patientenkollektiven litt die Mehrzahl der Personen an einer obstruktiven Grunderkrankung. Bei den Patienten mit Keimübertragungen waren es $78,6 \%$ und bei denen ohne Keimübertragung $71,4 \%$. Im direkten Vergleich lagen bei den Fällen mit Keimübertragungen um 7,2 Prozentpunkte mehr Patienten mit obstruktiver Grunderkrankung als bei denen ohne Keimübertragung vor. 
Lediglich bei einem von 22 Patienten, die an einer restriktiven Grunderkrankung litten, fand eine Keimübertragung statt. Keimübertragungen fanden somit bei den Patienten mit restriktiver Grunderkrankung praktisch nicht und fast ausschließlich bei an COPD erkrankten Patienten statt.

Die beschriebenen Unterschiede sind statistisch nicht signifikant, da die Anzahl der Übertragungen gering ist. Dennoch weist es auf eine Tendenz hin. Eine Erklärung für diesen Sachverhalt, der eine vermehrte Keimübertragung bei obstruktiv Lungenerkrankten beschreibt, kann die meist schlechte Konstitution des Lungenparenchyms darstellen, wie dies bei Patienten mit COPD der Fall ist. Das Lungengewebe ist hier im Rahmen der Grunderkrankung vorgeschädigt, wodurch die Funktion der körpereigenen Barrieren gegen Erreger reduziert ist. Köhler erklärt dies dadurch, dass die vermehrte Anreicherung von intrapulmonalem mukösem Sekret einen guten Nährboden für Keime darstellt (2002).

\subsubsection{Ursache der Beatmung}

In der Gruppe der Patienten, bei denen die pulmonale Grunderkrankung zu einer Beatmung geführt hat, bestand kein signifikant erhöhtes Risiko, von einer Keimübertragung betroffen zu sein. Bei dem Kollektiv ohne und mit Keimübertragung ist der Anteil an Patienten, bei denen die Grunderkrankung zur Beatmung geführt hat, mit $64,3 \%$ und $68,3 \%$ vergleichbar.

Patienten, die aufgrund eines Infekts beatmet werden mussten, wiesen im Vergleich zu den anderen Patientengruppen eine erhöhte Anfälligkeit für eine Keimübertragung auf. Hier lagen rund 20\% mehr Patienten in der Patientengruppe mit Keimübertragungen vor $(28,6 \%)$ als bei denen ohne Keimübertragung (8,9\%). Dieser Zusammenhang ist nachvollziehbar, da eine Erregerübertragung die Grundlage einer Infektion darstellt, sowie eine Infektion zu einer Keimübertragung auf einen anderen Körper führen kann, da sie eine Vermehrung von pathogenen Keimen im Organismus darstellt.

Bei Patienten, die wegen akut eingetretener Ursachen, wie einem Pneumothorax, einer Reanimation, einer Intoxikation, einem Myokardinfarkt oder einer Lungenembolie, beatmet wurden, kamen Keimübertragungen seltener vor: Nur ein Patient und 
somit 7,1\% aller Patienten mit nachgewiesener Keimübertragung wurde wegen eines akuten Ereignisses, nämlich eines Pneumothorax, beatmet. In der Gruppe ohne Keimübertragungen waren es 16 Personen (14,4\%) der Patienten, die aufgrund einer akuten Ursache beatmet wurden. Bei einem akuten Anlass für eine Beatmung besteht in der Regel noch keine Vorschädigung der Lunge und das vorrangig gesunde Gewebe ist nach Köhler (2002) weniger anfällig für den Eintritt und die Vermehrung von Erregern.

\subsubsection{Liegedauer}

Der durchschnittliche stationäre Aufenthalt der Patienten auf der RICU betrug 11,44 Tage. Vergleicht man die Aufenthaltsdauer der Patienten mit nachgewiesener Keimübertragung mit der der Patienten, bei denen keine Übertragung diagnostiziert wurde, zeigt sich ein signifikanter Unterschied. Patienten mit übertragenem Keim lagen mit durchschnittlich 24,07 Tagen dementsprechend ca. zweieinhalbmal so lang stationär auf der RICU wie die Patienten ohne Keimübertragung (9,87 Tage).

Auch bei den Werten der minimalen und maximalen Aufenthaltsdauer sowie der Aufenthaltsdauer in Quartilen angegeben bestätigte sich das Bild, dass die Patienten mit Keimübertragungen eine längere Aufenthaltsdauer aufwiesen.

Somit war die absolute Dauer des Aufenthaltes ein signifikanter Risikofaktor für die Keimübertragung.

\subsubsection{Anzahl der Patientenkontakte und gemeinsame Liegedauer der Patien- ten}

Auch eine lange Kontaktdauer begünstigte eine Keimübertragung. Die Patientenkontakte, bei denen es zu einer Keimübertragung kam, bestanden durchschnittlich für 8,5 Tage, wohingegen die Kontaktdauern der Patienten, bei denen keine Keimübertragung nachzuweisen war, nur 6,2 Tage betrug. Dieser Unterschied war statistisch signifikant. 
Demgemäß steigt die Wahrscheinlichkeit einer Keimübertragung, je länger die Dauer der Patientenkontakte ist. Die Kontaktdauer ist somit auch ein signifikanter Risikofaktor für eine Keimübertragung.

Bemerkenswert ist, dass es nur bei 11 von 636 Patientenkontakten zu einer Keimübertragung kam. Dies entspricht nur 3\% aller Kontakte. Hiermit wird die Anwendung der Hygieneempfehlungen der DIGAB auf der RICU bestätigt (Laier-Groeneveld 1996). Die DIGAB plädierte darin für einen hygienischen und nicht sterilen Umgang bei beatmeten Patienten. Dies lässt sich durch die jetzigen Studienergebnisse unterstützen.

\subsubsection{Therapie bei Entlassung}

Die Patienten, bei denen Keimübertragungen stattgefunden hatten, waren schwerer pulmonal erkrankt. So wurden $21,4 \%$ von diesen mit einer ISB-Therapie über einen invasiven Zugang entlassen. Bei den Patienten ohne nachgewiesene Keimübertragungen war die Beatmung nur bei $2 \%$ der Patienten per Tracheostoma notwendig.

Die Entlassung ohne Beatmungstherapie war bei $17 \%$ der Patienten, bei denen keine Keimübertragung stattgefunden hatte, möglich. Im Gegensatz dazu konnte nur einer $(7,1 \%)$ der Patienten, bei denen eine Keimübertragung vorlag, ohne Beatmungstherapie entlassen werden. Dies erklärt sich aus der Schwere der Beanspruchung der Atmungsmuskulatur und aus den hyperkapnischen Blutgasen. Dieses Patientenkollektiv war anfälliger für Keime, da es kränker bzw. ihre chronische Erkrankung weiter fortgeschritten war. Vor allem war die Regeneration der Atmungsfunktion in der Patientengruppe mit nachgewiesenen Keimübertragungen schlechter (s.u.).

Eine Keimübertragung stellt somit einen Index für eine intensivere Beatmungs-therapie bei Entlassung dar.

Bemerkenswert ist, dass kein Patient mit einer ununterbrochenen Beatmung entlassen wurde, obwohl die Atmungsfunktion bei Aufnahme sehr schlecht war (s.u.). Nur 
4\% der Patienten mussten mit einer ISB-Therapie über ein Tracheostoma entlassen werden. Bei Veitshans (2002) waren dies in einer vergleichbaren Kohorte rund dreimal so viel $(11,5 \%)$.

\subsubsection{Mortalität und aufgetretene Infekte}

Die Mortalität der Patienten während der Hospitalisierung sowie bis 90 Tag nach inrer Entlassung von der Intensivstation war beim Patientenkollektiv mit nachgewiesener Keimübertragung mehr als doppelt so hoch wie die der Gruppe ohne Keimübertragung: $21 \%$ der Patienten mit Übertragungen starben noch auf der RICU und sogar $50 \%$ bis 90 Tage nach Entlassung. Ein signifikanter Unterschied lag bei der 90-Tage-Mortalität mit einem Signifikanzniveau von 0,05 vor. Dies zeigt den negativen Einfluss des Schweregrades der Erkrankung auf die Sterblichkeit. So lagen bei den Patienten mit erhöhter Mortalität bzw. Keimübertragungen vermehrt hyperkapnische Blutgase und niedrigere Sauerstoffpartialdrücke bei Entlassung vor (s. Tab. 3.2.7.2). Ob eine Keimübertragung direkten Einfluss auf die Mortalität hat, ist nicht eindeutig zu belegen. Sie gilt daher nicht als Mortalitätsfaktor, sondern erst die Folge davon, nämlich der Infekt: Die 90-Tage-Mortalität beim Patientenkollektiv mit Infektion war um rund 10 Prozentpunkte höher als bei dem ohne Infektion (s.u.).

Es trat bei $21 \%$ der Patienten, bei denen eine Keimübertragung diagnostiziert wurde, eine Infektion auf. In der Gruppe ohne Keimübertragungen hatte mit 18\% eine vergleichbar große Zahl an Patienten eine Infektion. Die 90-Tage-Mortalität der Patienten mit Infektionen und Keimübertragung war statistisch auch gleich. Dies zeigt, dass eine Keimübertragung nicht mit einem erhöhten Risiko verbunden ist, an einer Infektion zu erkranken und dadurch ein erhöhtes Mortalitätsrisiko zu haben.

Auch dieser Zusammenhang bestätigt die Sinnhaftigkeit der Anwendung der $\mathrm{Hy}$ gieneempfehlungen der DIGAB auf der RICU (Laier-Groeneveld 1996), weil kein Einfluss der Anzahl der Keimübertragungen auf die Mortalität nachgewiesen werden konnte und eine Verschärfung der Hygienemaßnahmen am ehesten die Keimverschleppung reduzieren würde. Zudem hätte dies keinen Einfluss auf die Infektionsrate, da diese nicht mit der Anzahl der Keimübertragungen korreliert. 


\subsubsection{Blutgase unter Spontanatmung}

\subsubsection{Blutgase bei Fällen mit versus ohne Keimübertragungen}

$\mathrm{Zu}$ allen Zeitpunkten lagen die durchschnittlichen $\mathrm{pCO}_{2-}, \mathrm{pO}_{2}-$ und $\mathrm{HCO}_{3}-$ Werte auBerhalb des Referenzbereiches, die pH-Werte lagen im Referenzbereich. Dies weist auf eine kompensierte respiratorische Azidose bei schon länger bestehender Atmungsinsuffizienz hin.

Die Blutgase der Patientengruppen mit Keimübertragung sind bei Aufnahme und bei Entlassung schlechter. Vor allem die $\mathrm{pCO}_{2}$-Werte bei Entlassung unterscheiden sich deutlich. Dies zeigt, dass die Atmungsfunktion bei den Patienten mit Keimübertragungen schlechter war und somit die Schwere der Erkrankung ein Risiko für eine Keimübertragung darstellt.

\subsubsection{Blutgase bei Aufnahme versus Entlassung}

In beiden Patientenkollektiven verbesserten sich die Blutgase während der $\mathrm{Be}$ handlung, was das Ziel der Beatmungstherapie war. Ein statistisch signifikanter Abfall liegt nur bei den Kohlenstoffdioxidpartialdrücken und den Bicarbonatwerten der Patienten ohne Keimübertragung vor. Es kam zu einer signifikanten Verbesserung des $\mathrm{pCO}_{2}$-Werts. In beiden Patientenkollektiven ist eine ventilatorische Regeneration zu verzeichnen. Die Patienten mit Keimübertragungen waren jedoch therapierefraktärer. Ein direkter negativer Einfluss einer Keimübertragung auf die Regeneration der Atmungsfunktion konnte nicht nachgewiesen werden.

\subsubsection{Lungenfunktion}

Die Inspiratorische Vitalkapazität in Prozent des Sollwerts und die FEV1/IVC weisen statistisch signifikante Unterschiede in den Patientengruppen ohne und mit nachgewiesenen Keimübertragungen bei einer obstruktiven Grunderkrankung auf: IVC weist in der Kategorie ohne Keimübertragung bessere Werte auf, FEV1/IVC weist in der Gruppe mit Keimübertragung bessere Werte auf. Die beiden Patienten- 
kollektive ähneln sich anhand ihrer Lungenfunktionen. Ein Einfluss der funktion auf die Wahrscheinlichkeit einer Keimübertragung bei obstruktiv Erkrankten kann nicht bestätigt werden.

Bei den Patienten mit restriktiven Grunderkrankungen liegen zu wenige Lungenfunktionsmessungen vor, als dass man die Ergebnisse miteinander vergleichen könnte. Es gab hier nur eine Keimübertragung.

\section{2 Übertragene Bakterienstämme}

Anhand der Erreger und Antibiogramme wurden Übertragungen von sieben unterschiedlichen Bakterienstämmen nachgewiesen. Kein Erreger wurde überdurchschnittlich häufiger als ein anderer übertragen. Die diagnostizierten Keime zählen vorwiegend zur Gruppe der häufigsten Erreger nosokomialer Infektionen, welche von Boucher et al. (2009) unter dem Sammelbegriff ESKAPE-Pathogene geführt werden. Darunter fielen zwei Staphylococcus aureus-Stämme (davon ein MRSA), ein Pseudomonas, ein Enterococcus faecium und ein Escherichia coli-Stamm. Multiresistente gramnegative Erreger wurden nicht übertragen.

\subsection{Fälle ohne und mit Infektionen}

Insgesamt wurden während des Untersuchungszeitraums 33 Infektionen diagnostiziert. Der größte Anteil mit 48\% waren die Infektionen des Respirationstraktes: Fünf Pneumonien (16\%) und zehn Atemwegsinfekte (31\%) wurden festgehalten. Vincent et al. (1995) beschrieben bei Patienten, die auf der Intensivstation versorgt wurden, einen Anteil von 46,9\% Pneumonien und 17,8\% Atemwegsinfektionen an allen aufgetretenen Infektionen. Daraus resultieren insgesamt $64,7 \%$ Infektionen des Respirationstraktes, welches $16,7 \%$ mehr als bei den Patienten auf der RICU waren. Dies ist bemerkenswert, da vorwiegend Lungenerkrankte auf der RICU behandelt werden und man ein erhöhtes Infektionsrisiko v.a. im Bereich des vorgeschädigten Respirationstraktes erwarten würde.

Bei 18\% der 126 Patienten trat eine Infektion auf. Patienten mit einer Infektion waren durchschnittlich 5 Jahre älter, und der Frauenanteil war bei den Fällen ohne 
Infektion größer. Die Unterschiede sind jedoch nicht signifikant. Ein erhöhtes Infektionsrisiko für ein bestimmtes Geschlecht oder Alter ist statistisch nicht nachzuweisen.

Die Liegedauer war bei den Patientengruppen mit und ohne aufgetretene Infektion annähernd gleich. Im Gegensatz dazu beschreibt Vincent (2003), dass eine stattgefundene Infektion auf einer modernen Intensivstation mit einer längeren Liegedauer verbunden war.

Dembinski und Rossaint (2008) zeigten, dass das Risiko für eine Beatmungspneumonie wesentlich mit der Beatmungsdauer korreliert: In den ersten 5 Beatmungstagen erhöht sich das Risiko annähernd um bis zu 3\% und mit jedem weiteren um 1\% pro Tag. Die durchschnittliche Beatmungszeit der 126 im Johanniter Krankenhaus Oberhausen untersuchten Patienten betrug 11,44 Tage. Wenn man nun das Risiko für eine Beatmungspneumonie auf diese Zeitspanne übertragen würde, läge es bei 21,44\%. Dies würde bei einem Patientenkollektiv von 126 zu einer Beatmungspneumonie bei rund 27 Patienten führen. Die Summe der im Jahr 2010 auf der RICU diagnostizierten Pneumonien betrug im Gegensatz dazu nur fünf. Es sind somit wesentlich weniger Pneumonien aufgetreten, als man es bei einer vergleichbaren Patientengruppe, die invasiv beatmet wird, erwartet hätte. Dies bestätigt, dass das Risiko, unter einer ISB-Therapie an einer Pneumonie zu erkranken, wesentlich geringer ist, als unter einer invasiven Beatmungstherapie.

\subsubsection{Grunderkrankungen}

Obwohl die Dauer des stationären Aufenthalts der Patienten mit obstruktiver und restriktiver Grunderkrankung annähernd gleich war (11,57 und 10,45 Tage s.Tab. 3.1.2), traten Infekte fast ausschließlich bei Patienten mit obstruktiver Grunderkrankung auf und bei keinem der Patienten mit restriktiver Grunderkrankung. Dies unterstützt die Annahme, dass die Grunderkrankung eine Rolle für die Infektionsanfälligkeit spielt: Für Patienten mit einer obstruktiven Lungenerkrankung liegt das Infektionsrisiko höher als bei denen mit einer restriktiven Grunderkrankung. 


\subsubsection{Ursache der Beatmung}

In der Gruppe der Patienten, bei denen die pulmonale Grunderkrankung zu einer Beatmung geführt hat, bestand ein gering erhöhtes Risiko, an einer Infektion zu erkranken: Bei dem Kollektiv mit Infektion war der Anteil an Patienten, bei denen die Grunderkrankung zur Beatmung geführt hat, um 8,8\% höher als bei dem Kollektiv, bei dem keine Infektion diagnostiziert wurde.

Wie auch im Vergleich der Patientengruppen ohne und mit nachgewiesener Keimübertragung traten Infektionen bei Patienten, die wegen akut eingetretener Ursachen, wie eines Pneumothorax, einer Reanimation, einer Intoxikation, eines Myokardinfarkts oder einer Lungenembolie, beatmet wurden, weniger häufig auf: Nur ein Patient und somit 7,1\% aller Patienten mit diagnostizierter Infektion wurde wegen eines akuten Ereignisses, nämlich einer Reanimation, beatmet. Bei einem akuten Anlass für eine Beatmung besteht in der Regel noch keine Vorschädigung der Lunge und das vorrangig gesunde Gewebe ist weniger anfällig für den Eintritt sowie die Vermehrung von Erregern und somit eine schlechtere Grundlage für eine Infektion.

\subsubsection{Therapie bei Entlassung}

Patienten mit einer stationär aufgetretenen Infektion waren durchschnittlich schwerer erkrankt und mussten bei Entlassung vermehrt über ein Tracheostoma bzw. einen invasiven Zugang beatmet werden.

Patienten, bei denen eine Infektion aufgetreten war, konnten öfter völlig ohne Beatmung entlassen werden. Die Ursache der Beatmung bei fünf dieser sechs Patienten, sprich ca. 83\%, war die Grunderkrankung (siehe Tabelle $6.4 \mathrm{im} \quad$ Anhang). Dies zeigt, dass trotz einer Infektion eine Beatmungstherapie erfolgreich ausgeleitet werden konnte und es zu einer Regeneration der respiratorischen Funktion kam.

Damit ist festzuhalten, dass ein Einfluss einer Infektion auf die Therapie bei Entlassung nicht signifikant nachgewiesen werden kann. 


\subsubsection{Mortalität}

Die 90-Tage-Mortalität beim Patientenkollektiv mit Infektion war um rund 10 Prozentpunkte höher als bei dem ohne Infektion. 17\% der Patienten mit und 10\% der Patienten ohne Infektion verstarben noch während der Behandlung auf der RICU.

Dies zeigt, je höher die Infektrate war, desto höher war auch die Sterblichkeit. Das ist entweder dadurch zu erklären, dass die Atmungsfunktion der Patienten mit Infektion schlechter war (s. Kapitel 4.3.7) oder dass eine Infektion direkten Einfluss auf die Mortalität hatte.

Es verstarben zwei der insgesamt fünf Patienten, die an Pneumonie erkrankten. Dies entspricht einer Letalität von 40\% bei Patienten mit Pneumonie. Im Jahre 2005 wurde eine Gesamtletalität bei Patienten mit Beatmungspneumonie von 30 bis $70 \%$ beschrieben (American Thoracic Society und Infectious Diseases Society of America 2005). Auf der RICU wurden primär Patienten behandelt, die schwer am Atmungstrakt erkrankt waren. Daher ist es bemerkenswert, dass gerade hier eine vergleichbar niedrige Letalität von $40 \%$ vorlag.

\subsubsection{Blutgase unter Spontanatmung}

$\mathrm{Zu}$ allen Zeitpunkten lagen die durchschnittlichen $\mathrm{pCO}_{2-}, \mathrm{pO}_{2}-$ und $\mathrm{HCO}_{3}-$ Werte auBerhalb des Referenzbereiches. Nur der $\mathrm{pO}_{2}-$ Wert bei Aufnahme der Patienten ohne Infektion lag mit $71,75 \mathrm{mmHg}$ noch knapp im Normbereich. Die gemittelten $\mathrm{pH}$-Werte lagen im Referenzbereich. Dies weist auf eine kompensierte respiratorische Azidose bei schon länger bestehender Atmungsinsuffizienz hin.

Auch unterscheiden sich die Blutgase der Patientengruppen ohne und mit Infektion bei Aufnahme nicht nennenswert und bei Entlassung nur minimal von einander. Dies zeigt, dass die Qualität der pulmonalen Funktion in beiden Patientenkollektiven vergleichbar ist, sprich sie sind ähnlich schwer erkrankt. Es kann nicht nachgewiesen werden, dass eine schlechte Blutgaskonstellation auch ein erhöhtes Risiko für eine Infektion darstellt. 


\subsubsection{Lungenfunktion}

Die Lungenfunktionen der Patientengruppen ohne und mit nachgewiesener Infektion bei einer obstruktiven Grunderkrankung weisen keine signifikanten Unterschiede auf. Die beiden Patientenkollektive ähneln sich bezüglich ihrer Lungenfunktion, und ein Einfluss der Lungenfunktion auf die Wahrscheinlichkeit einer Keimübertragung kann bei obstruktiv Erkrankten nicht bestätigt werden.

Bei den Patienten mit restriktiven Grunderkrankungen liegen zu wenige Lungenfunktionsmessungen vor, als dass man die Ergebnisse miteinander vergleichen könnte.

\subsubsection{Atmungsfunktion}

Im Vergleich der Atmungsfunktionsmessungen zeigen sich beim Patientenkollektiv mit Infektion schlechtere Werte. Somit ist bei schlechterer Atmungsfunktion von einem erhöhten Risiko, an einer Infektion zu erkranken, auszugehen.

Hervorzuheben ist, dass durchschnittlich alle behandelten Patienten eine sehr hohe Belastung der Atmungsmuskulatur hatten. Die Deutsche Atemwegsliga geht z.B. bei $\mathrm{Pl}_{\max }$ von einem unteren Grenzwert von 5,5 kPa bei Männern aus. Eine relevante Muskelschwäche könne man bei Werten größer 8,0 kPa ausschließen (Criée 2003). Jedoch hatten Patienten in unserer Studie weitaus niedrigere $\mathrm{PI}$ max-Werte, nämlich durchschnittlich $3,5 \mathrm{kPa}$. Normwerte des Mundverschlussdrucks während Ruheatmung liegen zwischen 0,1 und 0,15 kPa. Die Patienten auf der RICU weisen hingegen einen Durchschnittswert von $0,38 \mathrm{kPa}$ auf. Dies zeigt, dass die Patienten schwer erkrankt waren, welches die Notwendigkeit der Heimbeatmung belegt. 


\subsection{Schlussfolgerung}

Die vorliegende Untersuchung lässt erkennen, dass es während des Untersuchungszeitraums nur sehr wenige Übertragungen von Keimen auf der RICU gab. Nur bei 14 Patienten (11\%) und bei 11 von 363 Patientenkontakten (3\%) konnte eine Übertragung nachgewiesen werden.

Die Zahl von aufgetretenen Infekten auf der RICU im Vergleich zu konventionellen Intensivstationen war vergleichbar: Bei $18,25 \%$ der Patienten trat eine Infektion auf. Geffers et al. (2002) beschreiben eine Prävalenzrate von 15,3\% und die EPICStudie, welche die Prävalenz in 17 westeuropäischen Ländern untersuchte, eine von $21 \%$. Der Unterschied zwischen Infekten auf der RICU und auf konventionellen Intensivstationen liegt bei den Infekten des Respirationstraktes: Nur 49\% der Infektionen auf der RICU waren Pneumonien oder Atemwegsinfekte. Bei der Studie von Vincent et al. (1995) waren es 64,7\% auf Intensivstationen. Dies ist bemerkenswert, da vorwiegend Lungenerkrankte auf der RICU behandelt werden und man ein erhöhtes Infektionsrisiko v.a. im Bereich des vorgeschädigten und geschwächten Respirationstraktes erwarten würde.

Ein weiteres Ergebnis dieser Arbeit ist, dass das Risiko, an einem Infekt zu erkranken, nicht signifikant erhöht ist, wenn eine Keimübertragung stattgefunden hat. Rund $21 \%$ der Patienten mit versus 18\% der Patienten ohne Keimübertragungen erkrankten an einem Infekt. Dies legt nahe, dass ein Infekt nicht durch Verminderung der Übertragungsrate, also durch hygienische Maßnahmen, verhindert werden kann.

Dennoch zeigte es sich, dass eine erhöhte Mortalität bei den Patienten mit nachgewiesener Keimübertragung vorlag. Die 90-Tage-Mortalität ergab hier 50\%. Im Gegensatz dazu betrug bei der Referenzgruppe die Mortalität nur ca. 19\%. Auch war die Mortalität bei Patienten mit Infektionen höher: $30,4 \%$ bei den Fällen mit versus $20,4 \%$ bei Fällen ohne diagnostizierte Infektion. Somit erleiden Patienten mit schwerer Erkrankung häufiger einen Infekt, der dann zu einer erhöhten Sterberate auch nach Ausheilung im Verlauf führt. 
Die Daten bezüglich der Beatmungstherapie bei Entlassung beschreiben einen ähnlichen Trend. Patienten ohne nachgewiesene Keimübertragung wurden im Durchschnitt weniger intensiv beatmet entlassen. 17\% von diesen konnten ganz ohne Beatmungstherapie und nur 2\% mussten mit ISB-Therapie über einen invasiven Zugang entlassen werden. Im Vergleich hierzu wurden nur 7,1\% der Patienten mit belegter Keimübertragung ohne Beatmungstherapie entlassen und $21 \%$ mussten mit ISB-Therapie über einen invasiven Zugang entlassen werden.

Bezüglich der Schwere der pulmonalen Funktionseinschränkung stellte sich eine andere Tendenz heraus. Es lag im Bezug auf diese keine erhöhte oder niedrigere Prävalenz weder zur Keimbesiedlung durch eine Übertragung noch zur Erkrankung an einem Infekt vor. Die Lungenfunktion und auch die Blutgase im Vergleich der Patienten ohne und mit nachgewiesener Keimübertragung oder identifiziertem Infekt waren nicht signifikant unterschiedlich. Auch die Blutgase, die bei der Aufnahme auf und der Entlassung von der RICU erhoben wurden, zeigten in den verschiedenen Kollektiven vergleichbare Veränderungen. Bei Patienten ohne und mit nachgewiesener Keimübertragung und bei Patienten ohne und mit diagnostizierter Infektion kam es von der stationären Aufnahme bis zur Entlassung zu einer ähnlichen pulmonalen Regeneration.

Es zeigte sich, dass eher die Grunderkrankung Einfluss auf eine Keimübertragung und Infekte hatte und nicht in erster Linie die Schwere der aktuellen pulmonalen Funktionseinschränkung. So traten Infekte nur bei obstruktiv Lungenerkrankten auf, nämlich bei insgesamt 23 Patienten (18,25\%). Hier ist das Lungenparenchym vorgeschädigt, in den Luftwegen sammelt sich vermehrt Bronchialsekret bzw. Mucus, und es besteht daher eine erhöhte Disposition einer Keimbesiedlung. Bei Patienten mit restriktiver Grunderkrankung trat keine Infektion auf. Primär ist bei diesem Patientenkollektiv nur die Funktion der Atmungspumpe beeinträchtigt, das Lungengewebe ist intakt und stellt kein erhöhtes Risiko für den Keimeintritt und folglich deren Vermehrung dar.

In Bezug auf die Ursache, weswegen eine Beatmung notwendig war, wird diese Tendenz untermauert. Patienten mit eingeleiteter Beatmung aufgrund von akut eingetretenen Ursachen, wie z.B. einem Pneumothorax, einer Reanimation oder Intoxikation, hatten weniger Keimübertragungen und Infektionen, da in der Regel hier 
noch keine Vorschädigung der Lunge besteht und das vorrangig gesunde Gewebe weniger anfällig für den Eintritt oder die Vermehrung von Erregern ist.

Bei lediglich 5,9\% (einer von 17) der Patienten mit Beatmung wegen einer akuten Ursache wurde eine Keimübertragung sowie eine Infektion nachgewiesen.

Wir konnten zeigen, dass die Liegedauer mit der Anzahl von Keimübertragungen positiv korreliert. Patienten mit nachgewiesener Keimtransferierung lagen 24,07 statt 9,87 Tage stationär auf der RICU. Da, wie oben beschrieben, eine Keimübertragung nicht vermehrt zu Infekten führt, kann man davon ausgehen, dass eine lange Liegedauer eher eine Keimübertragung begünstigt, als dass eine Keimübertragung sich negativ auf die Liegedauer auswirken würde. Dies unterstreicht auch der nachgewiesene Sachverhalt, dass eine lange Kontaktdauer eine Keimübertragung begünstigt. Die durchschnittliche Kontaktdauer der Patientenpaare, bei denen eine Übertragung stattfand, betrug 8,5 Tage. Dies sind 2,3 Tage bzw. 27\%Punkte mehr als bei Patientenpaaren ohne nachgewiesene Keimübertragung.

Diese Arbeit konnte weder eine gehäufte Keimübertragung noch deren eindeutigen Einfluss auf den Outcome der behandelten Patienten zeigen. Daher wäre auch eine Änderung der damit zusammenhängenden Hygienemaßnahmen auf der RICU bzw. Stationen, auf denen Patienten mit vergleichbaren Erkrankungen, Gesundheitszuständen und mit ähnlichen Therapieansätzen behandelt werden, nicht zu postulieren. 


\section{Zusammenfassung}

Übertragungen von Keimen von einem Patienten zum anderen und nosokomiale Infekte spielen eine große Rolle auf der Intensivstation. Intubation und Sedation des Patienten tragen ebenso wie invasive Zugänge zu diesem Risiko bei.

Studien haben gezeigt, dass es bei chronischer Atmungsschwäche nach Einleitung einer nicht-invasiven Beatmung zu einem Rückgang der Infekte kommen kann. Gelingt es durch eine frühzeitige nicht-invasive Beatmung, eine Intubation zu vermeiden, so steigt die absolute Überlebensrate um etwa 20\%. Die Wahrscheinlichkeit einer nosokomialen Pneumonie reduziert sich erheblich. Werden invasiv beatmete Patienten an eine Maskenbeatmung transferiert, so steigt die Überlebensrate ebenfalls um absolute $20 \%$. Nosokomiale Pneumonien traten in der Untersuchung von Nava et al. (1998) im nicht-invasiv beatmeten Patienten-kollektiv keine auf, in der invasiv beatmeten Gruppe dagegen in $20 \%$.

Die Arbeitsgemeinschaft Heimbeatmung und Respiratorentwöhnung e.V., heute DIGAB, definierte daher abweichende Hygienekriterien für so behandelte Patienten (Laier-Groeneveld 1996).

In der respiratorischen Beatmungsintensivstation des Klinikums Niederrhein werden bei Aufnahme, bei Entlassung, wöchentlich und im Rahmen von Bronchoskopien viele mikrobiologische Untersuchungen bei jedem Patienten standardmäßig durchgeführt.

Die vorliegenden Studie untersuchte alle im Jahr 2010 erhobenen mikrobiologischen Daten auf Keimübertragungen von einem Patienten zum anderen und auf die Bedeutung der Übertragungen auf Infektionsrisiko, Grunderkrankung, Therapie und Prognose sowohl während des stationären Aufenthaltes als auch in der Zeit nach Entlassung aus der Klinik. Die Bedeutung der Keimübertragungen wurde untersucht in Hinblick auf die Grunderkrankung und den Schweregrad der Erkrankung, definiert durch Lungenfunktion, Atmungsfunktion, Blutgase und Beatmungsabhängigkeit. Als Keimübertragung wurde der Nachweis des gleichen Bakterienstammes mit gleichem Antibiogramm bei zwei Patienten definiert, die sich zeitgleich auf der RICU befanden.

Zudem wurden alle nosokomialen Infektionen während desselben Zeitraums erfasst und auch ihre Bedeutung in Bezug auf Grunderkrankung, Therapie und Prognose ausgewertet. 
Die Ergebnisse zeigen, dass es sehr wenige Keimübertragungen auf der RICU gab. Nur bei $11 \%$ der Patienten und bei $3 \%$ von 363 Patientenkontakten trat eine Übertragung auf. Je länger die Patienten auf der RICU behandelt wurden bzw. je länger ihre Kontakt- und Liegedauern waren, desto höher war das Risiko für eine Keimübertragung. Die Zahl der aufgetretenen Infekte war vergleichbar mit denen auf konventionellen Intensivstationen, wobei weniger Infektionen im Bereich des Respirationstraktes zu vermerken waren. Dies ist bemerkenswert, da primär Lungenerkrankte auf der RICU therapiert wurden und bei dieser Patientenklientel eine erhöhte Infektionsrate v.a. im Rahmen des vorgeschädigten und geschwächten Respirationstraktes zu erwarten wäre. Ein erhöhtes Risiko, an einem Infekt zu erkranken, wenn Keimübertragungen statt gefunden haben, konnte nicht nachgewiesen werden. Es lag jedoch eine erhöhte Mortalität bei Patienten mit nachgewiesener Keimübertragung oder Infektion vor. Dies ist dadurch zu erklären, dass die Patienten mit Keimübertragen zum einen schwerer erkrankt waren: Die Atmungsfunktion war schlechter, was die Blutgase bei Aufnahme und Entlassung belegen, und die Beatmungstherapie bei Entlassung war intensiver. Zum anderen waren es vorwiegend obstruktiv Lungenerkrankte, bei denen der Grund für die Einleitung der Beatmungstherapie eine chronische Erkrankung war. Hier ist das Lungenparenchym im Rahmen der Grunderkrankung vorgeschädigt und die Funktion der körpereigenen Barriere gegen Erreger ist reduziert.

Die Studienergebnisse lassen auf keine gehäufte Keimübertragung bei den auf der RICU behandelten Patienten schließen. Auch ein Einfluss dadurch auf den Outcome dieses Patientenkollektivs ist nicht nachzuweisen. Daraus kann gefolgert werden, dass eine Änderung der Hygienemaßnahmen, wie sie die DIGAB für die RICU bzw. für die Patientenbehandlung mit vergleichbarem Setting empfiehlt, nicht zu postulieren wäre. 


\section{Anhang}

\subsection{Tabelle 6.1}

\begin{tabular}{|c|c|c|}
\hline & $\begin{array}{c}\text { Fälle mit } \\
\text { Keimübertragungen } \\
(n=14)\end{array}$ & $\begin{array}{c}\text { Fälle ohne } \\
\text { Keimübertragungen } \\
(n=112)\end{array}$ \\
\hline Keine definierte Grunderkrankung & $1(7,1 \%)$ & $0(0 \%)$ \\
\hline Obstruktive Grunderkrankung & $11(78,6 \%)$ & $80(71,4 \%)$ \\
\hline davon COPD & $11(78,6 \%)$ & $79(70,5 \%)$ \\
\hline davon Asthma bronchiale & $0(0 \%)$ & $1(0,9 \%)$ \\
\hline Restriktive Grunderkrankung & $1(7,1 \%)$ & $21(18,8 \%)$ \\
\hline davon ALS & $0(0 \%)$ & $4(3,6 \%)$ \\
\hline davon Muskeldystrophie Duchenne & $1(7,1 \%)$ & $11(9,8 \%)$ \\
\hline davon Gliedergürtelmuskeldystrophie & $0(0 \%)$ & $1(0,9 \%)$ \\
\hline davon Spinale Muskelatrophie & $0(0 \%)$ & $1(0,9 \%)$ \\
\hline davon Multiple Sklerose & $0(0 \%)$ & $1(0,9 \%)$ \\
\hline davon Morbus Parkinson & $0(0 \%)$ & $1(0,9 \%)$ \\
\hline davon Mukopolysaccharidose Typ IV & $0(0 \%)$ & $1(0,9 \%)$ \\
\hline $\begin{array}{l}\text { davon Tetraparese nach Basilarisa- } \\
\text { neurysma }\end{array}$ & $0(0 \%)$ & $1(0,9 \%)$ \\
\hline Andere & $1(7,1 \%)$ & $11(9,8 \%)$ \\
\hline Lungenfibrose & $0(0 \%)$ & $3(2,7 \%)$ \\
\hline Bronchialkarzinom & $0(0 \%)$ & $2(1,8 \%)$ \\
\hline Herzinsuffizienz & $0(0 \%)$ & $2(1,8 \%)$ \\
\hline zentrale Atemregulationsstörung & $1(7,1 \%)$ & $1(0,9 \%)$ \\
\hline $\begin{array}{l}\text { Z.n. Lungenteilresektion bei Bronchial- } \\
\text { karzinom }\end{array}$ & $0(0 \%)$ & $1(0,9 \%)$ \\
\hline $\begin{array}{l}\text { OSAS: Obesitas-Hypoventilationssyn- } \\
\text { drom }\end{array}$ & $0(0 \%)$ & $1(0,9 \%)$ \\
\hline Z.n. Mammakarzinom & $0(0 \%)$ & $1(0,9 \%)$ \\
\hline
\end{tabular}

Tab. 6.1 Grunderkrankungen der 126 aufgenommenen Patienten in den zwei Untergruppen: Fälle mit und Fälle ohne nachgewiesener Keimübertragung 


\subsection{Tabelle 6.2}

\begin{tabular}{|c||c||c|c|c|}
\hline \multirow{2}{*}{ Patientenpaar } & \multirow{2}{*}{\multicolumn{1}{|c||}{ übertragener Keim }} & \multicolumn{3}{c|}{ Liegedauer in Tagen auf: } \\
\cline { 3 - 5 } & & RICU & Intensivstation & Normalstation \\
\hline A & MRSA & 4 & - & - \\
\hline B & Staph. Aureus & 2 & 4 & - \\
\hline C & Pseudomonas & 18 & 4 & - \\
\hline D & Hämophilus influenzae & 26 & - & - \\
\hline E & \multirow{3}{*}{ Enterococcus ssp. } & 13 & - & - \\
\cline { 3 - 5 } & & 7 & 31 & 16 \\
\hline F & \multirow{2}{*}{ Enterococcus faecium } & 11 & - & 8 \\
\hline H & \multirow{2}{*}{ E. Coli } & 4 & - & - \\
\hline I & & 9 & - & - \\
\hline J & & 7 & - & - \\
\hline
\end{tabular}

Tab. 6.2 zeigt den Keim (Bakterienstamm mit identischem Antibiogramm), der von einem zum anderen Patienten eines Patientenpaares übertragen wurde. Die gemeinsame stationäre Aufenthaltsdauer auf der RICU, Intensiv- und Normalstation der Patienten eines Patientenpaares ist in Tagen angegeben. 


\subsection{Tabelle 6.3}

\begin{tabular}{|c|c|c|}
\hline & $\begin{array}{l}\text { Fälle ohne Infektion } \\
\qquad(n=103)\end{array}$ & $\begin{array}{l}\text { Fälle mit Infektion } \\
\qquad(\mathrm{n}=23)\end{array}$ \\
\hline Keine definierte Grunderkrankung & $1(1 \%)$ & $0(0 \%)$ \\
\hline Obstruktive Grunderkrankung & $72(69,9 \%)$ & $19(82,6 \%)$ \\
\hline davon COPD & $71(68,9 \%)$ & $19(82,6 \%)$ \\
\hline davon Asthma bronchiale & $1(1 \%)$ & $0(0 \%)$ \\
\hline Restriktive Grunderkrankung & $22(21,4 \%)$ & $0(0 \%)$ \\
\hline davon ALS & $4(3,9 \%)$ & $0(0 \%)$ \\
\hline davon Muskeldystrophie Duchenne & $12(11,7 \%)$ & $0(0 \%)$ \\
\hline davon Gliedergürtelmuskeldystrophie & $1(1 \%)$ & $0(0 \%)$ \\
\hline davon Spinale Muskelatrophie & $1(1 \%)$ & $0(0 \%)$ \\
\hline davon Multiple Sklerose & $1(1 \%)$ & $0(0 \%)$ \\
\hline davon Morbus Parkinson & $1(1 \%)$ & $0(0 \%)$ \\
\hline davon Mukopolysaccharidose Typ IV & $1(1 \%)$ & $0(0 \%)$ \\
\hline davon Tetraparese nach Basilarisaneurysma & $1(1 \%)$ & $0(0 \%)$ \\
\hline Andere Grunderkrankung & $8(7,8 \%)$ & $4(17,4 \%)$ \\
\hline davon Lungenfibrose & $3(2,9 \%)$ & $0(0 \%)$ \\
\hline davon Bronchialkarzinom & $1(1 \%)$ & $1(4,3 \%)$ \\
\hline davon Herzinsuffizienz & $1(1 \%)$ & $1(4,3 \%)$ \\
\hline davon zentrale Atemregulationsstörung & $1(1 \%)$ & $1(4,3 \%)$ \\
\hline $\begin{array}{l}\text { davon Z.n. Lungenteilresektion bei Bronchial- } \\
\text { karzinom }\end{array}$ & $0(0 \%)$ & $1(4,3 \%)$ \\
\hline $\begin{array}{l}\text { davon OSAS: Obesitas-Hypoventilationssyn- } \\
\text { drom }\end{array}$ & $1(1 \%)$ & $1(4,3 \%)$ \\
\hline davon Z.n. Mammakarzinom & $1(1 \%)$ & $1(4,3 \%)$ \\
\hline
\end{tabular}

Tab. 6.3 Grunderkrankungen der 126 aufgenommenen Patienten in zwei Gruppen: Fälle ohne und Fälle mit aufgetretener Infektion 


\subsection{Tabelle 6.4}

Patienten mit Infektion: Ursache der Beatmung und Therapie bei Entlassung im Vergleich

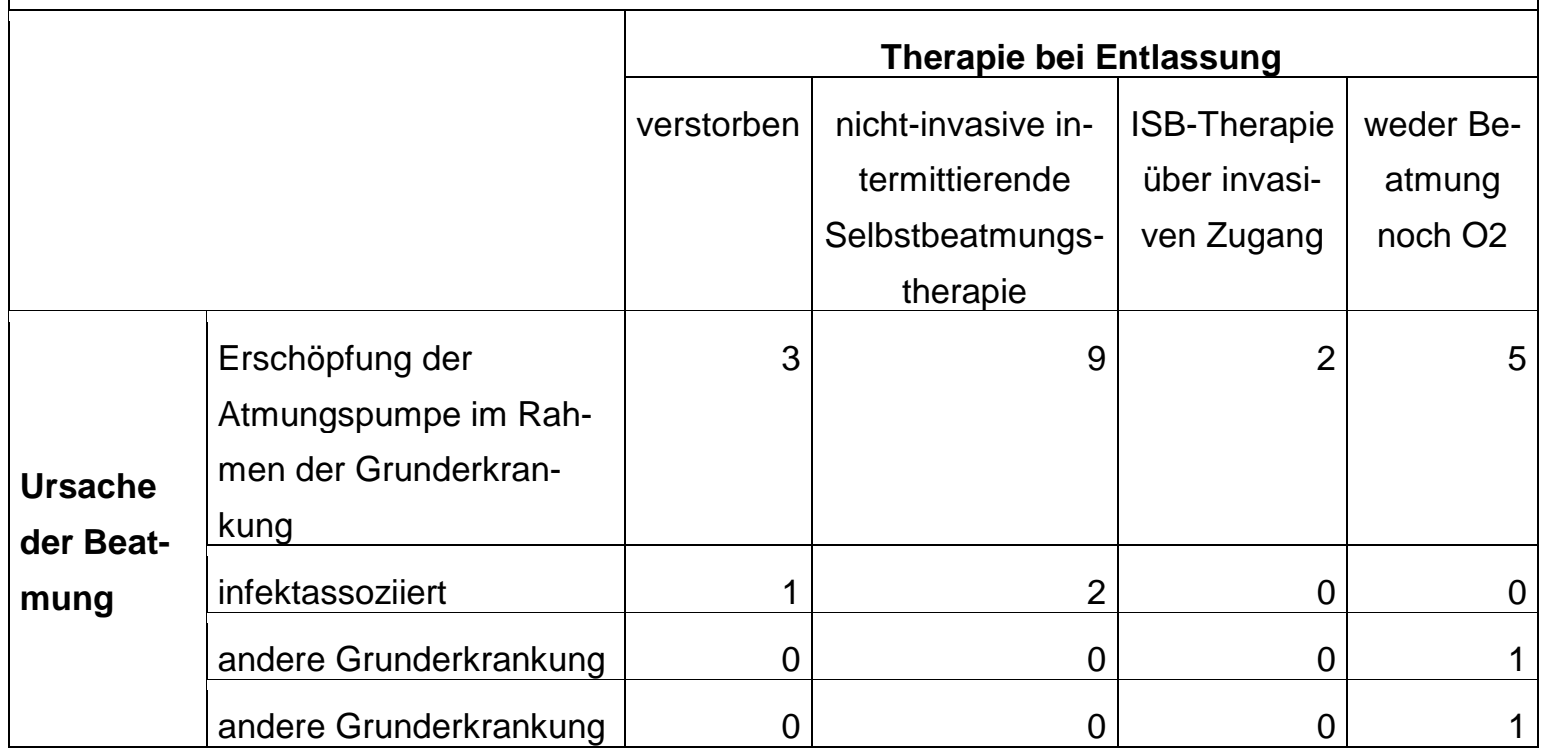

Tabelle 6.4 zeigt eine Kreuztabelle der Patienten mit diagnostizierter Infektion. Die Charakteristika der Patienten, die verglichen wurden, sind: „Ursache der Beatmung“ und „Therapie bei Entlassung". 


\section{Literaturverzeichnis}

American Thoracic Society und Infectious Diseases Society of America (2005): Guidelines for the management of adults with hospital-acquired, ventilatorassociated and healthcare-associated pneumonia. Am J Respir Crit Care Med $\underline{171}, 388-416$

Bertolini G, Confalonieri M, Rossi C, Rossi G, Simini B, Gorini M, Corrado A, GiViTI (Gruppo italiano per la Valutazione degli interventi in Terapia Intensiva) Group und Aipo (Associazione Italiana Pneumologi Ospedalieri) Group (2005): Costs of the COPD. Differences between intensive care unit and respiratory intermediate care unit. Respir Med 99, 894-900

Bösch D, Criée CP: Lungenfunktionsprüfung. 3. Auflage; Springer Verlag, Berlin/ Heidelberg 2013

Boucher HW, Talbot GH, Bradley JS, Edwards JE, Gilbert D, Rice LB, Scheld M, Spellberg B, Bartlett J (2009): Bad Bugs, No Drugs: No ESKAPE! An Update from the Infectious Diseases Society of America. Clin Infect Dis $\underline{48}, 1-$ 12

Brochard L, Mancebo J, Wysocki M, Lofaso F, Conti G, Rauss A, Simonneau G, Benito S, Gasparetto A, Lemaire F (1995): Noninvasive Ventilation for Acute Exacerbations of Chronic Obstructive Pulmonary Disease. N Engl J Med $\underline{333}$, 817-822

Bunburaphong T, Imanaka H, Nishimura M, Hess D, Kacmarek RM (1997): Performance characteristics of bilevel pressure ventilators: a lung model study. Chest 111, 1050-1060

Burchardi H, Schönhofer B (2000): Invasive oder nicht-invasive Beatmung? Kein entweder - oder! Pneumologie $\underline{54}, 2-4$

Burchardi H, Kuhlen R, Schönhofer B, Müller E, Criée CP, Welte T (2002): Konsensus-Statement zu Indikationen, Möglichkeiten und Durchführung der nichtinvasiven Beatmung bei der akuten respiratorischen Insuffizienz. Anaesthesist $\underline{51}, 33-41$

Carlucci A, Richard JC, Wysocki M, Lepage E, Brochard L (2001): Noninvasive versus conventional mechanical ventilation. An epidemiologic survey. Am J Respir Crit Care Med $\underline{163}, 874-880$ 
Corrado A, Roussos C, Ambrosino N, Confalonieri M, Cuvelier A, Elliott M, Ferrer

M, Gorini M, Gurkan O, Muir JF (2002): Respiratory intermediate care units:

a European survey. Eur Respir J 므, 1343-1350

Criée CP (2003): Empfehlungen der Deutschen Atemwegsliga zur Messung der inspiratorischen Muskelfunktion. Pneumologie $\underline{57}, 98-100$

Dembinski R, Rossaint R (2008): Ventilatorassoziierte Pneumonie. Anaesthesist $\underline{30}$, 122-129

Dettenkofer M, Meyer E: Hygiene in der Intensivmedizin. In: Burchardi H, Larsen R, Marx G, Muhl E, Schölmerich J (Hrsg.): Die Intensivmedizin. 11. Auflage; Springer Verlag, Berlin u.a. 2011, 37-46

Dietrich ES, Demmler M, Schulgen G, Fekec K, Mast O, Pelz K, Daschner FD (2002): Nosocomial pneumonia: a cost-of-illness analysis. Infection $\underline{30}, 61$ 67

Drinker PA, McKhann CF (1986): Landmark perspective: The iron lung. First Practical Means of Respiratory Support. JAMA 255(11), 1476-1480

Elpern E, Silver M, Rosen R, Bone R (1991): The non-invasive respiratory care unit. Pattern of use and financial implications. Chest $\underline{99}, 205$

Ewig S: Pneumonien. In: Burchardi H, Larsen R, Marx G, Muhl E, Schölmerich J (Hrsg.): Die Intensivmedizin. 11 Auflage; Springer Verlag, Berlin u.a. 2011, 487-505

Gastmeier P, Geffers C (2008): Nosokomiale Infektionen in Deutschland: Wie viele gibt es wirklich? Dtsch Med Wochenschr 133, 1111-1115

Gastmeier P, Kampf G, Wischnewski N, Hauer T, Schulgen Gl, Schumacher M, Daschner F, Rüden H (1998): Prevalence of nosocomial infections in representative German hospitals. J Hosp Infect $\underline{38}$, 37-49

Gastmeier P, Weist K, Weight O, Rüden H (1999): Prävention nosokomialer Infektionen in der Intensivmedizin und im OP. Anaesthesist $\underline{48}, 575-590$

Geffers C, Gastmeier P, Rüden H: Nosokomiale Infektionen. In: Robert Koch-Institut (Hrsg.): Gesundheitsberichterstattung des Bundes, Heft 8. Robert Koch-Institut, Berlin 2002, 1-18

Glenister HM, Emori WR, Horan TC, Hughes JM (1998): CDC definitions for nosocomial infections. Am J Infect Control 16, 128-140 
Gorgs O (2013): Weiterbildung Intermediate Care. [Online im Internet:] URL:

http://www.rbk.de/bildung/weiterbildung/intermediate-care.html, http://www.rbk.de/fileadmin/downloads/infomaterial/ 7_kurs_und_vortragsangebote/Brosch\%C3\%BCre_Fort-_und_Weiterbildung_2014_Teil_1.pdf [Stand: 13.05.2014, 07:55].

Grundmann H, Barwolff S, Tami A, Behnke M, Schwab F, Geffers C, Halle E, Gobel UB, Schiller R, Jonas D (2005): How many infections are caused by patientto-patient transmission in intensive care units? Crit Care Med 33, 946-951

Hintzenstern U, Bein T, Köhnlein T, Welte T, Erbguth F: Aspekte rund um die Beatmung. In: Hintzenstern U und Bein T (Hrsg.): Praxisbuch Beatmung. 5. Auflage; Elsevier, Urban \& Fischer, München 2012, 159-198

Ickler H (2011): Innerbetriebliche Fort- und Weiterbildung, Weiterbildung Intensivüberwachungspflege (IMC). [Online im Internet:] URL: http://www.uniklinik-ulm.de/fileadmin/Zentrale_Einrichtungen/Pflegedienst/ ibfw/ Info_IMC_2011_Intranet.pdf [Stand: 07.05.2014, 17:29].

International Consensus Conferences in Intensive Care Medicine (2001): Noninvasive positive pressure ventilation in acute Respiratory failure. Am J Respir Crit Care Med 163, 283-291

Jantzen JPAH: Intensivtherapie nach neurochirurgischen Eingriffen - intrakranielle Blutung, Schädel-Hirn-Trauma, Rückenmarkverletzung. In: Burchardi H, Larsen R, Kuhlen R, Jauch KW, Schölmerich J (Hrsg.): Die Intensivmedizin. 10. Auflage; Springer Medizin Verlag, Heidelberg 2008, 1005-1027

Kappstein I: Nosokomiale Infektionen, Prävention-Labordiagnostik-Antimikrobielle Therapie. 4. Auflage; Georg Thieme Verlag, Stuttgart/New York 2009 Kerwat K, Graf J, Wulf H (2010): Krankenhaushygiene - Nosokomiale Infektionen. Anasthesiol Intensivmed Notfallmed Schmerzther $\underline{45}$ (1), 30-31 Köhler D: Physiologie der Atmung. In: Becker HF, Schönhofer B, Burchardi H (Hrsg.): Nicht-invasive Beatmung. Blackwell Wissenschaften, Berlin/Wien 2002, 5-38 
Köhnlein T, Windisch W, Köhler D, Drabik A, Geiseler J, Hartl S, Karg O, LaierGroeneveld G, Nava S, Schönhofer B (2014): Non-invasive positive pressure ventilation for the treatment of severe stable chronic obstructive pulmonary disease: a prospective, multicentre, randomised, controlled clinical trial. Lancet Respir Med 2, 698-705

Kramer A, Christiansen B, Exner M, Rotter M (2000): Händehygiene, Mitteilung der Kommission für Krankenhaushygiene und Infektionsprävention am Robert Koch-Institut. Bundesgesundheitsbl - Gesundheitsforsch - Gesundheitsschutz $\underline{43}, 230-233$

Kuhlen R, Dembinski R: Maschinelle Beatmung und Entwöhnung von der Beatmung. In: Burchardi H, Larsen R, Kuhlen R, Jauch KW, Schölmerich J (Hrsg.): Die Intensivmedizin. 10. Auflage; Springer Medizin Verlag, Heidelberg 2008, 513-530

Laier-Groeneveld G (1996): Guidelines for management of home ventilation patients. Home and Long-Term Ventilation Working Group. Med Klin 91, 615616

Laier-Groeneveld G: Die intermittierende Selbstbeatmung. Med. Diss. Göttingen 1998

Laier-Groeneveld G, Criée CP (1994): Standortbestimmung und Perspektiven der intermittierenden Selbstbeatmung (ISB): Methoden, Ergebnisse und Perspektiven. Med Klin $\underline{89}$, 7-10

Laier-Groenevled G, Criée CP, Bänsch S, Hüttemann U (1989a): Die nichtinvasive intermittierende Selbstbeatmung. Atemwegs-Lungenkr $\underline{8}, 339-440$

Laier-Groeneveld G, Hüttemann U, Criée CP: Die intermittierende nicht-invasive Selbstbeatmung - Beatmung auf der Normalstation als effektive Therapie der chronischen ventilatorischen Insuffizienz. In: Grosser KD, Just H, Schuster HP: (Hrsg.): Intensivmedizin und Notfallmedizin. Band 26; Dr. Dietrich Steinkopff Verlag, Darmstadt 1989b, 392-397

Laier-Groeneveld G, Criée CP, Hüttemann U (1990): Self-ventilation at home in chronic respiratory insufficiency. Pneumologie 44, 193-194

Laier-Groeneveld G, Schucher B, Criée CP (1997): Die Ursachen der chronischen Hyperkapnie. Med Klin 92, Sondernr. 1, 33-38 
Lassen HCA (1953): A preliminary report on the 1952 epidemic of poliomyelitis in Copenhagen with special reference to the treatment of acute respiratory insufficiency. Lancet $\underline{1}, 37-40$

Laufman H: Planning and building the ICU: problems of design, infection control and cost/benefit. In: Reis MD, Langher D (Hrsg.): The ICU: a cost/benefit analysis. Exerpta Medica, Congress Series, Amsterdam 1986, 709-712

Lawin P (1998): Die geschichtliche Entwicklung der Intensivmedizin in Deutschland, Zeitgenössische Betrachtungen. Anaesthesist 47, 983-992

Léger P, Bedicam JM, Cornette A, Reybet-Degat O, Langevin B, Polu JM, Jeannin L, Robert D (1994): Nasal Intermittent Positive Pressure Ventilation - Longterm Follow-up in Patients with severe Chronic Respiratory Insufficiency. Chest 105, 100-105

Lightowler JV, Wedzicha JA, Elliott MW, Ram FS (2003): Non-invasive positive pressure ventilation to treat respiratory failure resulting from exacerbations of chronic obstructive pulmonary disease: Cochrane systematic review and meta-analysis. BMJ $\underline{326}, 185$

Meduri U, Conoscenti CC, Menashe P, Nair S (1989): Noninvasive Face Mask Ventilation in Patients with Acute Respiratory Failure. Chest $\underline{95}$, 865-870

Nationales Referenzzentrum (NRZ) für Surveillanche von nosokomialen Infektionen (2009): KISS Krankenhaus-Infektions-Surveillance-System. [Online im Internet] URL: http://www.nrz-hygiene.de/surveillance/kiss/

stations-kiss/infektionen/archiv/7e01f82c/ 1098/33/ [Stand: 29.05.2013, $16: 12]$.

Nava S, Rubini F, Zanotti E, Ambrosino N, Bruschi C, Vitacca M, Fracchia C, Rampulla C (1994): Survival and prediction of successful ventilator weaning in COPD patients requiring mechanical ventilation for more than 21 days. Eur Respir J $\underline{7}, 1645-1652$

Nava S, Ambrosino N, Clini E, Prato M, Orlando G, Vitacca M, Brigada P, Fracchia C, Rubini F (1998): Noninvasive Mechanical Ventilation in the Weaning of Patients with Respiratory Failure Due to Chronic Obstructive Pulmonary Disease. Ann Intern Med 128, 721-728

Navab S, Confalonieria M, Rampullab C (1998): Occasional review: Intermediate respiratory intensive care units in Europe: a European perspective. Thorax $\underline{53}, 798-802$ 
Oye RK, Bellamy PE (1991): Patterns of resource consumption in medical intensive care. Chest $\underline{99}$, 685-689

Peter JV, Moran JL, Phillips-Hughes J, Warn D (2002): Noninvasive ventilation in acute respiratory failure - a meta-analysis update. Crit Care Med 30, 555-562

Plötz FB, Slutsky AS, Vught AJ van, Heijnen CJ (2004): Ventilator-induced lung injury and multiple system organ failure: a critical review of facts and hypotheses. Intensive Care Med 으, 1865-1872

Rosenthal GE, Sirio CA, Shepardson LB, Harper DL, Rotondi AJ, Cooper GS (1998): Use of intensive care units for patients with low severity of illness. Arch Intern Med 158, 1144-1151

Safdar N, Dezfulian C, Collard HR, Saint S (2005): Clinical and economic consequences of ventilator-associated pneumonia: a systematic review. Crit Care Med 33, 2184-2193

Scheithauer S, Haefner H, Schwanz T, Schulze-Steinen H, Schiefer J, Koch A, Engels A, Lemmen SW (2009): Compliance with hand hygiene on a surgical, a medical, and a neurological ICU: direct observation versus calculated disinfectant usage. AJIC $\underline{37}, 835-841$

Schönhofer B: Nicht-invasive Beatmung zur Therapie der akuten respiratorischen Insuffizienz. In: Burchardi H, Larsen R, Kuhlen R, Jauch KW, Schölmerich J (Hrsg.): Die Intensivmedizin. 10. Auflage; Springer Medizin Verlag, Heidelberg 2008, 531-543

Schönhofer B, Sortor-Leger S (2002): Equipment needs for noninvasive mechanical ventilation. Eur Respir J 20, $1029-1036$

Schönhofer B, Kuhlen R, Neumann P, Westhoff M, Berndt C, Sitter H (2008): Noninvasive ventilation as treatment for acute respiratory insufficiency. Essentials from the new S3. Anaesthesist $\underline{57}, 1091-1102$

Simonds AK, Elliott MW (1995): Outcome of domiciliary nasal intermittent positive pressure ventilation in restrictive and obstructive disorders. Thorax $\underline{50}, 604-$ 609

Thomas J: Probleme der Beatmung auf Intensivstationen. 4. Erweiterte Auflage; pmi Verlagsgruppe, Frankfurt 1997 
Topel H, Krankenpflegeschule Duisburg e.V. (o. J.): Fortbildung. Basiskurs Pflegefachkraft für außerklinische Beatmung. [Online im Internet:] URL: http://www.evkln.de/fileadmin/ejk/EKN/Krankenpflegeschule/ Basiskurs_Pflegefachkraft_fuer_ausserklinische_Beatmung.pdf [Stand: 09.05.2014, 10:15].

Unertl K, Heininger A (2000): Prävention der nosokomialen Pneumonie. Bundesgesundheitsbl-Gesundheitsforsch-Gesundheitsschutz 43,302-309

Veitshans S: Intermittierende Selbstbeatmung bei akuter Atmungsinsuffizienz und bei Entwöhnung von invasiver Beatmung unter Berücksichtigung des Langzeitüberlebens. Med. Diss. Göttingen 2002

Vincent JL (2003): Nosocomial infections in adult intensive-care units. Lancet $\underline{361}$, 2068-2077

Vincent JL, Bihari DJ, Suter PM, Bruining HA, White J, Nicolas-Chanoin MH, Wolff M, Spencer R, Hemmer M (1995): The prevalence of nosocomial infection in intensive care units in Europe. Results of the European Prevalence of Infection in Intensive Care (EPIC) Study. EPIC International Advisory Committee. JAMA $\underline{274}, 639-644$

Wang F, Wu Y, Bo L, Lou J, Zhu J, Chen F, Li J, Deng X (2011): The Timing of Tracheotomy in Critically ill Patients Undergoing Mechanical Ventilation: A Systematic Review and Meta-analysis of Randomized Controlled Trials. Chest 140, 1456-1465

Winck JC, Azevedo LF, Costa-Pereira A, Antonelli M, Wyatt JC (2006): Efficacy and safety of non-invasive ventilation in the treatment of acute cardiogenic pulmonary edema - a systematic review and meta-analysis. Crit Care 10, R69 


\section{Danksagung}

Herzlich danken möchte ich meinem Doktorvater Herrn Prof. Dr. Gerhard Laier-Groeneveld für die freundliche Überlassung des hochinteressanten Themas meiner Dissertation. Ich bin sehr dankbar für viele anregende Diskussionen und jede erdenkliche Unterstützung seinerseits. Fortwährend und mit großem Zeitaufwand stand er mir warmherzig, geduldig und professionell zur Seite.

Sein kompetenter Rat und sein überaus großes Fachwissen kamen mir während des gesamten Arbeitsprozesses zugute.

Mein besonderer Dank gilt auch Herrn Simon Hanau. Der Atmungstherapeut der Klinik ermöglichte mir tiefgreifende Einblicke in die Praxis der Heimbeatmungs-therapie. Mein universitäres und theoretisches Wissen im Bereich der Pneumologie wurde durch die praktische Anwendung vertieft und erweitert.

Herrn Dr. Thomas Rieger, ärztlicher Direktor und Chefarzt des Instituts für Laboratoriumsmedizin und Klinische Mikrobiologie des Evangelischen Krankenhauses Oberhausen, danke ich herzlichst für die Bereit- und Zusammenstellung der Ergebnisse von mehr als 400 mikrobiologischen Proben.

Bei Diplom-Mathematiker Benjamin Becker, Mitarbeiter des Instituts für Medizinische Statistik der Universitätsmedizin Göttingen, bedanke ich mich für die hilfreichen Tipps bei der Durchführung der statistischen Auswertung. Seine sowohl kritischen als auch äußerst konstruktiven Anmerkungen haben mir beim Durchführen der statistischen Berechnungen sehr geholfen.

Diplom-Psychologin Vanessa Kaes sei herzlich gedankt. Jederzeit war sie geduldig und freundlich bereit, wissenschaftliche Diskussionen zu führen und Ratschläge zu geben. 


\section{Lebenslauf}

Am 03. Januar 1986 wurde ich, Lisa Barbara Hoffmann, als zweites Kind der Eheleute Dr. med. Thomas Hoffmann und Dr. med. Heidi Hoffmann, geb. Koch, in Mainz, geboren.

Von 1992 bis 1996 war ich Grundschülerin der Sonnenschule Attendorn. Daraufhin besuchte ich das Rivius-Gymnasium Attendorn. Dort erlangte ich im Juni 2005 mit den Leistungskursen Erdkunde und Mathematik die allgemeine Hochschulreife.

Von Oktober 2005 bis Juni 2006 besuchte ich das Theologische Seminar Neues Leben.

Im Wintersemester 2006/2007 begann ich das Studium der Humanmedizin an der Heinrich-Heine-Universität Düsseldorf. Im September 2008 legte ich dort den Ersten Abschnitt und im Dezember 2012 den Zweiten Abschnitt der Ärztlichen Prüfung ab.

Seit Juni 2013 arbeite ich nun als Assistenzärztin in der Abteilung für Allgemeinund Viszeralchirurgie des Johanna-Etienne-Krankenhauses in Neuss. 